\title{
Design and Control of Flapping Wing Micro Air Vehicles
}

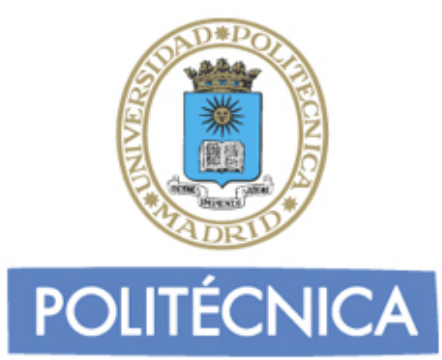

Chao Zhang

Department of Electronics, Informatics and Industrial Engineering

Universidad Politécnica de Madrid, Spain

A thesis submitted for the degree of

Doctor of Philosophy in Automation and Robotics

2016 
Title:

Design and Control of Flapping Wing Micro Air Vehicles

Author:

Chao Zhang

Director:

Prof. Claudio Rossi, Ph.D

Robotics and Cybernetics Group

Tribunal nombrado por el Mgfco. y Excmo. Sr. Rector de la Universidad Politécnica de Madrid, el día de de 2016.

\section{Tribunal}

Presidente:

Vocal:

Vocal:

Vocal:

Secretario:

Suplente:

Suplente:

Realizado el acto de lectura y defensa de la Tesis el día de .......de 2016 .

Calificación de la Tesis:

El presidente:

Los Vocales:

El Secretario: 


\begin{abstract}
Flapping wing micro air vehicles (FWMAVs) have become an important research topic in recent years. Their potential applications, like search, rescue as well as surveillance and reconnaissance, make them attractive solutions with respect to traditional fixed or/and rotatory aerial vehicles. In this thesis, our research work focuses three main aspects of them: flapping transmission mechanism, flight attitude control and morphing-wing structure.

First, we propose a compliant transmission mechanism for a FWMAV, which was inspired by the thorax structures of insects. To better design the driving mechanism, kinematic analysis of the mechanism was derived. In addition, an aerodynamic model of wings was also built to be coupled with the flapping mechanism. Next, we proposed combining two methodologies, virtual-work-based and rigid-body dynamics-based approaches, to calculate and optimize the input torque required from the flapping actuator (DC motor). This allows designing a transmission mechanism that minimizes the sharp shock of the motor. After optimization, the compliant transmission mechanism with well-tuned parameters was shown to reduce the peak input torque up to $66 \%$, compared to a full rigid-body mechanism. Second, we focus on the attitude control of the FWMAV. We proposed a type-2 fuzzy neural network working in parallel with a derivative differential controller to cope with system uncertainties and environmental disturbances. For the fuzzy neural network, two different triangular membership functions, with uncertain width and center, respectively, were used. With respect to these two cases, two new online learning algorithms were employed to update the parameters in both antecedents and consequents of the fuzzy neural network. After that, the stabilities of the two cases were proved by using both Lyapunov and sliding mode control theories. We applied the proposed methods to the attitude control model of the FWMAV. Simulation results demonstrate that the methods could effectively track the desired control signals even with system uncertainties and environmental disturbances.
\end{abstract}


Finally, we explore the effect of a morphing-wing structure inspired by bats. Bats have shown excellent flight characteristics in terms of high maneuverability and good stability by varying the geometry of their wings. A morphing-wing structure that mimics the mechanical system of bats' wings was integrated into the design of a flapping wing robot. To investigate the influence of changing the wing shape on the flight performance of the robot, in terms of lift and thrust forces, several experiments were performed. This approach provides an insight for developing future morphingwing FWMAVs with the purpose of improving their maneuverabilities and flapping efficiency. 


\section{Resumen:}

Los micro vehículos aéreos con aleteo de las alas (FWMAVs por su sigla en Inglés) se han convertido en un tema de investigación importante en los últimos años. Sus aplicaciones potenciales, como búsqueda y rescate, así como vigilancia y reconocimiento, los hacen una solución atractiva con respecto a los vehículos aéreos tradicionales con alas fijas o rotatorias. En esta tesis, nos centramos en tres aspectos principales: mecanismo de aleteo, control de vuelo y estructura de ala con geometría variable.

En primer lugar, se propone un mecanismo de transmisión par un FWMAV, inspirado en la estructura del tórax de los insectos. Para diseñar el mecanismo de accionamiento, se lleva a cabo una análisis cinemático del mismo. Además, se desarrolla un modelo aerodinámico de las alas para ser acoplado con el mecanismo de aleteo. A continuación, se propone la combinación de dos metodologías, basadas en el trabajo virtual y en la dinámica de cuerpos rígidos para calcular y optimizar el par de entrada requerido por el actuador (motor de corriente continua) dedicado al aleteo. Esto permite el diseño de un mecanismo de transmisión que reduce drasticamente los choques del motor. Se demuestra que, después de la optimización, el mecanismo de transmisión con parámetros afinados reduce el pico del par de entrada de hasta un 66,0 \%, en comparación con un mecanismo de transmisión rígido.

En segundo lugar, nos centramos en el control de actitud del FWMAV. Se propone una red neuronal difusa de tipo 2 que trabaja en paralelo con un controlador diferencial derivativo para hacer frente a las incertidumbres del sistema y a las perturbaciones ambientales. Para la red neuronal, se utilizan dos funciones de pertenencia triangulares con anchura y centro diferentes. Se emplean dos nuevos algoritmos de aprendizaje en línea para actualizar los parámetros en los antecedentes y consecuentes de la red neuronal difusa. Seguidamente, la estabilidad de los dos métodos propuestos es probada mediante el uso tanto de la teoría de Lyapunov como del método sliding mode. Los métodos propuestos se aplican al control de actitud del modelo del FWMAV. Los resultado de las simulaciónes demuestran que los controladores desarrollados pueden seguir de forma efectiva las señales de control deseadas, 
incluso con las incertidumbres del sistema y en presencia de perturbaciones ambientales.

Por último, se analiza el efecto del cambio de la geometría de la estructura de las alas inspirado en los murciélagos. Estos animales muestran excelentes caractersticas de vuelo en términos de maniobrabilidad y estabilidad, que obtienen variando la geometría de sus alas. Una estructura de ala con geometría variable que imita el sistema mecánico de alas de murciélago fue integrada en el diseño de un vehículo aéreo con aleteo de las alas. Para investigar la influencia del cambio de la forma del ala en el vuelo del robot, en términos de las fuerzas de elevación y de empuje, se realizaron varios experimentos. Este enfoque proporciona una visión para el desarrollo de futuros FWMAVs con ala mórfica, con el propsito de mejorar su maniobrabilidad y eficiencia en el aleteo. 
To my grandfather, his love always gets me moved and encouraged.

To my parents, their love, wisdom and advising make me go towards my life goal.

To my sisters, their unconditional supports and encourage let me fully concentrate my attention on my study. 


\section{Acknowledgements}

First and foremost, I'd like to express my sincere gratitude to my supervisor, Prof. Claudio Rossi, for his kind guidance and continuous support during my doctoral program. His inspiration, encouragement and advise made my academic journey more wonderful in and beyond the robotics.

I owe special thanks to Associate Prof. Haibao Hu at Northwestern Polytechnical University (NPU), China, for providing me with the facility on the force measurement of our flapping-wing MAV, and Dr. Dong Song from the same university for the kind help and support during the whole process of all the experiments.

I gratefully acknowledge the research fellowship offered by China Scholarship Council (CSC) to perform my doctoral study in Spain. I am also grateful to teachers in the Educational Group of Chinese Embassy in Spain, professors, secretaries, engineers at the Centre for Automation and Robotics (CAR), Universidad Politécnica de Madrid (UPM). Thank all my friends made in Madrid. It is my honour to be friends with you.

Finally, I would like to thank my whole family for their support and encouragement during the period that I pursue my doctoral degree. Their unconditional love always gave me enough confidence and courage to pass through the tough time and complete the study. Words could never express my deepest love and appreciation to them. 


\section{Contents}

List of Figures

List of Tables

1 Introduction 1

1.1 Research Background . . . . . . . . . . . . . . . . 1

1.1.1 Compliant Transmission Mechanisms . . . . . . . . . . . . . . . . 2

1.1.2 Flight Control Strategies . . . . . . . . . . . . . . . . . 2

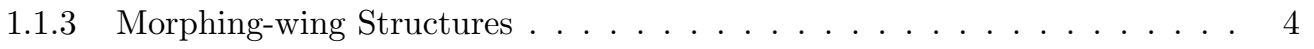

1.2 Research Objectives . . . . . . . . . . . . . . . . . . . . 4

1.3 Thesis Organization . . . . . . . . . . . . . . . . . . 7

2 State-of-the-art Review 9

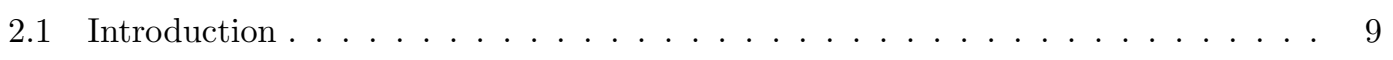

2.2 Compliant Transmission Mechanism . . . . . . . . . . . . . . 9

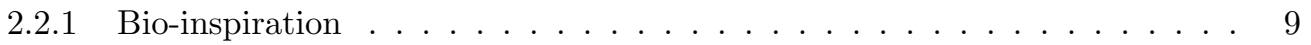

2.2.2 DC motor-based compliant transmission mechanism . . . . . . . . 111

2.2.3 Smart actuator-based compliant transmission mechanism . . . . . . . 16

2.2.3.1 Piezoelectric actuators. . . . . . . . . . . . . . 16

2.2.3.2 Electromagnetic actuators. . . . . . . . . . . . 20

2.2.3.3 Dielectric elastomer actuators. . . . . . . . . . . . 2 21

2.3 Flight Control Strategy $\ldots \ldots \ldots$

2.3 .1 Linear Control . . . . . . . . . . . . . . . . . . . . . . . . 223

2.3 .2 Nonlinear Control . . . . . . . . . . . . . . . . . . . . . . 25

2.4 Morphing-wing Concepts for Flapping wings . . . . . . . . . . . . . . 27

2.4 .1 Bio-inspiration . . . . . . . . . . . . . . . . . . . 27 
2.4.2 Passive Morphing Wings . . . . . . . . . . . . . . . 28

2.4 .3 Active Morphing Wings . . . . . . . . . . . . . . . . . 33

2.5 Conclusion $\ldots \ldots \ldots \ldots \ldots \ldots$

3 Design and Optimization of Compliant Transmission Mechanism 40

3.1 Introduction . . . . . . . . . . . . . . . . . 40

3.2 First-version Compliant Transmission Mechanism . . . . . . . . . . . . . 41

3.2.1 Force Estimation acting on Wing Supporters . . . . . . . . . . . . 44

3.2.2 Optimization Design and Stress Analysis of Wing Supporters . . . . . . 45

3.3 A Second Improved Compliant Transmission Mechanism and Its Kinematics . . . 47

3.3.1 Kinematic Analysis . . . . . . . . . . . . . . . . . . . . . . . . . . 49

3.4 Aerodynamic Torque . . . . . . . . . . . . . . . . . . . . 50

3.5 Input Torque Calculation Based on the Principle of Virtual Work . . . . . . . . 51

3.6 Input Torque Calculation Based on Rigid-body Dynamics . . . . . . . . . . . 53

3.7 Optimization Design . . . . . . . . . . . . . . . . . . . . . . . . . . . 57

3.7 .1 Result Analysis . . . . . . . . . . . . . . . . 5 59

3.8 Conclusions . . . . . . . . . . . . . . . . . . . . . . . . . . . . . . . . . 60

4 Attitude Control and Simulation of a Flapping-wing Micro Air Vehicle 62

4.1 Introduction . . . . . . . . . . . . . . . . . . 62 62

4.2 Dynamic Model of FWMAV . . . . . . . . . . . . . . . . . 63

4.3 Feedback-error-learning Control Frame . . . . . . . . . . . . . . . 65

4.4 Type-2 Fuzzy Neural Network with Triangular Type-2 MFs . . . . . . . . . . . 65

4.4.1 Type-2 Triangular Membership Functions . . . . . . . . . . . . 66 66 . . . . . .

4.4.2 Interval Type-2 A2-C0 Takagi-Sugeno-Kang Model . . . . . . . . . . . 68

4.5 SMC Theory-based Learning Algorithm . . . . . . . . . . . . . . . . . 69

4.5.1 Parameter Update Rules for T2FNN . . . . . . . . . . . . . . . 71

4.5 .2 Stability Analysis . . . . . . . . . . . . . . . 73

4.6 Simulation Results and Analysis . . . . . . . . . . . . . . . 74

4.7 Conclusion . . . . . . . . . . . . . . . . . . . . . . 755 
5 Design and Minor Tests of a Morphing-wing Structure 80

5.1 Introduction . . . . . . . . . . . . . . . . . . . . . . . . . . 80

5.2 Fabrication, Assembly and Actuator . . . . . . . . . . . . . 81

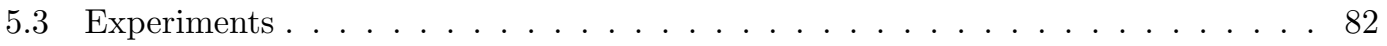

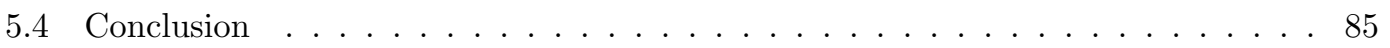

6 Conclusions and Open Challenges

6.1 Conclusions . . . . . . . . . . . . . . . . . . . . . . 86

6.2 Open Challenges . . . . . . . . . . . . . . . . . 87

7 Publications

References

8 Annexes 101

8.1 Proof of Theorem 1 . . . . . . . . . . . . . . . . . . . . . . . . . 101

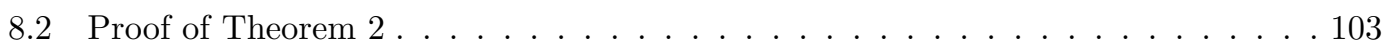

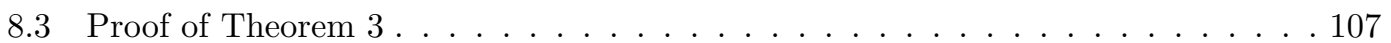




\section{List of Figures}

1.1 Representative models of four primary rigid-body transmission mechanisms. (a) front mounted double pushrods (Chung Hua University, FWMAV (1)). (b) front mounted double crank (Delft University of Technology, Delfly I) (2). (c) front mounted single pushrod (Wright State University, FWMAV (3)). (d) sidemounted crank (Delft University of Technology, Delfly II (2) ) . . . . . . . . . 3

2.1 Diagrammatic cross section of insect thorax mechanisms for flight. Thorax mechanisms with direct flight muscles: (a) upstroke and (b) downstroke. Thorax mechanisms with indirect flight muscles: (c) upstroke and (d) downstroke. Contracting muscles are depicted in red, the tergum is depicted in orange. . . . . . . 11

2.2 DC motor-based compliant transmission mechanisms using linear/torsion springs.

(a) Two ends of a linear spring are connected to the base and the wing leading edge, respectively. Source: (4). (b) A spring is mounted between the rocker of the driving mechanism and its responding wing holder. Source: (5) . . . . . . 12 12

2.3 A 5.8 g flapping mechanism developed at UC Berkeley, in which a linear spring is directly linked the coupler. Source: $(\underline{6})$. . . . . . . . . . . . . 12

2.4 The prototype of a motor-driven flapping-wing micro aerial vehicle. Source: (7, 8) $)$. 13

2.5 Flapping mechanisms used in University of Maryland's Small and Jumbo birds.

(a) The schematic of the compliant flapping-wing mechanism used in Small Bird. Source: (9, 10). (b) The diagram of compliant mechanism for flapping wing actions of Jumbo Bird. Source: (11)) . . . . . . . . . . . . . . . . . . . 13

2.6 A compliant thoracic mechanism at Nanyang Technological University, in which lightweight polyimide films were integrated into the compliant hinges for elastic energy storage. Source: $(12)$. . . . . . . . . . . . . . . . . . . . . . 114 
2.7 The prototype of the Harvard University's FWMAV and its the four-bar transmission mechanism. Note that the rubber flexural hinges are located at joints 3 and 4 while joints 1 and 2 are both rigid joints. Source: $(13,14) \ldots \ldots \ldots$

2.8 Robotic flying insect Lionfly at Pennsylvania State University. (a) Conceptual drawing of the LionFly. (b) A prototype LionFly with zoomed inset showing an end view of the flapping mechanism and actuator. Source: $(15,16) . \ldots . . .18$

2.9 A spherical four-bar-wing system actuated by a bimorph piezoelectric bending actuator. (a) SolidWorks model of a four-bar-wing system. (b) Experimental setup for testing the performance of the spherical four-bar-wing system. Source: (17) . . . . . . . . . . . . . . . . . . . . . . 18

2.10 Harvard RoboBee driven by piezoelectric actuators. Source: $(18,19,20) \ldots$. . . . 19

2.11 A 3.5-cm wingspan, $144 \mathrm{mg}$ weight prototype actuated by an electromagnetic actuator. (a) Top view of the structure. (b) The variation of wing' stroke with the effect of the electromagnetic force. (c) The physical prototype. Source: (21).

2.12 An electromagnetic actuator with flapping frame and experimental test. According to the left exploded view, the actuator consists of six components: (1) Wedge coil, (2) spring magnets, (3) rotor, (4) pivot pins, (5) stopper pin, and (6) frame. Source: $(22,23,24)$. . . . . . . . . . . . . . . . . . . . . . . . . 21

2.13 Integration of a CFRP shell and rolled DEA with a thoracic mechanism to form an insect-inspired wing flapper Source: (25) . . . . . . . . . . . . 2

2.14 (a) Schematic of the relative arrangement of the ten wing bones of a bird. The geometry of the bones is intended to be indicative $(26,27)$. (b) The wing of a lesser dog-faced fruit bat $C$ ynopterus Brachyotis comprises an elastic muscularized membrane that is stretched between the elongated digits of the hand, the hindlimbs, and the body wall. Source: (27) . . . . . . . . . . . . . . . 27

2.15 Three types of passive morphing wing concepts and their responding morphing principles with effects of aerodynamic and inertial loads (AIL). (a) Rigid passive morphing concept with a angular limitation of wings' motion. (b) Flexible Derlin hinge Concept. (c) Compliant carbon fibre (CF) hinge concept. Source: (28). . . 29] 2.16 Starboard passively morphing wing spar. Source: $(29) \ldots \ldots \ldots$ 
2.17 A test ornithopter with passive morphing wings. A compliant spine, which is designed to be stiff during downstroke and flexible during upstoke, is inserted in the leading edge spar of the ornithopter for investigating its influence on the flight performance of the vehicle. Source: $(30,31,32) \ldots \ldots \ldots$. . . . . . . 31

2.18 A passive wing morphing inspired by bird wing morphology. (a) Comparison between the robotic wing and bird wing morphology. Both bird and robotic wings could rotate their wrists(hinge) to sweep forwards (unfold) and backwards (fold). (b) The mechanical sizes of the robotic wing. (c) Wings were constructed with carbon fibre leading edges and spars, Mylar foils and other 3D printed joints. Strings constrain the wing from unfolding until released. (d) Variation of the robotic wing in static configuration. (Top) fully folded, (Bottom) extended and (Middle) partially extended. Source: (33) . . . . . . . . . . . 32

2.19 Bio-inspired wrinkled wings for micro flapping-wing aerial robots. Source: (34). . 33

2.20 Festo SmartBird. . . . . . . . . . . . . . . . . . . . . 34

2.21 The BATMAV actuated by using SMAs wires for flapping and morphing like bats. Source: $(35),(36,37) \ldots \ldots \ldots \ldots$

2.22 Different wing configuration of a robotic batwing flapper with kinematic values closest to median values of model species: $8 \mathrm{~Hz}$ wingbeat frequency, $75^{\circ}$ wingbeat amplitude, $60^{\circ}$ degrees stroke plane angle, 0.45 downstroke ratio, and $40^{\circ}$ wing folding. Front view: (a) Fully depressed. (b) Fully elevated. Ventral view: (c) Fully protracted. (d) Fully retracted. (e) Wing extended. (f) Wing folded.

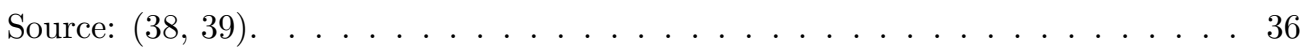

2.23 A bat-like micro aerial vehicle (Batbot) using NiTi shape memory alloy (SMA) artificial muscles to actuate the morphing wing mechanism. Source: (40) . . . . 37

3.1 Detail of the compliant actuation mechanism for a morphing-wing flying robot . 42

3.2 Main body of the morphing-wing flying robot . . . . . . . . . . . 42

3.3 Pseudo-rigid-body model of compliant driving mechanism . . . . . . . . . . 43

3.4 Forces in $x$ and $y$ directions during one circle flapping at joint C. The $x$ and $y$ values of the green points were $0.2859 \mathrm{~N}$ and $-1.3957 \mathrm{~N}$ while those of the magenta ones along $x$ and $y$ axises were $-0.0034 \mathrm{~N}$ and $1.7393 \mathrm{~N}$,respectively. . 44

3.5 Von Mises stress with a horizontal pull force $0.1295 \mathrm{~N}$ and vertical push force $0.69785 \mathrm{~N} \ldots \ldots \ldots \ldots \ldots \ldots \ldots \ldots \ldots$ 
3.6 Von Mises stress with a horizontal pull $0.0017 \mathrm{~N}$ and vertical pull force $0.0125 \mathrm{~N} 47$

3.7 A closeup of the CAD model for a bat-inspired flapping-wing aerial vehicle. . . . 48

3.8 (a) A schematic view of the compliant transmission mechanism during upstroke.

(b) Pseudo-rigid-body model of the compliant transmission mechanism. . . . . . 49

3.9 Variations on the flapping angle of a wing and the torsional angle at compliant joint D during a flapping cycle. . . . . . . . . . . . . . . . 50 50

3.10 The sketch of the velocity analysis on the wing. . . . . . . . . . 52 52

3.11 Variation of the input torque from the motor in function of the torsional stiffness of virtual springs at joint $\mathrm{C}$ and D. Left: $K_{1}=0(\mathrm{RBM}), 0.2,0.4,0.6,0.8$, $1 \mathrm{Nm} / \mathrm{rad}$ while $K_{2}=0 \mathrm{Nm} / \mathrm{rad}$; Right: $K_{2}=0$ (RBM), 0.2, 0.4, 0.6, 0.8, 1 $\mathrm{Nm} / \mathrm{rad}$ whereas $K_{1}=0 \mathrm{Nm} / \mathrm{rad}$. In both cases, $\theta_{3,0}$ and $\theta_{4,0}$ equal $0^{\circ}$ and $90^{\circ}$,

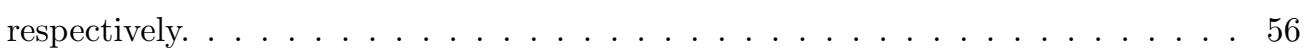

3.12 Variation of the input torque from the motor in function of the neutral angular positions at joint $\mathrm{C}$ and $\mathrm{D}$. :Left: $\mathrm{RBM}, \theta_{3,0}=-13.5^{\circ},-1.5^{\circ}, 10.5^{\circ}, 22.5^{\circ}$, $33.9^{\circ}$ while $\theta_{4,0}=90^{\circ}$; Right: RBM, $\theta_{4,0}=90^{\circ}, 91.5^{\circ}, 93^{\circ}, 94.5^{\circ}, 96.5^{\circ}$ while $\theta_{3,0}=0^{\circ}$. In both cases, $K_{1}$ and $K_{2}$ equal 0.4 and $0.6 \mathrm{Nm} / \mathrm{rad}$, respectively. . . 57

3.13 Input torque required of the flapping mechanism with three different transmission systems: Rigid-body Mechanism (RBM); Compliant Transmission Mechanism with constraints Case I (CTM I); Compliant Transmission Mechanism with constraints Case II (CTM II) . . . . . . . . . . . . . . 60 60

4.1 Feedback-error-learning control frame incorporating a T2FNN structure. . . . . 66

4.2 Triangular type-2 fuzzy set with uncertain width (a) and uncertain center (b). . 67

4.3 The structure of Interval type-2 A2-C0 TSK FNN using triangular MFs with uncertain width $(\mathrm{a})$ and uncertain center $(\mathrm{b}) . \ldots \ldots$. . . . . . . . . 70

4.4 Attitude control responses of a FWMAV in which a T2FNN using triangular MFs with uncertain width (UW) when tracking the sinusoidal reference signals.

(a) Pitch response. (b) Yaw response. (c) Roll response. . . . . . . . . . . . . . . 76

4.5 Attitude control responses of a FWMAV in which a T2FNN using triangular MFs with uncertain center (UC) when tracking the sinusoidal reference signals.

(a) Pitch response. (b) Yaw response. (c) Roll response. . . . . . . . . . . . . . . 77 
4.6 Attitude control responses of a FWMAV in which a T2FNN using triangular MFs with uncertain width (UW) when tracking the step reference signals. (a) Pitch response. (b) Yaw response. (c) Roll response. . . . . . . . . . . . . . . . 78

4.7 Attitude control responses of a FWMAV in which a T2FNN using triangular MFs with uncertain center (UC) when tracking the step reference signals. (a) Pitch response. (b) Yaw response. (c) Roll response. . . . . . . . . . . . . . . . 79

5.1 The whole body of a flying robot $\ldots \ldots \ldots \ldots$

5.2 Force test platform of the flying robot $\ldots \ldots \ldots$. . . . . . . . . . 83

5.3 Lift forces generated by full wings at $\mathrm{AoA}=23^{\circ} \ldots \ldots \ldots$

5.4 Thrust forces generated by full wings at $\mathrm{AoA}=23^{\circ} \ldots \ldots \ldots$

5.5 Roll moment generated by single morphing wing at $\mathrm{AoA}=13^{\circ} \ldots \ldots$. . . . . . 84

5.6 Roll moment generated by a single morphing wing at $\mathrm{AoA}=18^{\circ} \ldots \ldots$. . . . 84

5.7 Roll moment generated by a single morphing wing at $\mathrm{AoA}=23^{\circ} \ldots \ldots \ldots$ 


\section{List of Tables}

2.1 Comparison among various compliant flapping transmission mechanisms driven by DC motors. . . . . . . . . . . . . . . . . . 15

2.2 Overview characteristics of smart actuators. Source: (41). . . . . . . . . . . . . 17

2.3 Comparison among various compliant flapping transmission mechanisms driven by smart actuators. . . . . . . . . . . . . . . . . . . 23

2.4 Comparison among various bio-inspired morphing concepts for flapping-wing aerial ve-

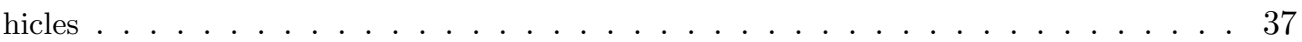

3.1 Key Dimensions of Compliant Actuator Mechanism . . . . . . . . . . . . . 43

3.2 Input Parameters for the Cross-section Optimization of wing supporters . . . . . 46

3.3 Component Dimensions of the Compliant Transmission Mechanism (see also Fig.

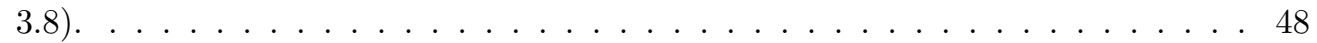

3.4 Parameters used for design optimisation . . . . . . . . . . . . . . . . . 59

3.5 Optimisation results for two kinds of constraint conditions . . . . . . . . . . . . 60

5.1 Sizes of Wing Bone Components . . . . . . . . . . . . . . . . . . . 81

5.2 Key parameters of the morphing-wing flying robot . . . . . . . . . . . . 82 


\section{1}

\section{Introduction}

\subsection{Research Background}

Flapping-wing micro air vehicles (FWMAVs) are a class of unmanned aircraft which imitate flight characteristics of natural organisms, e.g., birds, bats, and insects. FWMAVs mainly contain two kinds of aerial vehicles: one is a bird-size flapping-wing aerial vehicle which is generally called "ornithopter". The other is a much smaller flapping-wing aerial vehicle which generally mimics flapping wing motions of insects or very small birds (e.g. hummingbirds) (42). In 2005, the US DARPA even extends the size of an aerial vehicle into nano scale. A nano air vehicle (NAV) is defined to be no larger than $7.5 \mathrm{~cm}$ or heavier than $10 \mathrm{~g}$ (carrying a $2 \mathrm{~g}$ payload). Here, no matter what kinds of the aerial vehicles the aircraft belong to, they are all regarded as FWMAVs.

FWMAVs normally have an ultra-light weight with compact electronics and surveillance detection equipment. Their flights produce much less noise comparing with fixed and/or rotatorywing aerial vehicles due to their lower work frequency. Thus, their quiet flights accompanying miniature physical sizes make them difficult to detect, easy to quickly deploy, and relatively inexpensive to fabricate (42). These features make them suitable for many potential applications, from search and rescue in buildings or under collapsed structures, sensing of chemical leaks in industry, detection of radiations in nuclear plants as well as surveillance and reconnaissance (43).

The main components of FWMAVs usually consist of a power source, actuators, control units, a flapping transmission mechanism, wings, and essential supporting structures (44). This thesis focusses on optimization design of the flapping transmission mechanism, modelling and 
control of attitude, and the fabrication and tests of a prototype with morphing wings.

\subsubsection{Compliant Transmission Mechanisms}

Currently, most flapping-wing aerial vehicles use rigid-body mechanisms as their transmission systems due to the high forces transmitted and easier controllability. Gerdes et al. (2) categorized the flapping mechanisms used in bird-size FWMAVs into four classifications, i.e., (1) front mounted double pushrods; (2) front mounted double cranks; (3) front mounted single pushrod; and (4) side-mounted crank (2). Representative designs of the four kinds mechanisms are shown in Fig. 1.1. As for the insect-size FWMAVs, like Nano Hummingbird (45), they usually don't have tail wings and only use a couple of wings with large flapping amplitudes and high flapping frequency to control their attitudes, i.e., pitch, yaw and roll. In this case, much more complicated flapping driving mechanisms are required and furthermore must be elaborately designed in their narrow bodies. Recently, some interesting attempts of transmission mechanisms, such as dual series four-bar linkage and string-based designs (45), six-bar linkage design (46), a rack-pinion mechanism (47) and a Watt straight-line linkage system (48), appear to apply to FWMAVs for flight.

Unlike rigid-body mechanisms, compliant mechanisms generally contain flexural joints, segments or other elastic components which can transform motions, forces and energies through deformations of the compliant elements. Compared to rigid-body mechanisms, compliant mechanisms have several benefits, such as reduced wear, no friction and backlash and compatibility with micro-electro-mechanical system (MEMS) processing techniques (15, 49). On the other hand, compliant mechanisms have to face a fatigue problem of flexible components. However, much progresses that have been made in theoretical research (like pseudo-rigid-body theory(49)) and advanced fabrication techniques (like laser micromachining(50), surface micromachining (51), and polymer micromachining (52)) that reduce such problem and speed up practical applications of MAVs.

\subsubsection{Flight Control Strategies}

The flight dynamics of flapping MAVs are complex since they are non-linear time periodic systems (53). At present, most research on dynamic modelling of insect-scale or bird-scale FWMAVs are carried out by using first-principles modelling (54). In (55, 56), Deng et al integrated 6-DoF (degrees of freedom) Newton-Euler equations, quasi-steady flapping aerodynamics, and linear actuator dynamics into a non-linear model for an insect-scale FWMAV. Grauer et al 

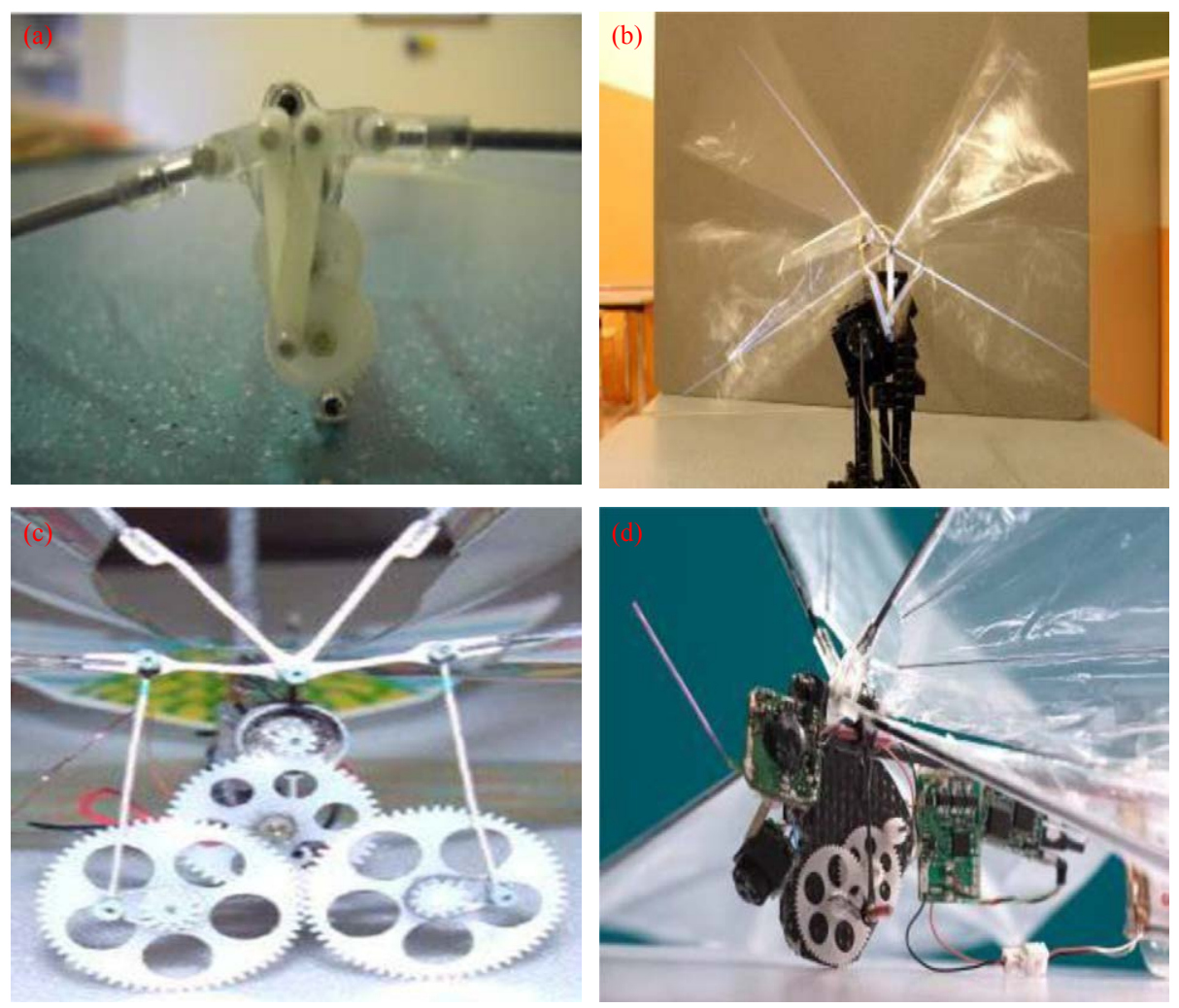

Figure 1.1: Representative models of four primary rigid-body transmission mechanisms. (a) front mounted double pushrods (Chung Hua University, FWMAV (1)). (b) front mounted double crank (Delft University of Technology, Delfly I) (2). (c) front mounted single pushrod (Wright State University, FWMAV (3)). (d) side-mounted crank (Delft University of Technology, Delfly II (2)).

(57) combined Lagrange equations with averaged wing-flapping aerodynamics together for a bird-scale robot. In (58), Colorado et al. proposed a rigid-body dynamic model to estimate the overall energy cost of the flapping and morphing-wing motions and their impacts onto center of mass (CoM) of the robot BaTboT. Currently, the most widely used controller strategies for the dynamics of FWMAVs are proportional-integral-derivative (PID) controllers, which have been successfully adopted on FWMAVs, e.g., Delfly II (59) and the FWMAV of University of California, Berkeley (60). Researchers at Harvard Microrobotics Laboratory recently applied proportional control (61), adaptive control (62), and model-free control (63) to their nano-scale RoboBee to achieve fundamental autonomous flight. Lampton et al (64, 65) suggested that due to the unsteady aerodynamics in complex environments, a neural-network or 
learning-type control concept would have more advantages in adaptability and robustness than traditional control methodologies. Thus, in this thesis, we propose an approach based on fuzzy neural networks to control the attitude and make a comparison with the conventional method (proportional-derivative (PD) control).

\subsubsection{Morphing-wing Structures}

The morphing wing concept (i.e., wings with variable geometry) has interested researchers for many years. At the beginning, the majority of wing shape modification techniques appear in military aircraft, e.g., X-5, F-111, Su-24, F-14 and B-1 (66). Recent collaborative projects on morphing aircraft can refer the Mission Adaptive Wing (MAW) program (67), Aircraft Morphing program (68), DARPA Morphing Aircraft Structures (MAS) program (69). Later, bio-inspired morphing wings concepts emerge in unmanned aerial vehicles (UAVs). In UAVs, the geometry variation is attractive due to its relatively short development times, easy modifications and maintenance (70), and dramatically reduced certification and qualification issues (66).

Only in recent years, integrating morphing capabilities into FWMAVs has emerged as an alternative for traditional FWMAVs, generally provided with rigid and non-variable wings. The capability to morph the wing shape or configuration during flight has been demonstrated to be an effective way to improve the adaptability to multiple flight conditions (71, 72, 73). Besides that, reconfiguring the wing shape can also enhance the maneuverability and meanwhile reduce energy consumption during flight (74). Furthermore, recent advances in smart materials actuators technologies allow further development of bio-inspired morphing wings, since they can dramatically reduce weight, significantly reduce the control complexity, improve the reliability and lower the production cost (66). Also, the possibility of using new materials (e.g., shape memory alloys) that can be used as both actuators and sensors within the structure is a clear advantage (75)

Morphing-wing research on flapping-wing micro air vehicles is still relatively young. In this thesis, we introduce a morphing-wing structure inspired by bats into the fabrication of wings and explore its influences on the maneuverability as well as lift and thrust forces.

\subsection{Research Objectives}

In this thesis, we tackle the problems stated in the previous section. 
- Compliant Flapping Mechanism.

In the literature, the amount of research on the compliant flapping mechanism in the field FWMAV is relatively small. Moreover, most papers focus on manufacture procedures and processes, and only very few of them mention the details of optimization design. In this thesis, we proposed two approaches to optimize the design of the compliant transmission mechanism for flapping. In detail, a compliant transmission mechanism with elastic energy-storing components was proposed at first, which is inspired by the thorax structure of insects. To better design the driving mechanism, kinematic analysis of the structure was carried out. In addition, an aerodynamic model of wings was also built to couple with the mechanism. For realizing the optimal design of this transmission mechanism and thereby reducing the sharp shock of the motor, we put forward two methodologies to optimise primary attributes at compliant joints in the mechanism. One is based on the principle of the virtual work while the other is on the basis of rigid-body dynamics. The first can directly calculate the total virtual work of the whole mechanism including the virtual work of aerodynamic forces. Then, according to the principle of virtual work, the torque required to keep the stable flight can be derived. An optimization algorithm is then used to search the optimal parameters satisfying the design requirements. As for the dynamics-based method, it is necessary to calculate kinetic and potential energies of every element. Then, we use the Lagrange's equation to calculate the torque needed to drive the transmission mechanism while considering the influence of the aerodynamic forces into the Lagrange's equation. Similarly, the last step is to find the optimal parameters for the design as before.

Compared to other optimization methods, our methods are suitable for pseudo-rigid models. Furthermore, the elastic potential energy stored in the compliant joints can be easily taken into account. The proposed methods can be generalized to design and optimize other compliant transmission mechanisms.

\section{- Flight Control Strategy.}

In the literature, both linear control and nonlinear control strategies for fight can be found. For linear control methods, dynamic models are usually used, which almost derive from the simplification and approximation of six-DoF flight models for ordinary standard aircraft. These simplification and approximation get linear controllers hardly deal 
with system uncertainties and extra disturbances. Nonlinear controller have better performance in this regard. However, considering variable unsteady aerodynamic fluids and environmental disturbances, general nonlinear controller may not be qualified. Thus, neural-network or learning-type controllers have been proposed. Type-1 fuzzy neural networks have been investigated for control schemes for flapping wing micro air vehicles. In this thesis, we would extend the type- 1 fuzzy neural networks into type-2 fuzzy neural networks, which have been demonstrated to have much stronger capabilities in coping with uncertainties than type-1 counterparts. Moreover, a feedback-error-learning control frame was employed, in which a type-2 fuzzy neural network works in parallel with an ordinary proportional derivative controller.

Meanwhile, to verify the reliability of the method, two different triangular membership functions were embedded into the type-2 fuzzy neural network, respectively. With respect to the two cases, we proposed two online learning algorithms to update the parameters in both antecedents and consequents of the fuzzy neural network, for improving the robustness of the system and minimizing the disturbance of the environment. Based on the sliding mode control theory, the stabilities of the two cases were proved by using the Lyapunov theory. Finally, we applied the proposed methods to the previous dynamic model and control the attitude of the FWMAV.

\section{- Morphing-wing Structure.}

Research on variable geometry wings has mainly focussed on passive morphing-wing structures, capable of changing the compliance of leading edge spars for wings. These have to be set at the primary design phase and are therefore not controllable. Here, we will deal with active, controlled morphing-wings. In this thesis, new improvements on the morphing-wing structures are made on the basis of the work in (40, 58).

We aim to explore the effects of a morphing-wing structure inspired by bats. Bats have shown excellent flight characteristics in terms of high maneuverability and good stability by varying the morphological shapes of wings. A morphing-wing structure that mimic the mechanical system of bats' wings was integrated into the design of our flapping-wing robot. To explore the influences of changing the wing shape on the flight performance of the robot, in terms of lift and thrust forces, several experiments were performed. In this research, a few number of parameters are changed: the surface of wing shape and the pitching amplitude which determines the angle of attack. Although the number of 
parameters is limited, the study of these parameters will provide us great insights in flight performance of a morphing-wing MAV.

\subsection{Thesis Organization}

This thesis consists of six chapters. The first and last chapters are introduction and conclusion parts, respectively. Each of the four chapters in the middle begins with a brief introduction, which points out a problem to be solved and the methods adopted. At the end of every chapter, a short summary with brief remarks on the topic is presented. In detail, the main chapters are organized as follows:

In Chapter 2, we introduce the state-of-the-art review of flapping wing micro air vehicles. This review mainly focuses on three aspects, i.e., compliant transmission mechanisms, flight control strategies and morphing-wing concepts. Compliant transmission mechanism can be divided into two categories on the basis of the types of actuators, DC motor-based and smart actuators-based compliant transmission mechanisms. The control strategies can be reviewed from linear and non-linear two aspects. As for the morphing-wing concepts, active and passive morphing-wing structures are depicted.

In Chapter 3, we propose a compliant transmission mechanism learning from insects. Its purpose is of minimizing the peak input torque required from a driving motor. In order to maintain the stability of flight, minimizing the peak input torque is necessary. To this purpose, we put forward two modelling methods on the compliant transmission mechanism. One is virtual-work-based while the other is based on rigid-body dynamics. Finally, the mechanism was optimized in terms of of primary attributes of compliant joints (i.e., the torsional stiffness of virtual spring and the initial neutral angular position).

Chapter 4 focuses on the attitude control of flight based on a type-2 fuzzy neural network. In order to improve the adaptability of the approach, a sliding mode control theory-based learning algorithm was also taken into consideration and the stability analysis of the control strategy was proved. Finally, we applied the adaptive fuzzy neural method to the attitude control of the FWMAV. Simulation results showed that the proposed method could fast and stably follow the desired attitude trajectories without steady state errors, compared to a conventional proportional derivative controller used alone.

In Chapter 5, we attempt to find the effect of the morphing-wing structure on the maneuverability as well as lift and thrust forces. In this part, we briefly introduced the bio-inspiration, 
and the fabrication process of morphing-wing structure. After that, several experimental tests were carried out. 


\section{State-of-the-art Review}

\section{$2.1 \quad$ Introduction}

Research on aircrafts that imitate flying mechanisms from biological organisms (e.g. birds, bats, and insects) to achieve lift and thrust has been ever increasing in the last years. During this period, a variety of hobbyists, research groups and robot companies have devoted their efforts to develop biomimetic flying vehicles. The research topics presented in this chapter are related to flapping-wing micro vehicles (FWMAVs). We review the state-of-the-art research of FWMAVs in three aspects:

- Compliant Transmission mechanism.

- Flight Control Strategy.

- Morphing-wing Structure.

We hope, this review could providing readers a general perspective of currently existing research trends on FWMAVs, and meanwhile allow them to understand our starting points on the research described in this thesis.

\subsection{Compliant Transmission Mechanism}

\subsubsection{Bio-inspiration}

The capability to store and release elastic energy is an important characteristic of most animals' locomotion systems, for flight, in legged locomotion, as well as in undulatory fish swimming. Here, we focus on mechanisms for storing and releasing elastic energy at high rates for flappingwing flight. During flapping, as a wing approaches the end of each stroke, the kinetic energy 
of the wing is stored in the musculoskeletal system, decelerating the wings toward the end of a wing stroke and minimizing the sharp shock of the beating wing. Afterwards, the stored elastic energy is released, helping accelerating the wing. The kinematic energy is stored mainly in two different ways: in the flight muscle system $(76,77)$ and in the elasticity of the thoracic skeleton (including the cuticle and wing hinges) (78).

This thesis is centred on the second strategy. As Wootton (79) pointed out, the insect thorax is an interesting system of light, thin composite shells and plates, which is interesting for FWMAVs due to the minimal inertial of its moving parts. Springy, resilient structures, which deform elastically and are capable of elastic energy storage, are also beneficial for an oscillating system. Fair insensitivity to scaling effects is another advantage of insects' thoraxes.

Therefore, to design a small, effective and versatile flapping mechanism, engineers have centered their attention to the thorax of the insects. The insects' thorax can be described as a box with the sides (pleura) and base (sternum) rigidly fused, and the wings attached to the pleura by flexible membranes (80). To power their flight, a series of muscles are involved. These muscles can be classified into two types: direct flight muscles and indirect flight muscles. The direct flight muscles, including the basalar, subalar, and third axillary muscles, insert directly on the wing hinge sclerites or on axillary sclerites or movable sclerites of the pleuron of the thorax. The indirect flight muscles power wing movements by changing the shape of the elastic thorax. These are power muscles and include the dorsal longitudinal muscles that arch the tergum and the dorsoventral and oblique dorsal muscles (81).

As shown in Fig 2.1(a), the upward stroke is produced by contracting the muscles (in red) that connect to wings inside the pivotal point. In Fig. 2.1(b), the muscles that attach to wing bases outside the pivot point contract, causing downward wing stroke. The more advanced insects, like Diptera, use both direct and indirect muscles for flight: direct muscles control wing orientation while indirect muscles provide flapping motion. As shown in Fig. 2.1.(c), muscle contraction causes the tergum, together with the base of the wing, to be pulled down and thereby to force the wings upward. The downward flap is produced by the contraction of the second set of muscles, which run from front to back of the thorax, thereby slightly lifting the tergum and the attachment base of the wings and finally moving the wings downward (Fig. 2.1(d)). At each cycle, the energy is stored through the elastic deformation of thorax in shape when flight muscles relax. 

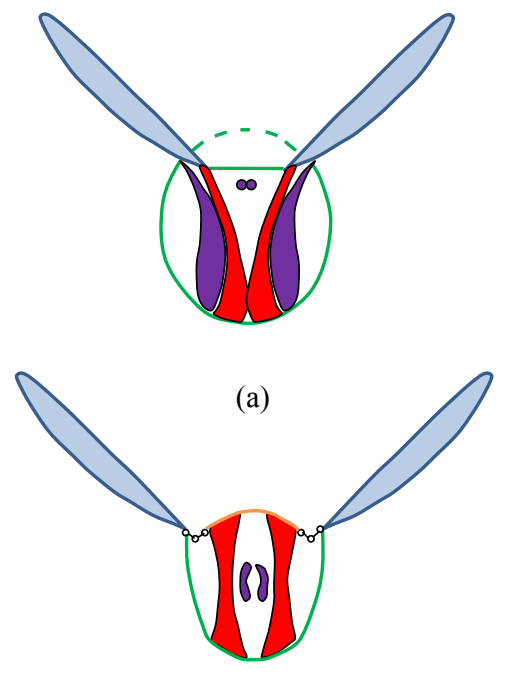

(c)

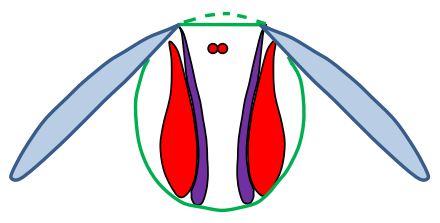

(b)

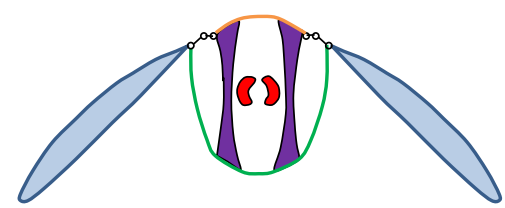

(d)

Figure 2.1: Diagrammatic cross section of insect thorax mechanisms for flight. Thorax mechanisms with direct flight muscles: (a) upstroke and (b) downstroke. Thorax mechanisms with indirect flight muscles: (c) upstroke and (d) downstroke. Contracting muscles are depicted in red, the tergum is depicted in orange.

According to the type of actuators used in current FWMAVs, compliant transmission mechanisms (CTMs) can be divided into two major categories: DC motor-based and smart actuatorbased CTMs. Next, the DC motor-based CTMs will be described firstly in the following.

\subsubsection{DC motor-based compliant transmission mechanism}

DC motors are the most popular actuators for FWMAVs because they are reliable, versatile, cheap and easily available in the market (2).

S.K. Agrawal at the University of Delaware has done an intensive research on the design, kinematic and dynamic analysis, and simulation of FWMAVs that are actuated using DC motors especially regarding the design of the driving system for flapping flight (4, 5, 82, 83). Even though most of their work concentrates on the study of rigid-body transmission systems, attempts that integrate compliant elements into flapping mechanisms have been proposed. In (4), it presented an energy storage mechanism (shown in Fig. [2.2(a)) where linear springs act as elastic energy storage units. When a wing approaches to stop at the end of an upstroke, the two linear springs are fully stretched and the kinetic energy of wings is transformed into elastic potential energy of the spring. During the subsequent downstroke, the potential energy 


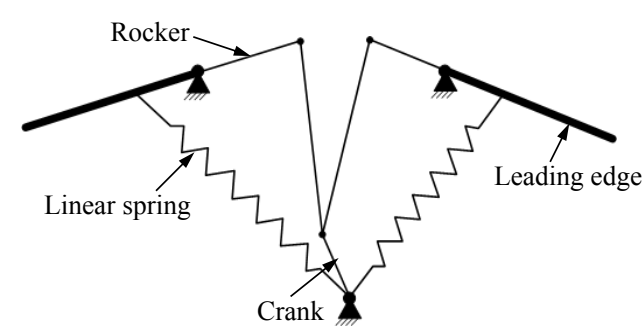

(a)

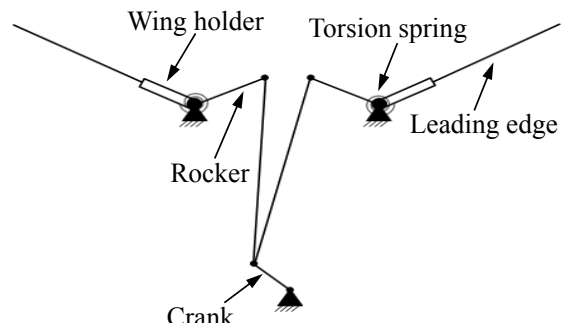

(b)

Figure 2.2: DC motor-based compliant transmission mechanisms using linear/torsion springs. (a) Two ends of a linear spring are connected to the base and the wing leading edge, respectively. Source: (4). (b) A spring is mounted between the rocker of the driving mechanism and its responding wing holder. Source: (5).

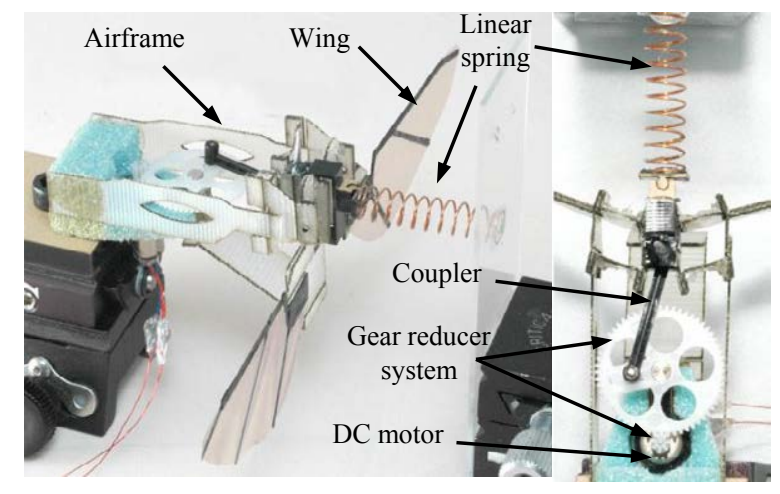

Figure 2.3: A $5.8 \mathrm{~g}$ flapping mechanism developed at UC Berkeley, in which a linear spring is directly linked the coupler. Source: (므).

is released. A similar idea appears in (5), with a different mounting location of the springs (see Fig. 2.2(b)). In the first figure, the two ends of a linear spring are connected to the base and a wing leading edge in the same side, whereas the second uses a torsion spring to bridge a rocker and the responding wing holder. In addition, Baek et al. (6) used a linear spring to directly connect the coupler of the four-bar transmission mechanism and the body frame, as shown in Fig. 2.3. According to their experiments, by integrating a linear spring into the flapping mechanism, the average power could be reduced by $30 \%$.

Recently, coil springs have also been directly coupled with DC motors for directly driving flapping wings toward resonance. Campolo et al. (7, 8, 84) presented a proof-of-concept flapping-wing micro aerial vehicle shown in Fig. 2.4. Their prototype consists of two brushed DC motors, two 7-cm-length wings, two helical springs, and two shaft-spring-wing couplers. The pair of small helical springs is treated as the compliant structures for energy storage and 


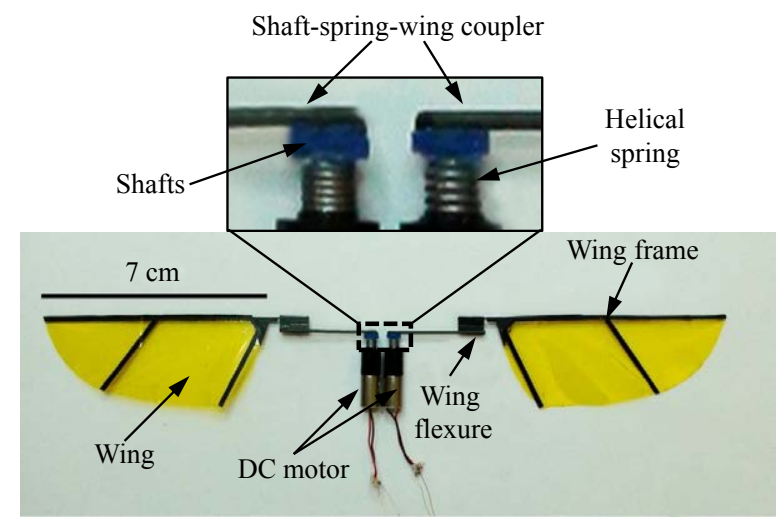

Figure 2.4: The prototype of a motor-driven flapping-wing micro aerial vehicle. Source: $(7,8)$ ).

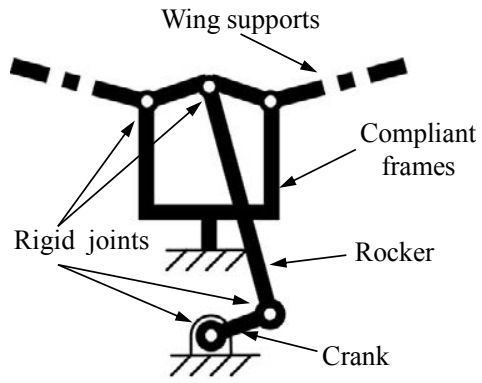

(a)

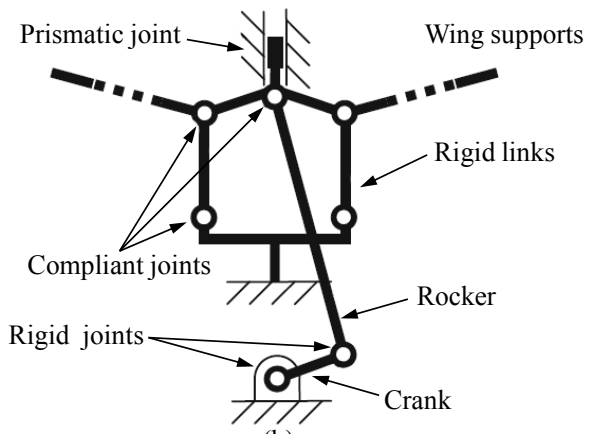

(b)

Figure 2.5: Flapping mechanisms used in University of Maryland's Small and Jumbo birds. (a) The schematic of the compliant flapping-wing mechanism used in Small Bird. Source: (9, 10). (b) The diagram of compliant mechanism for flapping wing actions of Jumbo Bird. Source: (11)).

recovery. The two separate DC motors can drive the individual wing to resonate, respectively. Experiments demonstrated the prototype can successfully lift off and the maximum lift-toweight ratio can be achieved at the flapping frequency $10 \mathrm{~Hz}$. A similar principle is presented in $(85,86)$.

The described FWMAVs could lift off and successfully fly, but face a major drawback: the additional springs do not reduce joint friction energy losses (12), and also add weight. Therefore, structures that combine functions of mechanical parts with elastic energy-stored components (87) seem to be more promising.

The University of Maryland's Small Bird (9, 10) demonstrates a good application of compliant mechanisms in the FWMAV. Figure 2.5(a) shows the schematic concept of the compliant mechanism for flapping motions. The compliant transmission frame was manufactured as a 


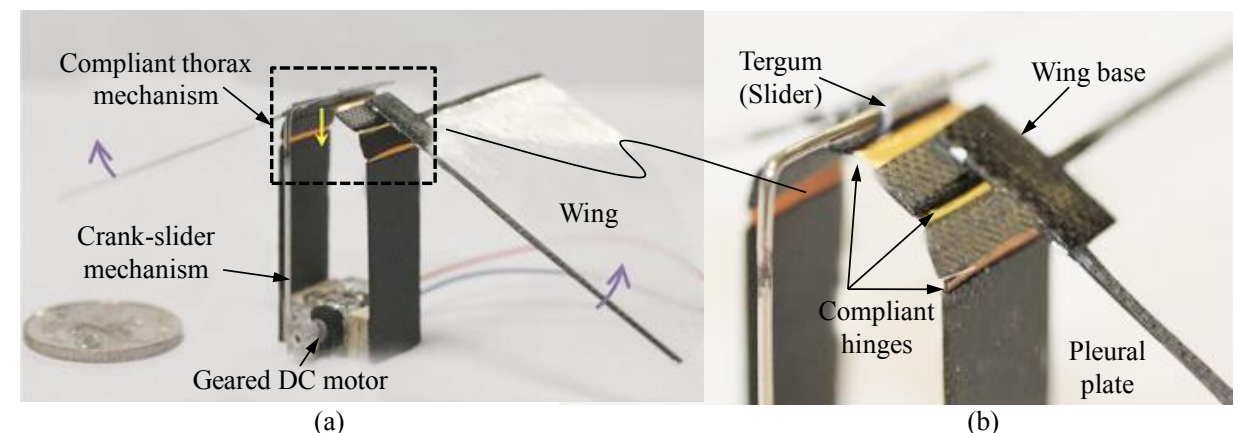

(a)

(b)

Figure 2.6: A compliant thoracic mechanism at Nanyang Technological University, in which lightweight polyimide films were integrated into the compliant hinges for elastic energy storage. Source: (12).

single piece by using injection moulding. This mechanism reduces the use of traditional joints, lowers the weight of the transmission, decreases the part count, and improves overall efficiency of the transmission. A multi-material compliant mechanism was also developed as driving mechanism for a larger Jumbo Bird (11). The mechanism is shown in Fig. 2.5(b). The miniature hinges were adopted to carry out selective and localized compliance in the multi-material structure, which has been demonstrated to be efficient and feasible for successful flight tests.

Lau et al. (12, 88) proposed a similar compliant mechanism, which is shown in Fig. 2.6. Lightweight polyimide film hinges, which were chosen as elastic elements to store inertial energy, were integrated into the mechanism. In order to strengthen the stiffness of polyimide film hinges, carbon-fibre reinforced polymer (CFRP) plates were also used in order to build the system. Due to non-linear stiffness characteristic of compliant hinges, the mechanism can abruptly slow down the wings toward the end stroke and subsequently accelerate to reverse the wings. Their research results demonstrated that the compliant transmission mechanism could save almost $21 \%$ power needs when flapping at $25 \mathrm{~Hz}$, compared to a rigid-body mechanism. Furthermore, using the compliant mechanism has led to needing less power to produce the same thrust needed for a full rigid-body mechanism.

Similarly, Sahai et al. (13, 14) attempted to integrate flexural hinges into a four-bar compliant flapping transmission for a FWMAV with approximately 3 grams of weight. A distinguishing feature of the mechanism is using rubber-based flexures in two of its joints (joints 3 and 4 , see Fig. 2.7). According to their experiments, not only did compliant mechanism save up to $20 \%$ of the input power and $1 \%$ of the weight, but also produced more thrust. 


\subsection{Compliant Transmission Mechanism}

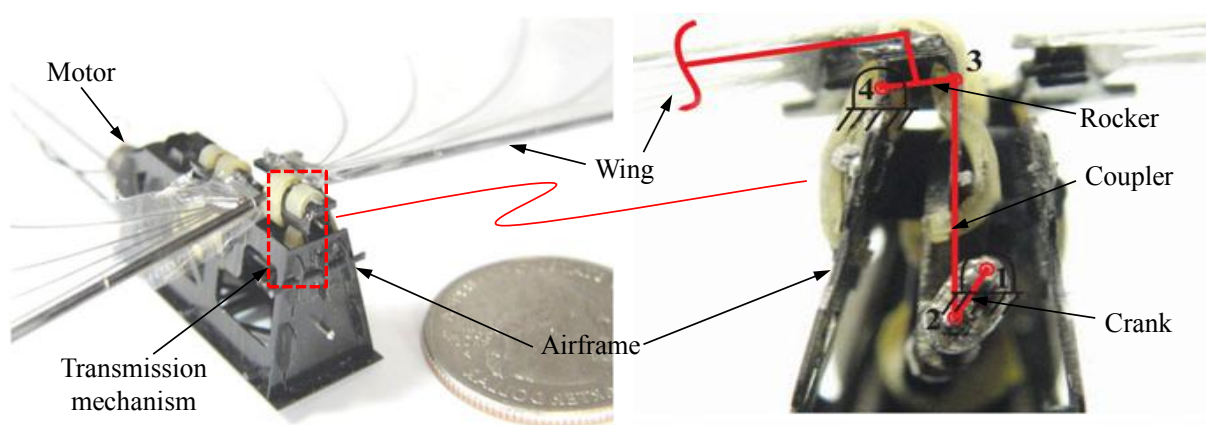

Figure 2.7: The prototype of the Harvard University's FWMAV and its the four-bar transmission mechanism. Note that the rubber flexural hinges are located at joints 3 and 4 while joints 1 and 2 are both rigid joints. Source: (13, 14).

Table 2.1: Comparison among various compliant flapping transmission mechanisms driven by DC motors.

\begin{tabular}{|c|c|c|c|c|c|c|c|}
\hline Group & $\begin{array}{l}\text { Wing } \\
\text { Span } \\
(\mathrm{cm})\end{array}$ & $\begin{array}{c}\text { FWMAV } \\
\text { Weight } \\
(\mathrm{g})\end{array}$ & $\begin{array}{c}\text { Compliant } \\
\text { Element }\end{array}$ & $\begin{array}{l}\text { Weight of } \\
\text { Compliant } \\
\text { Element(g) }\end{array}$ & $\begin{array}{c}\text { Compliant } \\
\text { Stiffness }\end{array}$ & $\begin{array}{c}\text { Compliant } \\
\text { Mass Ratio } \\
(\%)\end{array}$ & $\begin{array}{c}\text { Maximum } \\
\text { Power } \\
\text { Saving }\end{array}$ \\
\hline \multicolumn{8}{|l|}{ Univ. of Delaware } \\
\hline FWMAV (4) & 69 & 46 & Linear Spring & - & $375.2^{\mathrm{a}}$ & - & $56.3 \%$ (in torque) \\
\hline \multicolumn{8}{|l|}{ Univ. of California, Berkeley } \\
\hline FWMAV $(\underline{6}$ & $8^{\mathrm{c}}$ & 5.8 & Linear Spring & $0.259^{\mathrm{c}}$ & $140^{\mathrm{a}}$ & 4.5 & $30 \%$ \\
\hline \multicolumn{8}{|l|}{ Carnegie Mellon Univ. } \\
\hline FWMAV 8 & 14 & 2.7 & Coil Spring & $0.12^{\mathrm{c}}$ & $2.83^{\mathrm{b}}$ & 4.4 & - \\
\hline Uni. of Maryland & & & Compliant & & & & \\
\hline Small Bird(9 10) & 33 & 12.8 & Segments & $0.01^{\mathrm{d}}$ & $0.034^{\mathrm{b}}$ & 0.08 & - \\
\hline Univ. of Maryland & & & Compliant & & & & \\
\hline Jumbo Bird(11) & 63.5 & 72.5 & Joints & - & - & - & - \\
\hline Harvard Univ. & & & Rubber & & & & \\
\hline FWMAV (13, 14) & 12.4 & 3 & Strips & 0.02 & $3.2^{\mathrm{b}}$ & 0.6 & $20 \%$ \\
\hline Nanyang Technological Univ. & & & Polyimide Film & & & & \\
\hline FWMAV $(\overline{12})$ & 10 & 3.51 & Strips & 0.066 & $0.14 \sim 4.3^{\mathrm{b}}$ & 1.9 & $31 \%$ \\
\hline
\end{tabular}

a $\mathrm{N} / \mathrm{m}$.

b $\mathrm{mNm} / \mathrm{rad}$.

c For detail information, please refer to Ref.(12).

d Estimated for four 17.5-mm-length, 0.89-mm-width and 1.52-mm-depth compliant segments, made of ABS material. Such material has a Young modulus of $2.3 \mathrm{GPa}$, and a density $1040 \mathrm{~kg} / \mathrm{m}^{3}$.

Table 2.1 shows a comparison among various DC motor-based FWMAVs that adopted compliant elements into their drive mechanisms. It can be seen that the weight ratios of compliant elements are far smaller than the counterparts of springs. Moreover, the use of compliant mechanisms for driving FWMAVs can save input energies for flight, which to some 
extent can improve the flight duration and/or available payload of FWMAVs.

\subsubsection{Smart actuator-based compliant transmission mechanism}

When the sizes of flying robots fall into millimetre or even micrometre scales, the efficiency of conventional DC motors will substantially reduce. Furthermore, low friction pin joints are not feasible. Compliant mechanisms therefore become a natural solution for designing micro aerial vehicles. Considering the advancement in micro-electro-mechanical systems (MEMS) technology, developing complex and reliable micro-structures becomes possible. Having new technologies in materials will also allow to produce lightweight and controllable actuators. Many different kinds of alternative smart actuators have been suggested for flapping-wing MAVs.

A smart actuator is a micro mechanical device that generates motion using smart materials. Table 2.2 shows the main characteristics of the commonest smart actuators in terms of maximum free strain, maximum stress, deformation energy density, efficiency, and relative speed of response. As shown in the table, shape memory alloys (SMAs), shape memory polymers (SMPs), Electrochemo-mechanical Conducting Polymers (EMCPs), thermal actuators, mechano-chemical polymers (MCPs) can undergo large free strains and exhibit large blocking forces, but have slow response and limited efficiency, which make them not suitable for actuating a FWMAV. In contrast, piezoelectric actuators exhibit a relatively lower free strain. They are capable of producing very high blocking forces and more efficient sensitivity. Due to speed requirements, piezoelectric, dielectric elastomer, electrostatic, electromagnetic actuators are valid alternatives for FWMAVs.

Generally, smart actuators are integrated into compliant mechanisms and driven at their resonant frequency. Running at resonance can reduce inertial costs of acceleration and deceleration in the wings and will thereby improve efficiency in energy usage for FWMAVs ([6). Currently, the types of smart actuators that have been used together with the compliant transmissions towards resonance can be generally sorted into three categories, including piezoelectric actuator (15, 89, 90), electromagnetic actuator (22, 91), and dielectric elastomer actuators (25, 92). In the following subsections, a review of compliant transmission mechanisms for FWMAVs will be carried out according to the types of smart actuators used for driving.

\subsubsection{Piezoelectric actuators.}

Piezoelectric actuators are devices that make use of an inverse piezoelectric effect (90). The driving voltage for piezoelectric actuators normally ranges from tens to hundreds of volts. For 
Table 2.2: Overview characteristics of smart actuators. Source: (41).

\begin{tabular}{|c|c|c|c|c|c|}
\hline Actuator Type & $\begin{array}{c}\text { Maximum } \\
\text { Strain } \\
(\%)\end{array}$ & $\begin{array}{c}\text { Maximum } \\
\text { Pressure } \\
(\mathrm{MPa})\end{array}$ & $\begin{array}{c}\text { Specific } \\
\text { Elastic } \\
\text { Energy } \\
\text { Density }(\mathrm{J} / \mathrm{g})\end{array}$ & $\begin{array}{c}\text { Maximum } \\
\text { Efficiency } \\
(\%)\end{array}$ & $\begin{array}{c}\text { Relative } \\
\text { Speed } \\
\text { (full cycle) }\end{array}$ \\
\hline \multicolumn{6}{|l|}{ Dielectric Elastomer } \\
\hline (Acrylic) & 380 & 7.2 & 3.4 & $60-80$ & Medium \\
\hline (Silicone) & 63 & 3.0 & 0.75 & 90 & Fast \\
\hline Electrostatic & 50 & 0.03 & 0.0015 & $>90$ & Fast \\
\hline Electromagnetic & 50 & 0.10 & 0.003 & $>90$ & Fast \\
\hline \multicolumn{6}{|l|}{ Piezoelectric } \\
\hline$($ Ceramic $(\mathrm{PZT}))$ & 0.2 & 110 & 0.013 & 90 & Fast \\
\hline (Single Crystal(PZN-PT)) & 1.7 & 131 & 0.13 & 90 & Fast \\
\hline$($ Polymer $(\mathrm{PVDF}))$ & 0.1 & 4.8 & 0.0013 & 80 est. & Fast \\
\hline Shape Memory Alloy (TiNi) & $>5$ & $>200$ & $>15$ & $<10$ & Slow \\
\hline Shape Memory Polymer & 100 & 4 & 2 & $<10$ & Slow \\
\hline Thermal (Expansion) & 1 & 78 & 0.15 & $<10$ & Slow \\
\hline Electrochemo-mechanical Conducting Polymer & 10 & 450 & 23 & $<5 \%$ est. & Slow \\
\hline Mechano-chemical Polymer & $>40$ & 0.3 & 0.06 & 30 & Slow \\
\hline
\end{tabular}

example, popular monolithic stack actuators are available in voltage ratings from $60 \mathrm{~V}$ to 200 $\mathrm{V}$; for high-voltage discrete stacks actuators, their operating voltages ranging from $500 \mathrm{~V}$ to 1, $000 \mathrm{~V}$ are typical; the working voltage for the RoboBee mentioned below varies between 150 $\mathrm{V}$ and $300 \mathrm{~V}(\mathbf{1 8})$.

When coupled with mechanical transmission subsystems, the actuator can be capable of enhancing stroke amplitudes and reciprocating motions required for flapping flight (22). Piezoelectric actuators offer high displacements, fast response (17, 19) and high efficiency levels at high actuation frequencies (93). Therefore, piezoelectric actuators are frequently used for producing insect-scale flying robots.

Mateti et al. (15, 16, 94) developed an insect-scale air vehicle called LionFly, shown in Fig. 2.8 whose compliant flapping mechanism was manufactured monolithically from an epoxybased negative photoresist (SUEX) dry film. The prototype, with a mass $112 \mathrm{mg}$ was actuated by a PZT-5H bimorph actuator. Its resonant flapping frequency ranges approximately from 47.5 to $50.5 \mathrm{~Hz}$. The maximum amplitude for flapping can reach $75^{\circ}$. A lift to weight ratio of 1.5 was achieved by the prototype.

Sitti (89) has researched on piezoelectric actuators to drive a micro mechanical flying insect for stroke amplification. The piezoelectric materials, soft PZT and single crystal PZN-PT piezoelectric ceramics were used for fabricating the actuator. The piezoelectric actuator treated 


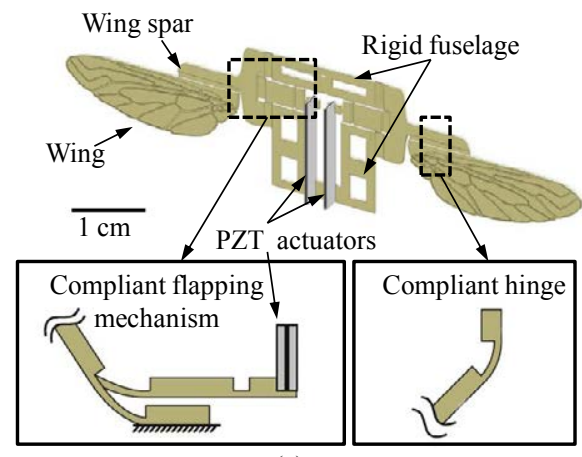

(a)

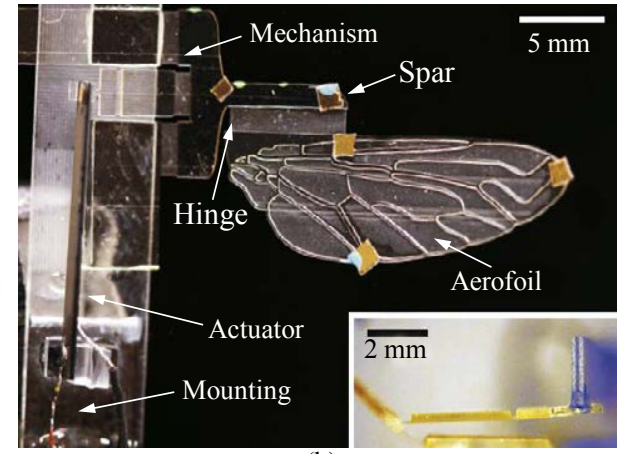

(b)

Figure 2.8: Robotic flying insect Lionfly at Pennsylvania State University. (a) Conceptual drawing of the LionFly. (b) A prototype LionFly with zoomed inset showing an end view of the flapping mechanism and actuator. Source: $(15,16)$.
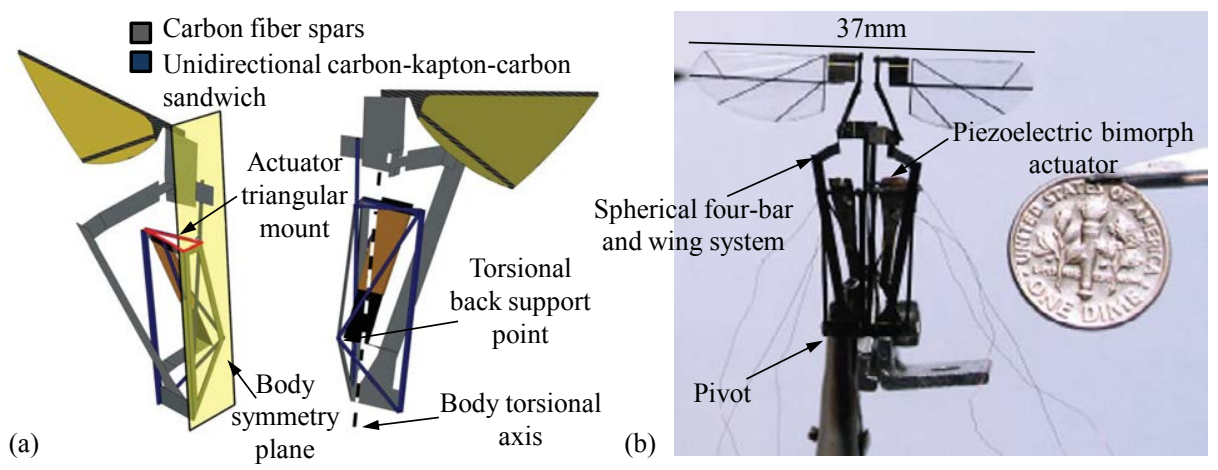

Figure 2.9: A spherical four-bar-wing system actuated by a bimorph piezoelectric bending actuator. (a) SolidWorks model of a four-bar-wing system. (b) Experimental setup for testing the performance of the spherical four-bar-wing system. Source: (17).

as a flexible part was compacted in a four-bar thorax mechanism as input link. The prototype of the compliant four-bar structure was made using laser micro machining and folding techniques. The stroke amplification of around 20-25 is achieved by the piezoelectric actuator-based four-bar mechanism. The wing can resonate at $29 \mathrm{~Hz}$ with around $90^{\circ}$ flapping motion.

Arabagi et al. (17) developed a spherical four-bar transmission mechanism actuated by a bimorph piezoelectric bending actuator driven in a quasi-static manner, as shown in Fig. 2.9. The piezoelectric actuator was made of two unidirectional M60J ultra-high-modulus carbon fibre layers sandwiched between two PZT-5H piezoelectric sheets. A passive extension out of two S-Glass layers was used for increasing the actuator stroke. A working prototype was manufactured via the smart composite microstructures technique to test and characterize the 


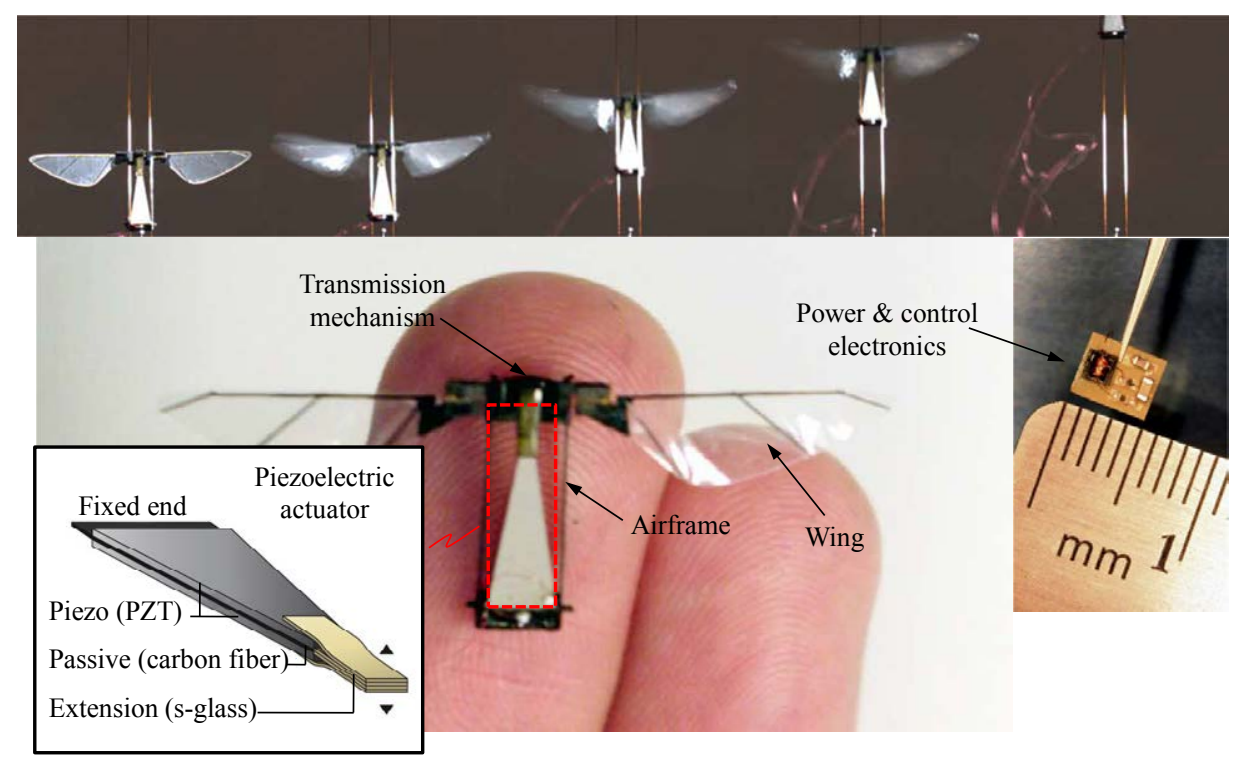

Figure 2.10: Harvard RoboBee driven by piezoelectric actuators. Source: $(18,19,20)$.

robot's lift production capabilities and the scaling law of the design with decreasing size. In (95), the authors presented a tool for designing flapping wing mechanisms using piezoelectric bending actuators.

Finally, Wood et al. at Harvard University (18, 19, 20, 96, 97, 98) have carried out intensive studies on an insect-scale flapping robot, called RoboBee, using a piezoelectric actuator. RoboBee was the first robotic insect capable of lift-off. The authors presented an innovative insect-scale robotic thorax design that is capable of producing an asymmetric wing kinematics that is similar to those observed in nature and used by two-winged insects in order to maneuver. Inspired by the thoracic mechanics of insects, a piezoelectric actuator has been fabricated as a bending bimorph cantilever actuator. The transmission maps the approximately linear motions of the actuators into flapping movements of wings. The transmission consists of links and joints with geometries designed to maximize the product of stroke amplitude and first resonant frequency, given that the actuator and airfoil properties are known. The insect-like robot, shown in Fig. 2.10, is capable of flapping at $110 \mathrm{~Hz}$. Actuators are created by laminating two piezoelectric plates (PZT-5H from Piezo Systems, Inc.) to a carbon fibre spacer and electrode layer as shown in Fig. 2.10. The airframe and compliant transmission are created by layering a $7.5 \mu \mathrm{m}$ polyimide film with carbon fibre face sheets. 
(a)

(b)
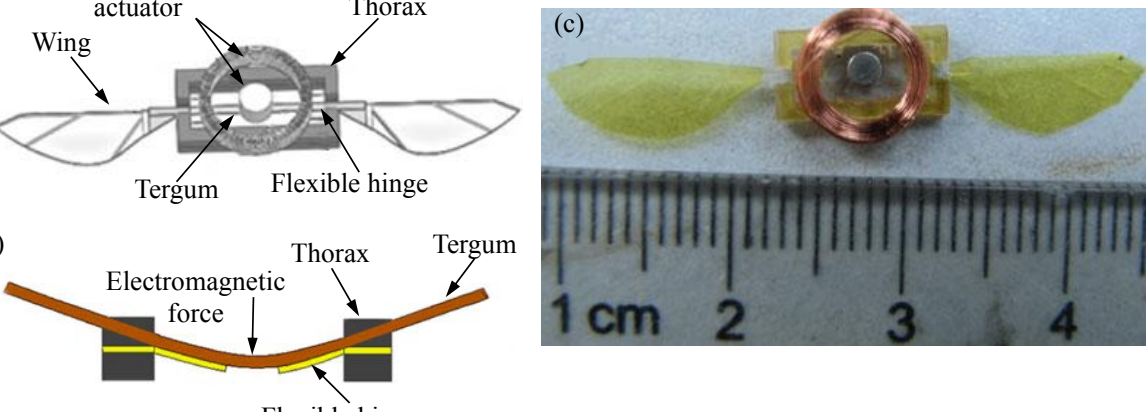

Flexible hinge

Figure 2.11: A 3.5-cm wingspan, $144 \mathrm{mg}$ weight prototype actuated by an electromagnetic actuator. (a) Top view of the structure. (b) The variation of wing' stroke with the effect of the electromagnetic force. (c) The physical prototype. Source: (21).

\subsubsection{Electromagnetic actuators.}

Electromagnetic actuators transform electrical and mechanical energy into each another by using the electromagnetic-mechanical principle. Electromagnetic actuators have a fast response, simple structure and are easy to control (21). Having very low voltage requirements is an advantage for using electromagnetic actuators. Their driving voltages can vary from 0 to $24 \mathrm{~V}$ (23).

Dargent et al. (91) presented a flapping structure concept at insect-scale based on MEMS technologies, which was actuated by a linear electromagnetic actuator. Based on this, Meng et al. (21) made a prototype FWMAV with 3.5-cm wingspan and $144 \mathrm{mg}$ weight (Fig. 2.11). Flexure hinges are used for occupying the gap between thorax and tergum (91) in order to avoid the energy loss and structure shocks. Two wings were also integrated in the prototype. According to the tests, the maximum flapping resonance frequency was $150 \mathrm{~Hz}$.

Finally, Deng et al. $(22,23,99)$ recently used a new electromagnetic actuator weighing 2.6 $\mathrm{g}$ to drive an insect-scale flapping-wing MAV at its resonant frequency, shown in Fig. 2.12. The electromagnetic actuator mainly consists of a single electromagnetic coil, a permanent magnet rotor, and a virtual spring magnet pair. Wing kinematics and mean lift measurements from the prototype demonstrated a lift-to-weight ratio grater than one at $24 \mathrm{~V}$. This framework was then extended to investigate the feasibility of the proposed actuator at different scales, predicting lift-to-weight ratios well above one for a wide range of the parameter space. Note that, strictly 


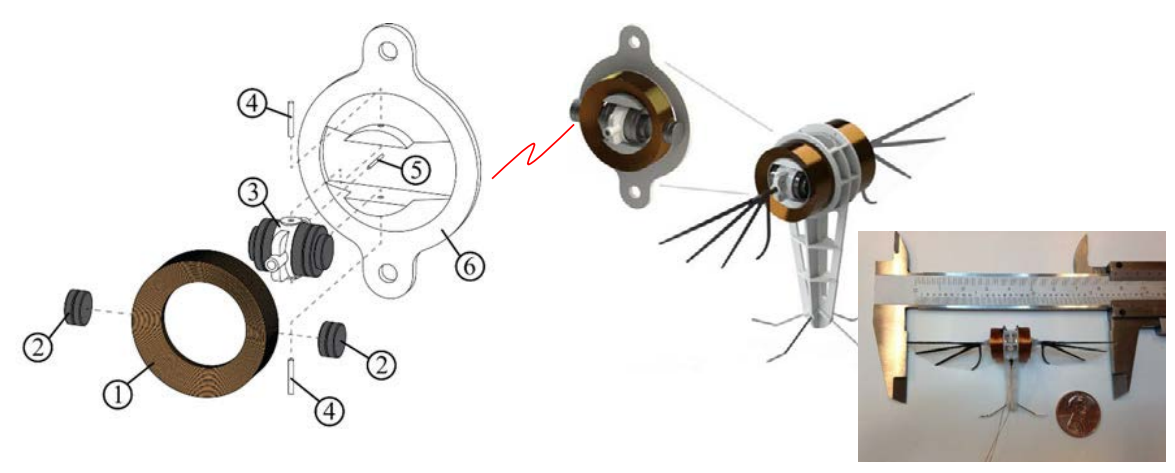

Figure 2.12: An electromagnetic actuator with flapping frame and experimental test. According to the left exploded view, the actuator consists of six components: (1) Wedge coil, (2) spring magnets, (3) rotor, (4) pivot pins, (5) stopper pin, and (6) frame. Source: (22, 23, 24).

speaking, this kind of electromagnetic actuator cannot be regarded as a compliant mechanism according to the definition of compliant mechanism given by L. L. Howell in (49), because they lack flexible components. The permanent magnets used in the prototype were attached to the perimeter of the coil to form a spring effect (23). These "virtual" springs created a restoring torque on the rotor when displaced from a neutral position, which is extremely similar to an equivalent joint (a revolute joint with a virtual spring) of a compliant joint in behaviour according to the PRB theory proposed in (49).

\subsubsection{Dielectric elastomer actuators.}

Dielectric elastomers (DEAs) are polymer materials with compliant electrodes that have a large electromechanical response to an applied electric field (41). DEAs normally operate at very high voltages $(\sim 1-10 \mathrm{kV})$, whose electric field is around $100 \mathrm{MV} / \mathrm{m}(100,101)$. They can produce large actuation strains at high work density.

In 2002, SRI researchers (102) conceived a thorax-type design using dielectric elastomers for a small flapping wing vehicle. Their research has shown that power densities of the silicone or acrylic elastomers used for driving flapping mechanisms could produce forces enough for lift-off. However, the structural support needed for actuators dramatically lowers the total power density because only 10-20\% dielectric elastomer film could be activated by the support structure.

In (92), the use for DEAs to drive an approximately 500g flapping-wing air vehicle is described. This prototype showed limitations in scaling up the muscles to the power levels re- 


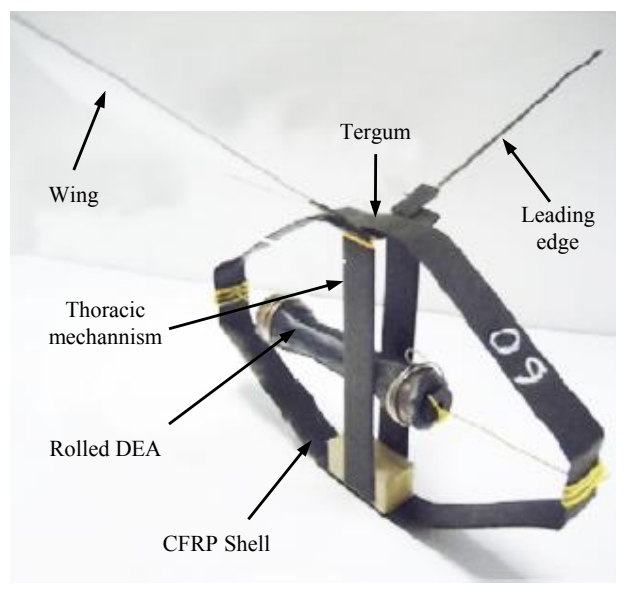

Figure 2.13: Integration of a CFRP shell and rolled DEA with a thoracic mechanism to form an insect-inspired wing flapper Source: (25).

quired for higher vehicle weight. Moreover, it was limited by the unavailability of batteries and microelectronics that could efficiently deliver the high voltage needed to drive the muscles.

In order to reduce the mass of the support structure and improve the overall work density of DEAs, Lau et al. (25) designed and developed a lightweight shell using a cross-ply laminate of carbon fibre reinforced polymer as a support shell structure to pre-strain a rolled DEA. A CFRP shell and a rolled DEA were integrated in an insect-inspired thoracic mechanism, which is shown Fig. 2.13. Research results showed that CFRP shell could achieve up to $35 \%$ axial prestrain to a rolled DEA. Furthermore, the structure could provide much higher of the maximum work density than the counterpart with spring rolls.

Table 2.3 shows the primary features of the prototypes reviewed in this section. As it can be observed form the table, most of current insect-scale FWMAVs are driven by piezoelectric actuators. Even though electromagnetic and dielectric elastomer actuators were adopted to actuate the FWMAVs, no evidences show that those developed prototypes can successfully lift off. Recently, an electrostatic actuator has been presented for artificial insect wings (103, 104). Such actuator consists of fixed electrodes and moving electrodes. The lift test of the artificial insect wing is presented in (103). The actuator works at a resonant frequency of 50-70 $\mathrm{Hz}$ excited by a DC power source with a voltage varying from $3 \sim 5 \mathrm{kV}$, without using any other complex AC circuits. 


\subsection{Flight Control Strategy}

Table 2.3: Comparison among various compliant flapping transmission mechanisms driven by smart actuators.

\begin{tabular}{|c|c|c|c|c|c|c|}
\hline Group & $\begin{array}{l}\text { Wing } \\
\text { Span } \\
(\mathrm{cm})\end{array}$ & $\begin{array}{c}\text { MAV } \\
\text { Weight } \\
(\mathrm{g})\end{array}$ & $\begin{array}{l}\text { Actuator } \\
\text { Type }\end{array}$ & $\begin{array}{l}\text { Compliant } \\
\text { Element }\end{array}$ & $\begin{array}{c}\text { Compliant } \\
\text { Stiffness }\end{array}$ & $\begin{array}{c}\text { Resonant } \\
\text { Frequency } \\
\quad(\mathrm{Hz})\end{array}$ \\
\hline Pennsylvania State Univ. & & & Piezoelectric & SUEX & & \\
\hline LionFly 89 & $7.2^{\mathrm{a}}$ & 0.112 & Actuator & Film & $26.8-107.4 \mu \mathrm{Nm} / \mathrm{rad}$ & $\sim 50$ \\
\hline Carnegie Mellon Univ. & & & PZT bimorph & PZT bimorph & & \\
\hline FWMAV (17) & 3.7 & 0.16 & Actuator & Actuator & $219 \mathrm{~N} / \mathrm{m}$ & 37 \\
\hline Harvard Univ. & & & PZT bimorph & PZT bimorph & & \\
\hline RoboBee(18, 19,20$)$ & 3 & 0.06 & Actuator & Actuator & - & 110 \\
\hline Shanghai Jiao Tong Univ. & & & Electromagnetic & SU-8 & & \\
\hline FWMAV 21 & 3.5 & 0.143 & Actuator & Film & - & $120-150$ \\
\hline Purdue Univ. & & & Electromagnetic & Virtual & & \\
\hline FWMAV (22 23 99) & $9-14$ & $5.2^{\mathrm{b}}$ & Actuator & Spring & $0-5.64 \mathrm{mNm} / \mathrm{rad}$ & $30-71$ \\
\hline $\begin{array}{c}\text { Nanyang Technological Univ. } \\
\text { FWMAV }(\underline{25})\end{array}$ & $13^{\mathrm{c}}$ & $\begin{array}{c}10.5 \text { (without } \\
\text { electrical devices) }\end{array}$ & $\begin{array}{c}\text { Dielectric Elastomer } \\
\text { Actuator }\end{array}$ & $\begin{array}{l}\text { CFRP } \\
\text { Shell }\end{array}$ & $102^{\mathrm{d}} \mathrm{N} / \mathrm{m}$ & $5-10$ \\
\hline
\end{tabular}

a Estimated from an image of the LionFly's conceptual drawing in Ref. (16).

b Estimated based on two actuators and each one is with a wing.

c Calculated using $2 \times$ wing length $+1 \times$ CFRP shell width $=2 \times 6 \mathrm{~cm}+1 \times 1 \mathrm{~cm}$.

d CFRP shell contributes one quarter of the total axial stiffness, $408 \mathrm{~N} / \mathrm{m}(25)$.

\subsection{Flight Control Strategy}

Control strategies for FWMAVs can be classified into two categories: linear control and nonlinear control. These two aspects are reviewed in this section. In addition, advanced control techniques, like adaptive control and fuzzy neural network control, which are believed to be possibly the best candidates for flight control over a wide range of operating conditions, are also briefly introduced here.

\subsubsection{Linear Control}

Oppenheimer et al. (105) presented the modelling and control of a flapping wing micro-air vehicle based on the Harvard RoboFly presented in (19). The aerodynamic model used in their research was developed in (106). In the vehicle, three actuators are used, among which two are used to independently vary the position of the wing spars in the stroke plane and the rest is used to control the position of a bob-weight. The authors presented a 5-DoF control of the fuselage. There are two kinds of input parameters for this controller. One includes the the fundamental wing beat frequencies, which can change the frequency of the temporally symmetric portions 
of the wing beat. The other consists of the split-cycle parameters, which are used to vary the temporally asymmetric portions of the wing beat. Later, the work described in (105) is further expanded. In (107), only two physical actuators are used. The new split-cycle constant-period frequency modulation can provide independent control over vertical and horizontal body forces as well as rolling and yawing moments. A cycle-averaged control law has been successfully verified in two cases of aerodynamic models. One is based on blade-element theory while the other is an unsteady aerodynamic model derived from experiments. After that, wing bias $(108,109)$ are added in the model in (107). Wing bias shifts the mid-point of the wing stroke, enabling six degree of freedom control and the generation of asymmetric lateral forces. The addition of wing bias also increases the number of control inputs to four from the previous model using only split cycle frequency modulation.

Deng et al. (110) used the standard aircraft model and time-averaged forces and moments for a biomimetic insect. A switching controller was derived and successfully applied for the insect model under a hove condition. After that, Deng et al. (56) developed flight control algorithms for flapping wing micromechanical flying insects (MFIs). In their research, the timevarying dynamics of insect flight caused by the flapping wings was approximated with a timeinvariant system based on averaging theory and wing trajectory parametrization. The dynamic model was treated as a discrete-time dynamical system and then a closed-loop identification was implemented to estimate the system. Finally, Using averaging and linear quadratic regulator theory, a control strategy for ensuring proper stroke kinematics is presented resulting in good tracking of a reference flight condition.

Cheng et al. (111) derived linear dynamics and control of a model insect near hover. The body of the model insect is modelled as three rigid ellipsoids based on the morphological data of fruit fly Drosophila. Each wing of the insect has two DoFs, i.e., stroke and rotation motions. For simplification, the stroke deviation is neglected. To analyse the near-hover flight dynamics of the model, six-DoF Newton Euler equations with translational and angular velocities were linearised using small perturbation theory. For eliminating the effect of translational component of velocity, the flight dynamics are confined to the 3-DOF angular motions. The stability derivatives are estimated by using flapping-counter forces and flapping-counter torques based on quasi-steady aerodynamic model in (112). Their research shows that the attitude dynamic system could be regulated stably using a proportional feedback controller with proper parameters while the open loop system can not because passive damping cannot tune the insect model back to the original orientation. 
Xiong et al. (113) conducted the stabilization control to both hovering an forward flight of bumblebee flight previously developed in (114, 115). A computational fluid dynamics (CFD) method was employed to compute the flows and obtain the control derivatives. Then, the techniques of eigenvalue and eigenvector analysis and modal decomposition were used to study the stabilization control of the insect. In their research, for all of the forward flight speeds presented in their research, the system is controllable. In addition, they also attempted to explain the reasons why the bumblebees can fly stably even if they are passively unstable.

Geder et al. (116) presented a flapping-wing model with a $7.5 \mathrm{~cm}$ wingspan, $10 \mathrm{~g}$ mass and no tail. Shape alloy memory actuators are employed to change wing parameters in terms of wing stroke amplitude, wing stroke mean position, and wing stroke plane angle. Stroke amplitude for each wing was used to control vertical and lateral motion, and functional roll. Stroke mean position was used to control forward motion and pitch. Stroke plane angle was used to control functional yaw. Sensors were equipped on the model for feedback control of the vehicle dynamics. Control for hovering, forward flight, and turning maneuvers was achieved through the use of PID-control and an extended Kalman filter.

\subsubsection{Nonlinear Control}

Besides linear control, investigations on nonlinear control have also implemented on the stabilization of MAVs. Serrani et al. (117, 118) expanded a six-DoF control mentioned in (107, 119) into a robust control. In(117), Serrani presented a robust hovering control of a FWMAV with one DoF. The purpose of the controller is to ensure that the trajectory of a periodic orbit could be asymptotically stable about the desired point. After that, Serrani et al. further extended his work to control a 3-DOF FWMAV. In (118), a 6-DoF dynamic model proposed by Bolender in (120) was employed and motions of the model were restricted to the longitudinal $\mathrm{x}-\mathrm{z}$ plane. To illustrate the specific issues accompanying with the control, two actuation forms were taken into consideration, i.e, variable wingbeat frequency and stroke plane angle. Meanwhile, the state of vertical dynamics were augmented with the set-point tracking error, as in (117). Through the use of decomposing the system dynamics into different time scales, the $\mathrm{x}$ and $\mathrm{z}$ positions of the FWMAV can be controlled directly and the pitch attitude can also be stabilized. Simulation results show the control methodology is effective because the model can be achieved the set tracking point in about one minute.

Rifai et al. (121) introduced a bounded nonlinear state feedback control of a flapping wing MAV in three dimensions. The dynamic model is based on the standard aircraft equations of 
motion. Authors adopted quaternions to replace the widely used Euler angles for the description of the orientation, in order to reduce the computation complexity and avoid numerical singularities caused by Euler angles. The aerodynamic forces and torques used in the model are obtained according to the averaging theory. The proposed controller could stabilize the attitude of the FWMAV under the presence of external disturbances. Humbert and Faruque expand the analysis presented in (122) to a reachability analysis in (123). After simulation, the controllability is achieved through carefully selecting control inputs: flapping magnitude change, stroke plane change, flapping offset, and change in the angle of attack between the upstroke and downstroke.

Researchers at Harvard Microrobotics Laboratory recently applied adaptive control (62) to their nano-scale RoboBee to achieve fundamental autonomous flight. In (62), Chirarattananon et al. proposed an adaptive flight controller to cope with uncertainties in the system. Before taking flight experiments, authors assumed that the robot orientation is always upright and the produced thrust force is always along with the vertical axis. Flight control experiments were performed in a flight arena with a volume of $0.3 \times 0.3 \times 0.3 \mathrm{~m}$, in which eight motion capture vicon cameras were fixed to provide position and orientation feedback for the controller. During the hovering flight tests, the controller can not timely respond the commands and there exists a $0.2 \mathrm{~s}$ delay after the controller is activated. However, the time delay seemingly has no effect on the control and the robot can finally converge to the set position. Experimental results demonstrates that the adaptive controller overpasses a similar non-adaptive approach in case of sustained hovering flights with relatively small errors. In addition, vertical takeoff and landing flight experiments were also carried out to verify the reliability of the proposed controller. Furthermore, Duan et al. (124) derived the dynamic model of FWMAVs in detail and put forward two methodologies to control the attitude of a FWMAV. One is an adaptive robust controller (124) while the other is a sliding mode adaptive controller (125). Both of their stabilities were analysed and presented in the two papers.

Considering the unsteady aerodynamics in complex flight environments, a neural-network or learning-type control concept would have more advantages in adaptability and robustness than traditional control methodologies as stated in $(64,65)$. In this regard, fuzzy neural networks have been investigated for control schemes for flapping wing micro-air vehicles. Guo et al. (126) developed a fuzzy neural network controller to achieve high maneuverability of a biomimetic FWMAV through adjusting mid-stroke angle of attack and flip start timing. The longitudinal dynamics of the biomimetric robot is based on the work presented in (127). According to their 


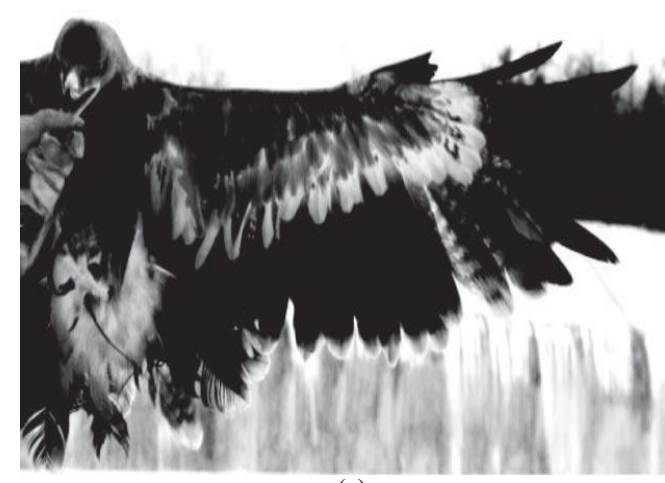

(a)

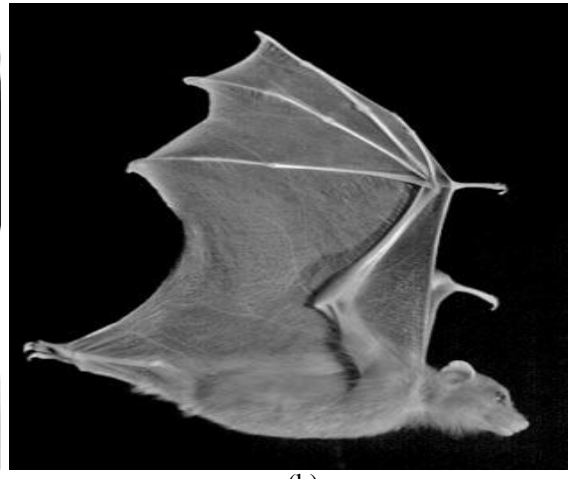

(b)

Figure 2.14: (a) Schematic of the relative arrangement of the ten wing bones of a bird. The geometry of the bones is intended to be indicative (26, 27). (b) The wing of a lesser dog-faced fruit bat $C$ ynopterus Brachyotis comprises an elastic muscularized membrane that is stretched between the elongated digits of the hand, the hindlimbs, and the body wall. Source: (27).

simulation, the fuzzy neural network controller could get the position and orientation stabilized along the desired trajectory. Other control techniques include control through engineered central pattern generators in (128) and the evolution of analog neuromorphic devices in (129).

\subsection{Morphing-wing Concepts for Flapping wings}

\subsubsection{Bio-inspiration}

In nature, flying animals (e.g. birds and bats) own the unparalleled capability of maneuvering in complex environments, which is far better than the counterpart of man-made aerial vehicles. One dominant advantage of these flying animals is that they can timely morph the configurations of their wings during flight to adapt to external environments (130, 131).

Bird wings are anatomically homologous to human arms in which they have portions corresponding to an upper arm, lower arm, and hand, as shown in Fig. 2.14(a). The wing connects to the body through the shoulder joint, which is activated by several muscles. The elbow and hand joints are located distally and form a V-shape under muscular control. During flight, birds can manipulate these joints to adjust wing sweep and independently fold each wing. Birds can tuck their wings against the body through the musculoskeletal control of their overlapping feathers while maintaining a high performance aerodynamic shape (132, 133). Such extreme wing morphing allows for impressive feats of maneuverability, such as flying through brush with gaps barely larger than the body (33). 


\subsection{Morphing-wing Concepts for Flapping wings}

The structures of bat wings are much more complex than those of birds, as shown in Fig. 2.14(b). Bat wings have more than two dozen of independently controlled joints and more than thirty degrees of freedom of motion can be realized. Bat shoulder possesses a mobile scapula with more 20 different muscles that are responsible for the overall motions of the wing, e.g., the wingbeat amplitude and stroke plane (38). The wing folding and expanding are controlled through the contraction and extension at elbow, and abduction and adduction at wrist. Combining those movements of shoulder, characteristic traces of wing tips will be emerged in 3D space (134, 135, 136). The remaining joints within the digits could change the dynamic shape of the wing in camber. These articulated skeletons of wings are covered by the flexible membranes under active muscular control (137, 138) to morph wings. The distinct arrangement of the bat wing suggests a high potential capability to adjust the wing morphing according to aerodynamic demands in flight (27).

To mimic the abilities of wing morphing musculoskeletal control in birds and bats, a number of bio-inspired robots have been developed. Among these robots, the bio-inspired morphingwing structures can be categorized into three types (33): (1) non-flapping wings that morph, (2) passive morphing wings that flap with a compliant structure which passively morphs through aeroelastic coupling, (3) active morphing wings that flap with an active actuating system to morph the wing shapes in conjunction with a part of passive aeroelastic deformation. The first non-flapping but morphing wings usually appear in miniature air vehicles propelling by using a propeller. For example, a multiple-joint morphing-wing robot from the University of Florida could sweep both arm and hand wings through biomimetic shoulder and elbow joints inspired by a seagull's wing (139). The changes of wing shape and area can be achieved by controlling the angular positions at shoulder and elbow joints (140). This morphing actions can reduce turn radius and better reject crosswinds (33). Because these non-flapping but morphing wing robots have been reviewed in Ref. $(54,66,70)$ and further they don't belong to the category of FWMAVs, we will not discuss these kinds of flying robots in this paper. In the following, more attention will be paid to bio-inspired morphing concepts applied on FWMAVs. Hopefully, this review can give readers an overall sight on the current status of morphing-wing FWMAVs.

\subsubsection{Passive Morphing Wings}

For traditional flapping-wing MAVs, the geometrical areas of wings' shapes are constant and invariant during both up and down strokes while flight. Flapping motions during upstroke will generate negative lift forces, which are harmful for flight. The adverse forces produced during 

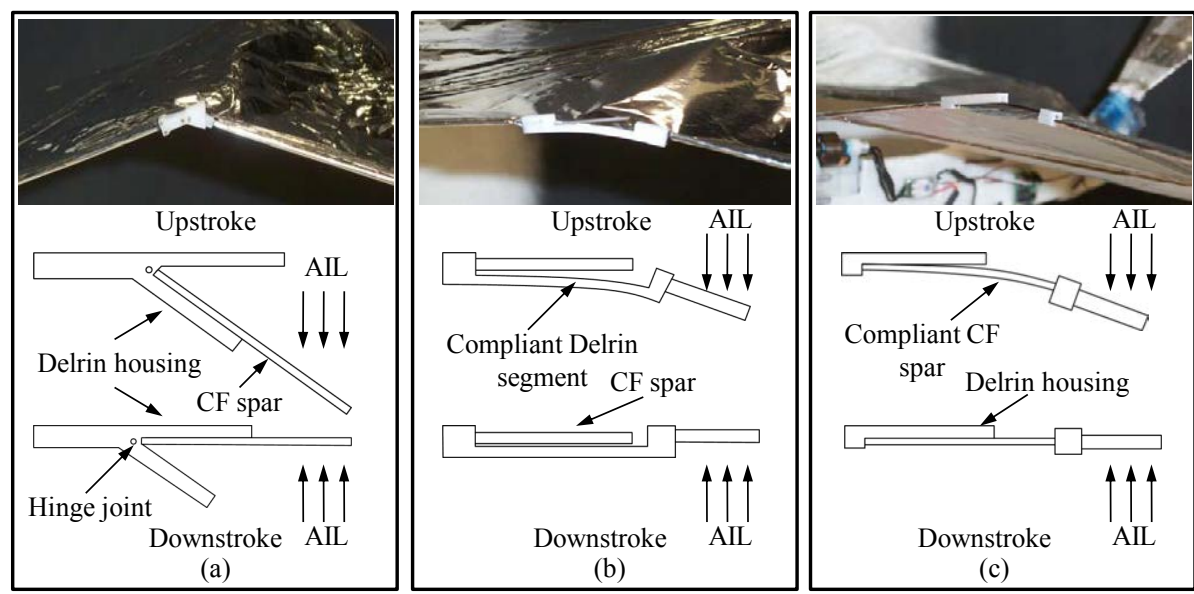

Figure 2.15: Three types of passive morphing wing concepts and their responding morphing principles with effects of aerodynamic and inertial loads (AIL). (a) Rigid passive morphing concept with a angular limitation of wings' motion. (b) Flexible Derlin hinge Concept. (c) Compliant carbon fibre (CF) hinge concept. Source: (28).

upstroke need to be reduced and minimized for improving flight efficiency. In nature, biological flyers can always give us some inspirations. Large birds exhibit excellent flight behaviours on tailoring the wing flapping. They fold their wings in toward their bodies during the upstroke for lowering the air resistance. The consequent is that the magnitude of harmful negative lift forces are reduced as the wing area is decreased. In contract, during downstroke, birds fully re-extend their wings and increase wings' areas to produce more useful upward forces comparing to the upstroke. Obviously, if the style of flapping can be integrated into the design of the FWMAVs, the overall lift forces will be enhanced over a whole flapping cycle and to some extent to lower the dependence on aerodynamic lift generated by forward flight. Therefore, the concept of passive morphing wing is proposed based on this point.

In Ref. (28), Mueller et al. introduced a successfully flying miniature air vehicle whose wings were fabricated with one-way compliant passive morphing mechanism for realizing the folding style like large birds. The authors put forward three types of one-way compliant mechanisms mounted on the leading edge, which are shown in Fig. 2.15. Fig. 2.15(a) is a tree-branchlike rigid passive morphing concept, which restricts wings' motions in a desired angular range. During upstroke, with the effects of aerodynamic and inertial loads, the outboard portion of the wing carbon fibre (CF) spar swivels downwards around the hinge joint mounted in the Delrin housing, finally arrives to the maximum folding angle and maintains the angular position to the 


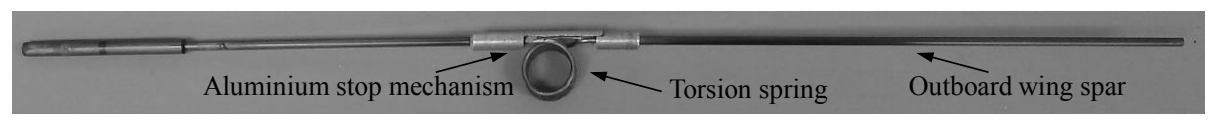

Figure 2.16: Starboard passively morphing wing spar. Source: (29).

end of upstroke. In the subsequent downstroke, aerodynamic and inertial loads push it upwards and the wing starts to unfold. When the outer part hits the delrin housing, a hard stop of the wing will happens. Wing unfolding is also terminated and keep the wing fully extended in the rest stroke. This mechanism allows to vary the range of the wing folding according to design requirements, but the undesirable hard shock during every flapping cycle can't be eliminated. As shown in Fig. 2.15(b), the second concept used a flexible Delrin segment as the compliant element. The compliant Delrin segment affords the desirable bending to fold for reducing aerodynamic forces produced by wings during upstroke. During downstroke, the central carbon fiber spar prevents the undesirable deformation of the leading edge to fold for generating more lift forces. This distributed bending along the length of the Delrin flexible member can farthest minimize the sharp shock of the former design. The third design in Fig. 2.15(c) is almost the same idea as the second one. The main differences are that the installation location of the Delrin housing is vertically reversed and the compliant Delrin part is also replaced by a carbon fiber spar with a smaller diameter. The purpose of this arrangement is to increase the bent angle of a wing during upstroke. Their research results demonstrate that, the aerial vehicles with morphing-wing designs don't compromise the overall lift and can maintain the payload carrying capacity at the expense of reducing forward flight velocity relative to those with non-morphing wings.

Billingsley et al. (29) did a similar style of wings on a modified version of the Park Hawk series of ornithopter, shown in Fig. 2.16. Passive torsional springs were installed in the primary wing spar at approximately half the length of the wing. An aluminium stop mechanism bridges the gap over the torsional spring which allows the outboard wing spar to bend down during the upstroke, but not up during the downstroke. The carefully designed wings were used to exploit the flight performance when the wings' areas were reduced during upstroke. Compared to the ornithopter with non-folding mechanism, the vehicle employed the passive morphing-wing mechanism had a much higher net lift while the thrust force produced was greatly reduced, which dramatically threaten the capability of forward flight. 


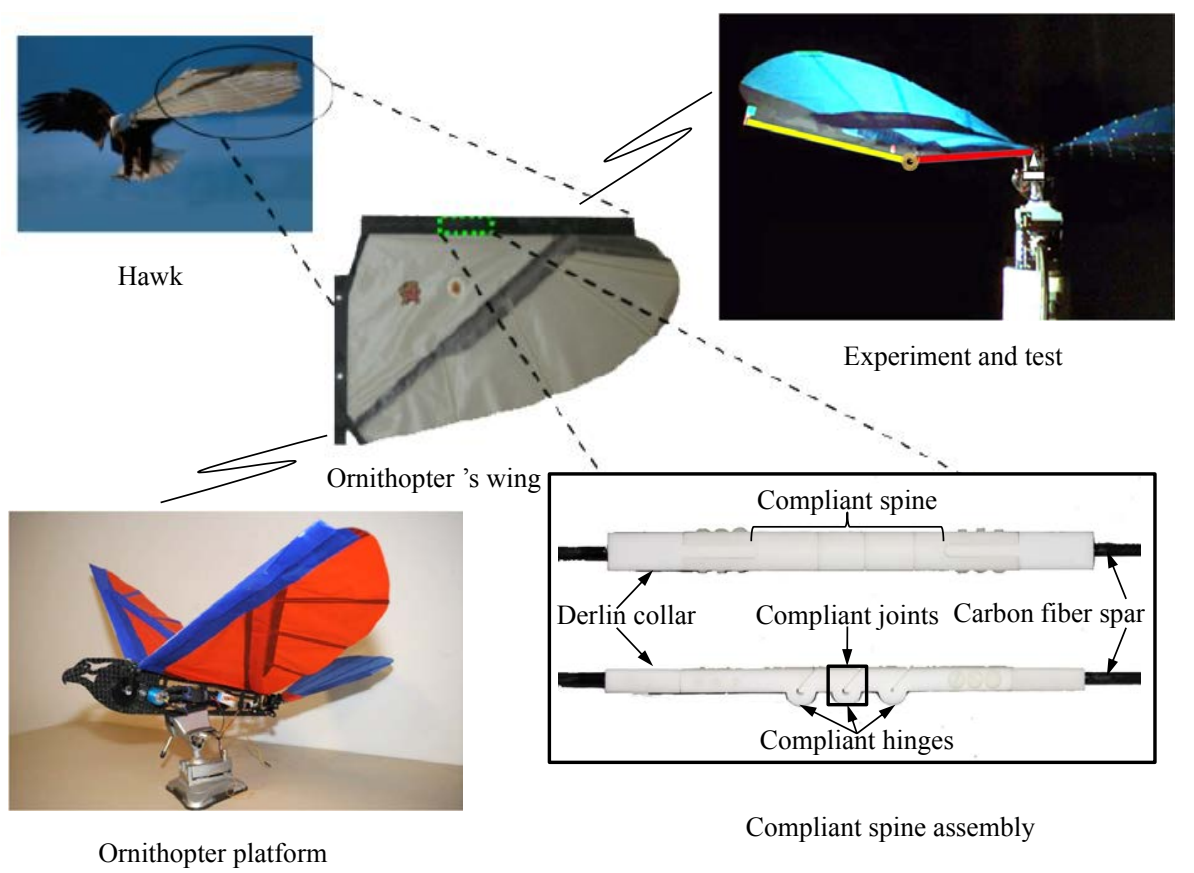

Figure 2.17: A test ornithopter with passive morphing wings. A compliant spine, which is designed to be stiff during downstroke and flexible during upstoke, is inserted in the leading edge spar of the ornithopter for investigating its influence on the flight performance of the vehicle. Source: $(30,31,32)$.

To relieve the thrust expense and enhance the whole flight performance of the ornithopter, more sophisticated design and analysis on wings are needed. The Morpheus Laboratory researchers at University of Maryland have done some improvements on the basis of the Billingsely et al.'s research. In Ref. (30), Tummala et al. did the design and optimization of a novel compliant spine for passive morphing wings of the ornithopter, in stead of the torsional spring used in Ref. (29). In Ref. (31), Wissa et al. continued their former work on design and optimization in (30). Besides that, the flight tests were also conducted to assess the steady level flight performance of the test ornithopter with various integrations of compliant spines by using vicon motion tracking cameras. A general view of compliant spine assembly and the test prototype is displayed in Fig. 2.17. Test results demonstrate that incorporating compliant spines into the leading edge spars of wings can lower the positive acceleration at the body's center of mass and the reduced values are translated into overall lift gains. In Ref. (32), Wissa et al. focused on the structural stability analysis on the leading edge spar in which a compliant spine is inserted, 

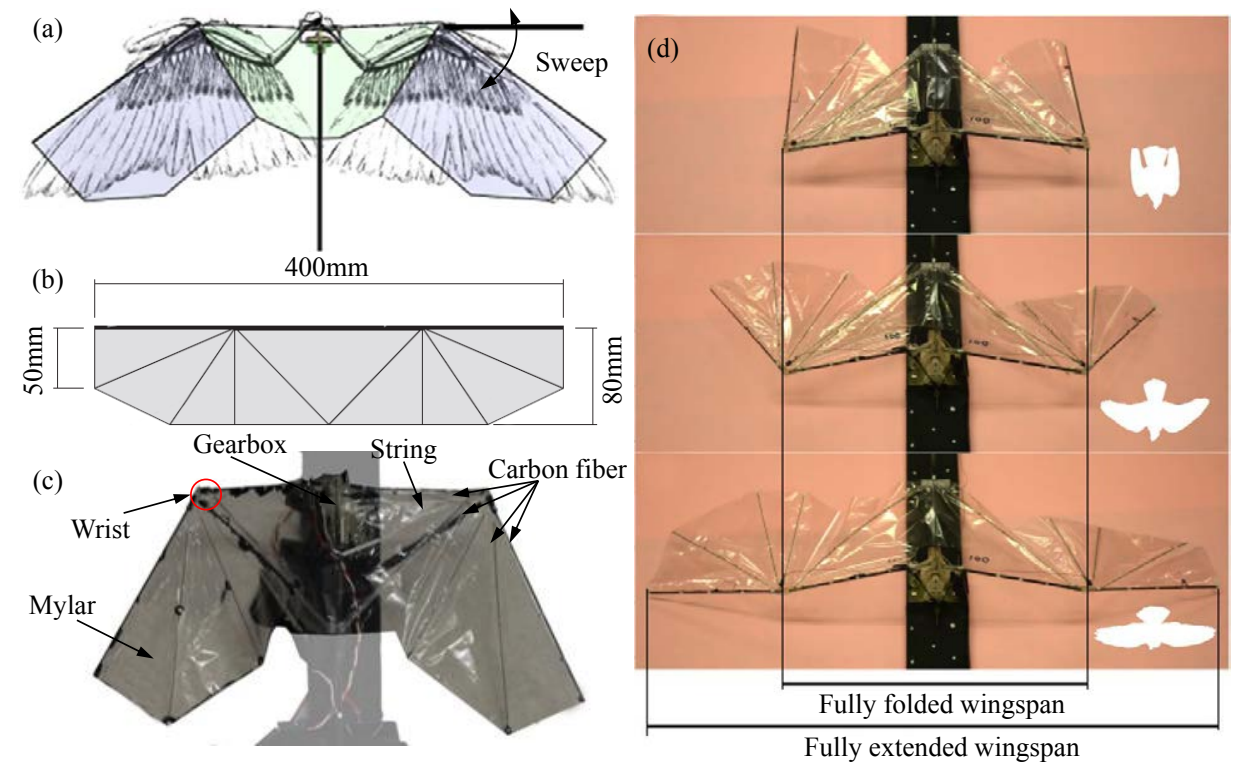

Figure 2.18: A passive wing morphing inspired by bird wing morphology. (a) Comparison between the robotic wing and bird wing morphology. Both bird and robotic wings could rotate their wrists(hinge) to sweep forwards (unfold) and backwards (fold). (b) The mechanical sizes of the robotic wing. (c) Wings were constructed with carbon fibre leading edges and spars, Mylar foils and other 3D printed joints. Strings constrain the wing from unfolding until released. (d) Variation of the robotic wing in static configuration. (Top) fully folded, (Bottom) extended and (Middle) partially extended. Source: (33).

and a model of the leading edge spar-spine with a torsional viscous damper. Experimental data supports the validation of the theoretical model. In addition, experiment also express that a compliant spine design placing at about $37 \%$ of the wing half span could make the leading edge spar-spine structure keep stable and there exist a safety range of damping ratio in which the leading edge spar-spine system is stable for all the stiffness values of compliant spines.

Stowers et al. (33) presented a passive wing morphing mechanism mainly inspired by the overlapping feathers of birds, shown in Fig. 2.18. The mechanism consists of an unactuated hand wing connected to the arm wing with a wrist joint. Their purpose is to investigate the unfolding behaviour of wings from a folded position and the special situations when wings deflected by a colliding obstacle, only with the action of wings' periodic flapping motions and without additional actuation. Research results indicate that unfolding behaviours of wings are dominated by centrifugal accelerations generated by flapping motions rather than aerodynamic or gravitational forces, which is different from the conclusion that passive morphing is caused by aerodynamic forces in Ref.(28). 


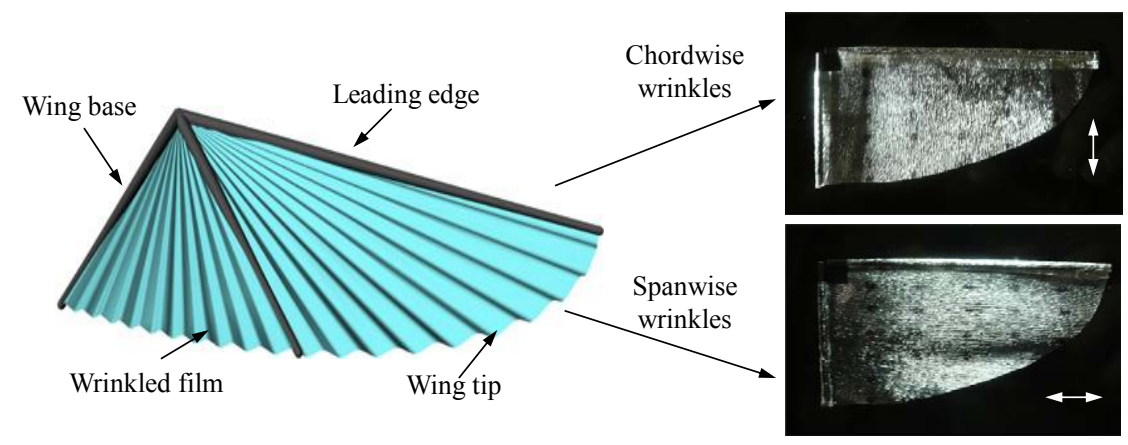

Figure 2.19: Bio-inspired wrinkled wings for micro flapping-wing aerial robots. Source: (34).

In addition, a novel self-organized microwrinkle membrane concept for passive morphing wings was proposed by Tanaka et al (34) inspired by bird feathers and the corrugations of insect wings. The wing is made of carbon fibre-reinforced plastic (CFRP) frames and a polymer film with microscale wrinkles, including spanwise wrinkles and chordwise wrinkles two types, as shown in Fig. 2.19. The wrinkle film wing is mounted on the rigid wing frame and it can passively morph with an effect of aerodynamic force. Experimental results show that, the chordwise wrinkle wing can result in much greater twist along the leading edge, and meanwhile produce more lift than the spanwise wrinkle wing. Even through some defect exists, e.g., the absent experimental support on the reduction of tensile stiffness using microwrinkles, however, the design method provides us a new thought on the fabrication of morphing-wing structures.

\subsubsection{Active Morphing Wings}

Currently, the study of active morphing-wing concepts mainly focuses on the fixed- and rotary -wing aircraft. There exist very few active morphing-wing concepts that are incorporated into a flapping-wing aerial platform due to the strict weight constraints present at micro sizes. Using conventional DC motors to actively morph the wing shape seems to be not feasible because DC motors and required on-board control units that synchronize both flapping and morphing motions will add additional weights. The development of smart materials opens a door for the research of active morphing-wing structure on FWMAVs. Using smart materials can reduce complexity and improve the reliability of the system. The weight saving and the possibility of using smart materials as both actuators and sensors within the structure are potential advantages (75). Accompanying with the inspiration of natural animals, several pioneers have already started their steps to design and fabricate the morphing-wing FWMAVs. 


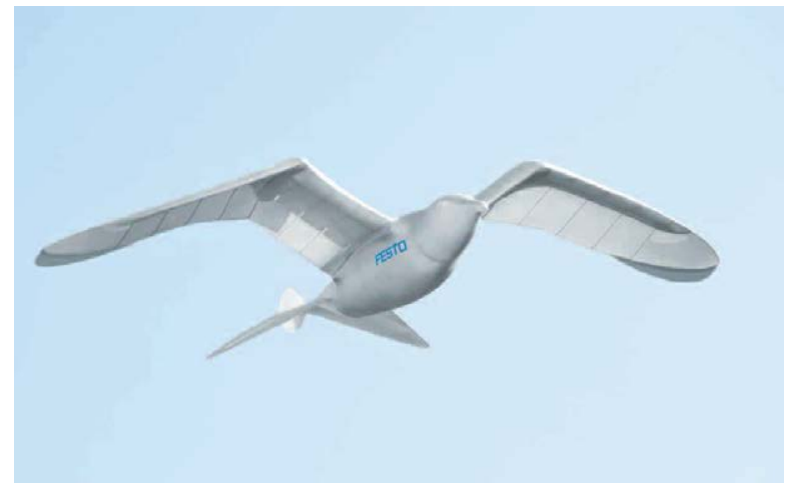

Figure 2.20: Festo SmartBird.

Festo SmartBird (141, 142) is a gull-inspired flapping-wing UAV with active morphing wings that has successfully flown. As shown in Fig. 2.20 , it has a $2 \mathrm{~m}$ wingspan and a more than $1 \mathrm{~m}$ body. Each wing consists of a two-part arm wing spar with an axle bearing located on the torso, a trapezoidal joint as is used in enlarged form on industrial excavators, and a hand wing spar. The arm wing generates lift while the hand wing beyond the trapezoidal joint provides propulsion. Both the spars of the inner and the outer wings are torsionally resistant. The active torsion is achieved by a servomotor at the end of the outer wing which twists the wing against the spar via the outmost rib of the wing. When SmartBird lifts its wings, the servo motor for active torsion twists the tips of the hand wings to a positive angle of attack, which is then changed to a negative angle a fraction of a wing beat period. The angle of torsion remains constant between these phases. Through this active articulated torsional drive in conjunction with a complex control system, an energy-efficient flight can be realized for the adaptation of the natural model. However, scaling the large body down is a big challenging in order to accomplish missions in compact spaces.

BATMAV (35, 36) is a robot to reproduce bat-like flapping flight capabilities. The prototype is shown Fig. 2.21. A kind of smart material named shape memory alloy (SMA) was chosen as muscles on wings. Here, the SMA material plays a dual role. One is that the SMA wires were treated as actuators to drive wings to realize flapping and morphing motions like bats while the other is that SMA wires at joints were regarded as flexible hinges to fully use the capability of super-elasticity. The kinematic analysis and simulation of wings' trajectories were carried out by using Matlab. Even though simulation results showed that the wing could realize similar trajectories as real bats, however, the lack of practical experimental support may threaten the reliability of the model. Meanwhile, the most important aerodynamic tests were also missing. 


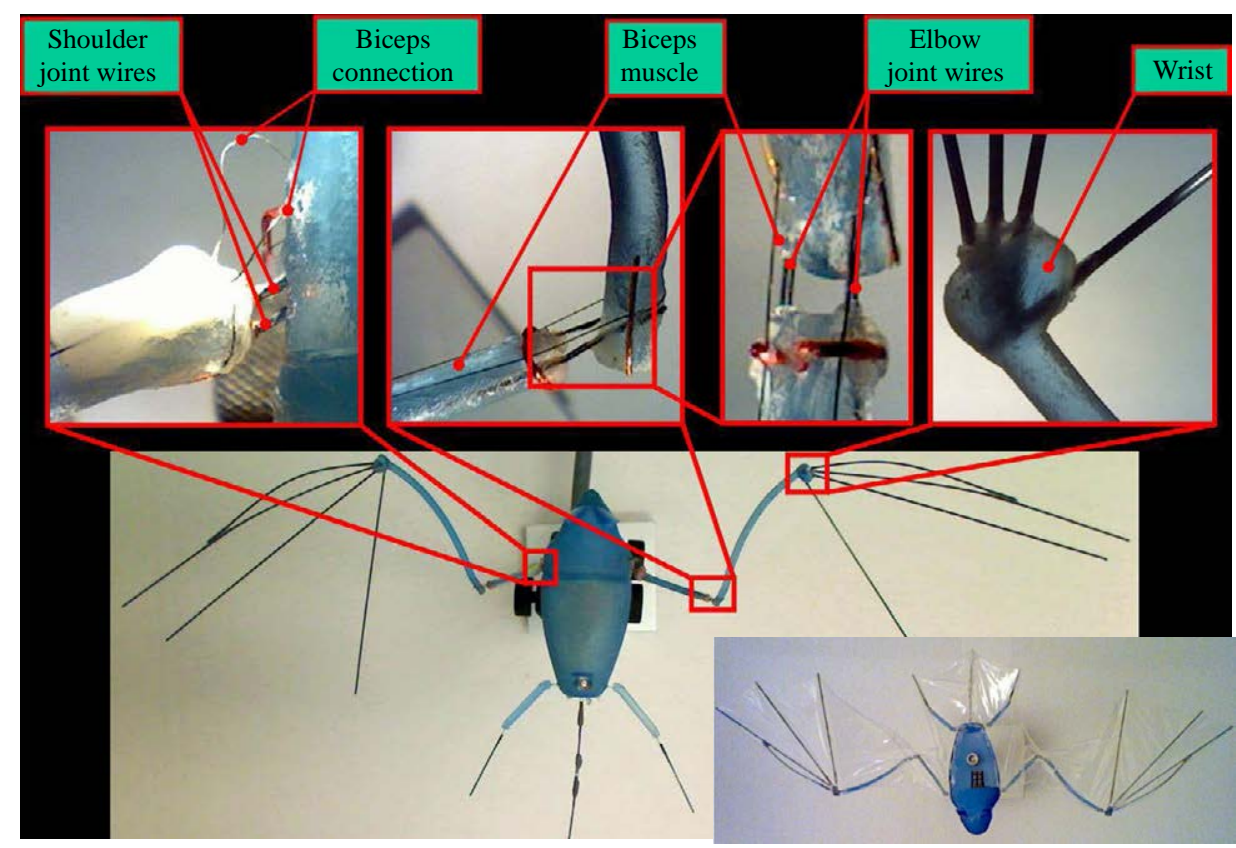

Figure 2.21: The BATMAV actuated by using SMAs wires for flapping and morphing like bats. Source: (35), (36, 37).

The research presented in (38) is aimed to design and fabricate a batwing flapper to obtain data that is difficult to get from a real flying bat. The flapper has captured the main skeletal structure based on the relative dimensions from the anatomy of bats named Cynopterus Brachyotis. The prototype of the robotic batwing flapper is shown in Fig. 2.22. In Ref.(39), the same authors extended their research on the relationship between the energetic cost and those different kinematic parameters in terms of wingbeat frequency and amplitude, stroke plane angle, downstroke ratio, and wing folding.

The study on the batwing flappers also appears in E. Garcia's work. In Ref. (143), authors presented design and fabrication of bat-wing flappers on the basis of the biomechanics of bat's wing. Several different materials of membrane were assembled. Also, the wing performance in terms of the lift-to-drag production was analysed and test in the wind tunnel. Besides that, a second research from the same authors in Ref. (144) concerned on key characteristics of bat flight for fully understanding how certain aspects of bats' wings could affect the fight and meanwhile considering whether it was possible to integrate the inspirations from bats into the design of future MAVs. Here, the aspects of bats' wings include morphing, cambering and twisting. The lift and drag productions on each aspect behaviour were measured and quantified. 


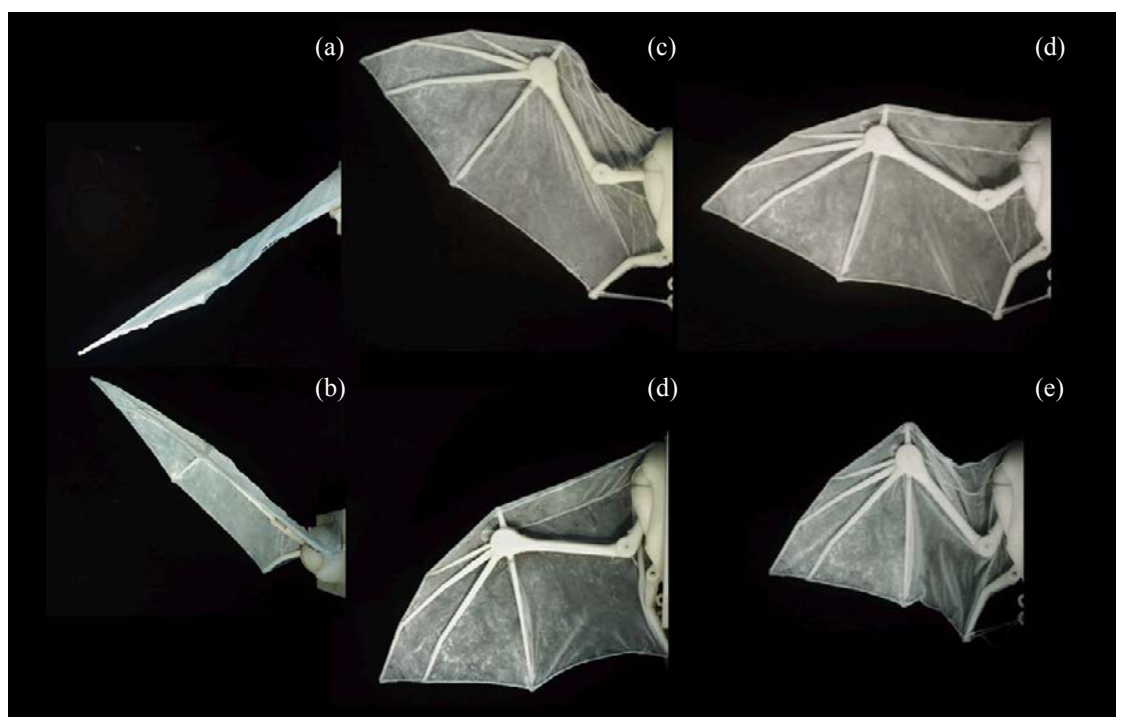

Figure 2.22: Different wing configuration of a robotic batwing flapper with kinematic values closest to median values of model species: $8 \mathrm{~Hz}$ wingbeat frequency, $75^{\circ}$ wingbeat amplitude, $60^{\circ}$ degrees stroke plane angle, 0.45 downstroke ratio, and $40^{\circ}$ wing folding. Front view: (a) Fully depressed. (b) Fully elevated. Ventral view: (c) Fully protracted. (d) Fully retracted. (e) Wing extended. (f) Wing folded. Source: (38, 39).

Colorado et al. (40, 58, 145) put forward a batbot on the basis of the previous work done in Ref. (38). Fig. 2.23 shows a prototype of the bat robot. As shown in Fig. 2.23, a conventional servo was adopted to realize the primary flapping motions while shape memory alloys (SMAs) were used as artificial muscles for morphing wing behaviours (wing retraction and extension). After experimental tests, the frequency of morphing wings can arrive to the maximum value, $2.5 \mathrm{~Hz}$ by carefully control of SMAs (40). In addition, results also suggested that faster contraction of the wings during the upstroke, and slower extension during the downstroke could reduce the energy cost of flapping (58), which is in accordance with the behaviour of real bats.

Table 2.4 describes several significant parameters for morphing-wing FWMAVs, i.e., wing span, total weight, flapping frequency, morphing type and morphing actuator. From the table, we can find that, the research on the bio-inspired morphing concepts mainly concentrates on the passive morphing mechanism based on those already successfully flown ornithopter platforms. Through minor changes in compliance of wing leading edges, passive morphing can be easily achieved in comparison of complex structures of active morphing. There is still a long way to realize the much more complex active morphing like bats' wings, even though the successful 


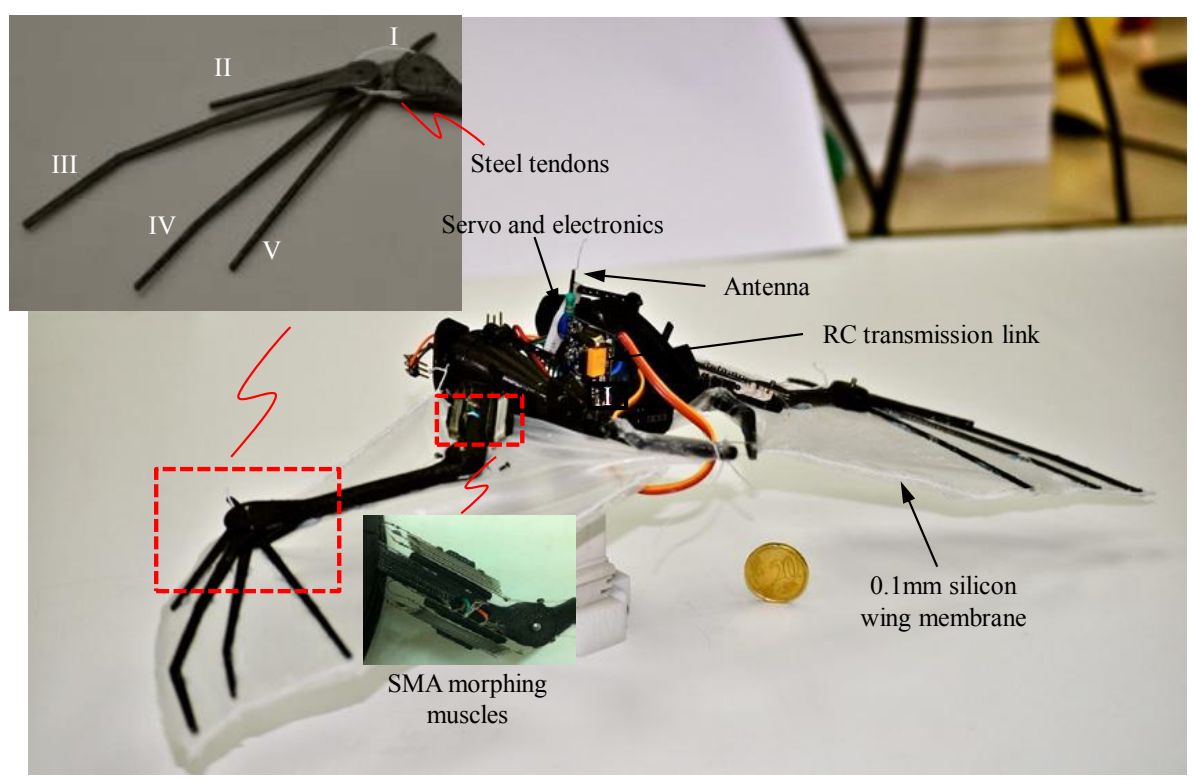

Figure 2.23: A bat-like micro aerial vehicle (Batbot) using NiTi shape memory alloy (SMA) artificial muscles to actuate the morphing wing mechanism. Source: (40).

Table 2.4: Comparison among various bio-inspired morphing concepts for flapping-wing aerial vehicles

\begin{tabular}{|c|c|c|c|c|c|c|}
\hline Group & $\begin{array}{l}\text { Wing } \\
\text { Span } \\
(\mathrm{cm})\end{array}$ & $\begin{array}{l}\text { FWMAV } \\
\text { Weight } \\
(\mathrm{g})\end{array}$ & $\begin{array}{c}\text { Flapping } \\
\text { Frequency } \\
\quad(\mathrm{Hz})\end{array}$ & $\begin{array}{c}\text { Morphing } \\
\text { Type }\end{array}$ & $\begin{array}{l}\text { Morphing } \\
\text { Actuator }\end{array}$ & $\begin{array}{l}\text { Lift-off } \\
\text { or not }\end{array}$ \\
\hline \multicolumn{7}{|l|}{ Univ. of Maryland } \\
\hline FWMAV 28 & 57.2 & 35 & 5 & Passive & Non & Yes \\
\hline \multicolumn{7}{|l|}{ Univ. of Maryland } \\
\hline Modified Park Hawk 29 & 107 & 425 & $4-6$ & Passive & Non & Yes \\
\hline $\begin{array}{l}\text { Stanford Univ. } \\
\text { FWMAV } 33\end{array}$ & 40 & - & $5-17$ & Passive & Non & No \\
\hline \multicolumn{7}{|l|}{ Univ. of Maryland } \\
\hline Modified Park Hawk 30,3132 & 107 & 425 & $4-6$ & Passive & Non & Yes \\
\hline Festo SmartBird (141 142 & 200 & 450 & 2 & Active & Non & Yes \\
\hline BAtMAV $35 \sqrt[36]{35}$ & $25^{\mathrm{a}}$ & - & 12 & Active & SMA & No \\
\hline Batbot 40$] 58$ & 53 & 79 & 2.5 & Active & SMA & No \\
\hline
\end{tabular}

a Estimated from the wingtip trajectory in Ref. (146).

flight of large SmartBird with active variable configuration of wing extremely encourages current researchers. 


\subsection{Conclusion}

From the literature review presented in this chapter, we detected three aspects, summarized below, that need to be investigated.

- Compliant Flapping Mechanism:

As introduced in this chapter, researchers in the FWMAV field have done many remarkable works on the design of compliant flapping mechanisms. However, very few of them have mentioned the details of optimization design. Instead, most researchers seem preferable to display their manufacture procedures and processes. In such situation, we proposed two approaches to optimize primary attributes at compliant joints in the compliant transmission mechanism for flapping. The primary attributes are specified to the torsional stiffness of virtual spring and the initial neutral angular position in the pseudo-rigid model. Compared to other optimization methods proposed (see, e.g. (4, 147)), our methods are suitable for pseudo-rigid models since internal interaction forces do not need to be considered. Furthermore, the elastic potential energy stored in the compliant joints can be easily taken into account. In addition, these methods can be generalized to design and optimize other compliant transmission mechanisms. Those related contents are described in Chapter 3 .

\section{- Flight Control Strategy:}

The control strategies for flight generally can be sort into two categories, linear control and nonlinear control methods. The dynamic models used for linear control almost derive from the simplification and approximation of six-DoF flight models for ordinary standard aircraft. These simplification and approximation get linear models hardly deal with system uncertainties and extra disturbances. A minor perturbation will cause the whole system out of control. Nonlinear models have better performance in this regard due to their minor simplification and approximation or even none in standard dynamic models. However, considering variable unsteady aerodynamic fluids and environmental disturbances, general nonlinear controller may not be qualified. Just as stated in $(64,65)$, a neural-network or learning-type control concept would be a promising option in dealing with such situation. As a result, type-1 fuzzy neural networks have been investigated for control schemes for flapping wing micro-air vehicles (see, e.g., Guo et al. (126), Cheng et al. (148) ). In this thesis, we will extend the type-1 fuzzy neural networks into type-2 
fuzzy neural networks, which have been demonstrated that type-2 fuzzy neural networks have much stronger capabilities in coping with uncertainties that type-1 counterparts. Moreover, a feedback-error-learning control frame is employed for the attitude control of a FWMAV, in which a type-2 fuzzy neural network works in parallel with a ordinary proportional derivative controller. More detailed information will be introduced in Chapter 4.

- Morphing-wing Structure:

Currently, the ways used for morphing wings mainly include two aspects. Just as mentioned above, one is passively morphing while the other is actively morphing. The research of passive morphing-wing structures mainly focuses on changing the compliance of leading edge spars for wings. While flapping, wings can negatively response to aerodynamic forces and torques caused by flapping motions through passively changing wings' configuration. A remarkable feature of the structures is that people are out of the control loop of wings. Under the conditions with variable and unsteady aerodynamic fluids, wings symmetrically equipped on the fuselage may produce asymmetrical lift and thrust forces, which will threaten the stability of flight. In contrast, the start point of active morphing structures is to let people stay in the control loop and thereby timely change wing shapes as desired. At present, the investigations of actively morphing wings are primarily inspired by bat wings. Bat wings own unique muscles that allow them fold, expand and camber their wings during flight. Changing the wing profile can improve the generation of lift forces and reduction of drag during the wingstroke. In addition, their high body-to-wing has ratio and high wing dexterity make aggressive maneuvers modulating solely wing inertial possible. In this thesis, new improvements on the morphing-wing structures are made on the basis of the work in (40, 58) and meanwhile experimental tests are carried out to check the influences of morphing wings in terms of lift, thrust and maneuverability. The whole process of design, fabrication and experiments on new morphing structures will be shown in Chapter 5 


\section{3 \\ Design and Optimization of Compliant Transmission Mechanism}

\subsection{Introduction}

In recent decades, natural flying insects, birds and bats are becoming attractive models to imitate in design and development of flapping wing micro air vehicles (FWMAVs) as they are capable of excellent maneuverabilities. In such case, designing a proper and effective flapping mechanism plays a significant role in fabricating a FWMAV. During every flapping cycle, wings have to experience an acceleration phase from rest at the beginning of a stroke and a deceleration phase to stop at the end of the stroke and then the process reverses in the next stroke, which can easily get the driving motor shocked even broken with an effect of aerodynamic forces produced by flapping wings. This unworthy shock needs to be minimized in the design of flapping mechanism as possible for making sure that the motor safely run and thereby generate stable lift and thrust forces required.

Inherently, the introduction of coil springs can tolerate these unexpected shocks (149) in those bio-inspired robots. Research results from both Madangopal et al.(4) and Baek et al. (무) show that the introduction of linear coil springs can minimize the required input peak torque or input power. The use of linear coil springs which are directly connected to DC motors in driving flapping-flight prototypes can also be found in the literature (7, 8). However, additional springs not only bring unneeded payloads, but also do not reduce joint frictions that cause 
energy losses (12). Compared with above spring mechanisms, compliant mechanisms directly combine functions of mechanical parts with elastic energy-stored components together (87), which lets them have advantages compared to rigid-body mechanisms, such as lower wear, friction, and backlash (150, 151), and are compatibility with microelectromechanical systems (MEMS) processing techniques(15).

Compliant mechanisms for FWMAVs have already been employed in few prototypes (see, e.g., (9, 10, 13, 88)). However, almost none of such prototypes take into account the influences of the characteristics of compliant joints or hinges (e.g., the stiffness of virtual spring and the neutral angular positions) on the final input peak torque. In addition, most works lack an exhaustive theoretical analysis. In this chapter, we present an elaborate theoretical analysis on the transmission mechanism and the effects of the compliant attributes on the final peak input torque by using the principle of virtual work and rigid-body dynamics, respectively.

In the following, we present two compliant flapping mechanisms. The first one is proposed based on the work in (9, 10), which has been used as a flapping transmission mechanism for preliminary morphing-wing experiments in Chapter 5. After that, an improved design on basis of the experimental experience gained with the first mechanism is put forward. The detail description is shown in the Section 3.3 of this chapter.

\subsection{First-version Compliant Transmission Mechanism}

As described in Section 2.2, compliant mechanisms are multifunctional structures which combine functions of mechanical parts with elastic and energy-stored components together (87). The stored energies can be recycled and prevent full energies transfer from input port to out port. Therefore, a compliant transmission mechanism based on the work in (9, 10) was proposed in Fig. 3.1. Fig. 3.1 shows a snapshot of the compliant mechanism used in our morphing-wing robot, shot during tests using a high-speed camera. A crank-and-rocker mechanism was adopted for realising flapping-wing motion. Link OA is a crank, which is connected to the rocker, link $\mathrm{AB}$. The rotation of the motor is transformed to up-and-down motions of flapping-wing arms $\mathrm{BC}$ and $\mathrm{BE}$ along the central vertical groove between two wing supporters $\mathrm{CD}$ and $\mathrm{EF}$. Note that the wing supporters $\mathrm{CD}$ and $\mathrm{EF}$ are compliant beams, which flapping-wing arms $\mathrm{BC}$ and $\mathrm{BE}$ are mounted on, respectively. They allow for the displacement at pivoting points $\mathrm{C}$ and $\mathrm{E}$. In order to eliminate out-of-plane motions during flight, a biplanar design like the one proposed in (9) was taken into account. This is shown in Fig. 3.2 . 


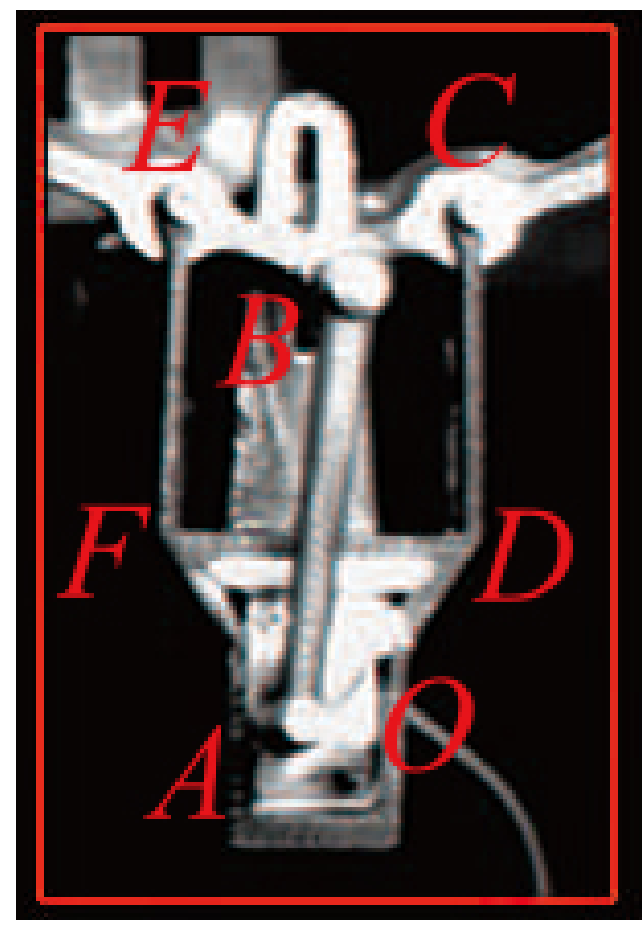

Figure 3.1: Detail of the compliant actuation mechanism for a morphing-wing flying robot

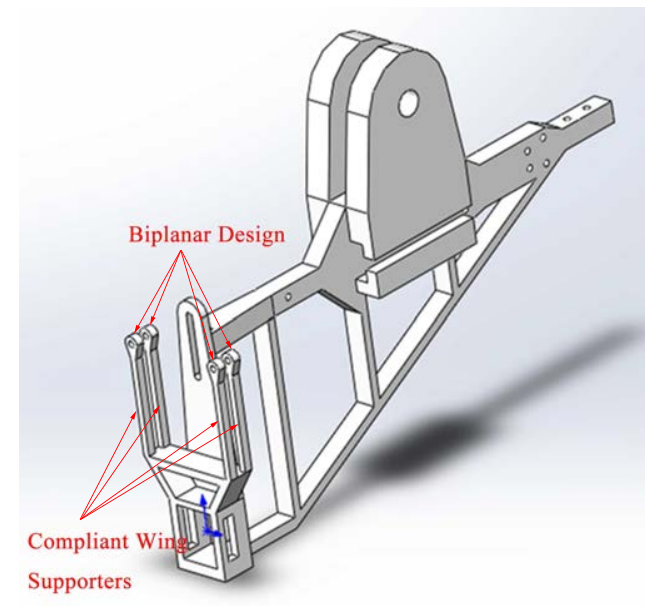

Figure 3.2: Main body of the morphing-wing flying robot

Here, the pseudo-rigid-body (PRB) theory is used to analyse compliant mechanisms (152, 153), since it can directly use current available rigid-body approaches instead of large-deflection non-linear analysis of compliant mechanisms (154). Fig. 3.3 shows a half PRB model of the symmetry driving mechanism (Note the correspondence of points A, B, C, D, O of Fig. 3.1). 


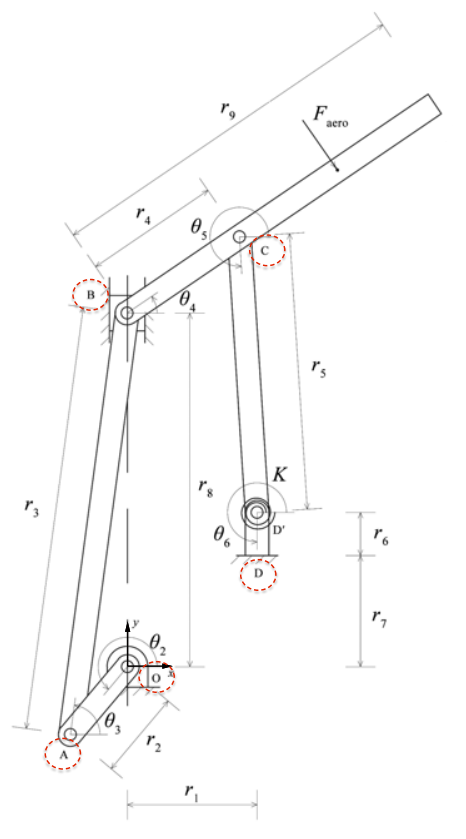

Figure 3.3: Pseudo-rigid-body model of compliant driving mechanism

Table 3.1: Key Dimensions of Compliant Actuator Mechanism

\begin{tabular}{c||c||c||c}
\hline Parameters & Scales $[\mathrm{mm}]$ & Parameters & Scales $[\mathrm{mm}]$ \\
\hline \hline$r_{1}$ & 15 & $r_{5}$ & 22.31 \\
\hline$r_{2}$ & 6 & $r_{6}$ & 3.94 \\
\hline$r_{3}$ & 32.75 & $r_{7}$ & 9 \\
\hline$r_{4}$ & 15 & $r_{9}$ & 240 \\
\hline$l_{C D}$ & 26.25 & $\gamma$ & 0.85 \\
\hline
\end{tabular}

The compliant part was approximated as rigid link with length $r_{5}$ and a torsion spring with constant $K$ at the pin joint. The key dimensions of the drive mechanism are shown in Table 3.1. In this mechanism we have that

$$
K=\gamma K_{s} \frac{E I}{l_{C D}}
$$

where $\gamma$ is the characteristic radius factor, $K_{s}$ is the stiffness coefficient, $E$ is Young's elastic modulus of material, $I$ is the cross-sectional moment of inertia of the compliant wing supporter $\mathrm{CD}$ and $l_{C D}$ is its length. 


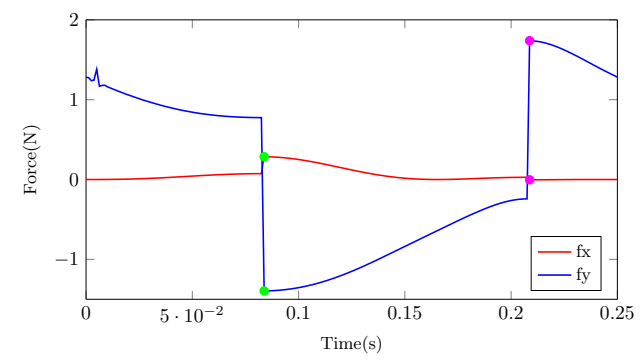

Figure 3.4: Forces in $x$ and $y$ directions during one circle flapping at joint C. The $x$ and $y$ values of the green points were $0.2859 \mathrm{~N}$ and $-1.3957 \mathrm{~N}$ while those of the magenta ones along $x$ and $y$ axises were $-0.0034 \mathrm{~N}$ and $1.7393 \mathrm{~N}$,respectively.

\subsubsection{Force Estimation acting on Wing Supporters}

After the dimensional parameters of the driving system had been chosen, structural analysis of the mechanism was taken into consideration for ensuring the whole driving system was robust enough. In this section, we analyse the compliant wing supporters as they are crucial for realising the flapping functions required. To do so, we built the PRB model in MSC Adams/View 2013 software and used it to assess the forces acting on the compliant wing supporters and therefore carried out the structural analysis. The aerodynamic forces generated by wings was set to act in the middle of wing arms and norm to them. The directions of aerodynamic forces were opposite to the direction of wings' actions, i.e., the directions of aerodynamic forces were perpendicular to wing arms downwards during upstroke and vice versa. The masses of moving elements (e.g. the crank, rocker) were very small so that their inertial forces were ignored.

We simulated the model at the condition of static equilibrium. The rotation speed of DC motor was set to $240 \mathrm{rpm}$. The aerodynamic force $F_{a e r o}=a * \operatorname{sgn}\left(v_{f w}\right)$ and $v_{f w}$ is flapping wing speed. The materials of all components used for simulation were acrylonitrile butadiene styrene $(\mathrm{ABS})$, with density $=1040 \mathrm{~kg} / \mathrm{m}^{3}$, Poisson's ratio $=0.3$ and Young's Modulus $=2.3$ GPa. Because the four compliant wing supporters are the same, we just recorded and plotted the reaction forces at point $\mathrm{C}$ (see Fig. 3.1 and 3.3) with the resolution of $0.25 \mathrm{~s}$. The detail forces produced by wings in both $\mathrm{x}$ and $\mathrm{y}$ directions during one flapping circle were displayed in Fig. 3.4. The green and magenta points in the figure represent the $x$ and $y$ direction forces which could cause wing supporters to break. 


\subsubsection{Optimization Design and Stress Analysis of Wing Supporters}

In this subsection, we deal with the rectangular cross-section optimization of wing supporters for the purpose of satisfying physical and stress requirements and minimizing the mass of wing supporters as well. The cross-section optimization problem was formulated as follows:

$$
\begin{gathered}
\text { minimize } \quad m_{w s}=w d l_{C D} \rho \\
\text { subject to } \quad w \in\left[w_{l}, w_{u}\right] \\
d \in\left[d_{l}, d_{u}\right] \\
\frac{\left|f_{y}\right|}{w d}+\frac{|M| d}{2 I} \leq \frac{\sigma_{\max }}{n}
\end{gathered}
$$

where $w$ and $d$ are the width and depth of the rectangular cross-section, $w_{l}, w_{u}, d_{l}, d_{u}$ are the lower and upper limits of the width and depth, respectively. $M$ is the torque caused by $x$ direction force $f_{x}, M=f_{x} l_{C D} . f_{y}$ is the force acting in $y$ direction. $I$ is the cross-sectional moment of inertia of the compliant wing supporter, $I=\frac{1}{12} w d^{3} . \sigma_{\max }$ is the maximum allowable tensile stress of $\mathrm{ABS}$ and $n$ is a safety factor.

This is a single-objective constrained optimization problem. Here, we used a hybrid multiswarm particle swarm optimization (HMPSO) algorithm (157) to solve the optimization problem. HMPSO adopts a parallel search operator in which the current swarm is partitioned into several subswarms and particle swarm optimization (PSO) is severed as the search engine for each sub-swarm. Moreover, in order to explore more promising regions of the search space, differential evolution (DE) is incorporated to improve the personal best of each particle. In detail, initially, the swarm $P_{0}$ of size $N$ is randomly and uniformly selected between the lower and upper bounds defined for each variable. At each generation, the swarm is first split into several sub-swarms and each swarm evolves in parallel. After that, the personal best of each particle is updated by DE. The Pseudo code of HMPSO is depicted in Algorithm 1 .

$$
\begin{gathered}
v_{i, j}^{t+1}=\|\operatorname{randn}\|\left(\text { pbest }_{i, j}^{t}-x_{i, j}^{t}\right)+\|\operatorname{Randn}\|\left(\text { lbest }_{i, j}^{t}-x_{i, j}^{t}\right) \\
x_{i, j}^{t+1}=x_{i, j}^{t}+v_{i, j}^{t+1}
\end{gathered}
$$

where $\overrightarrow{l b e s t}_{i}^{t}=\left(l b e s t_{i, 1}^{t}, \ldots, l b e s t_{i, n}^{t}\right)$ is the best position achieved with its neighbours. $\left(p b e s t_{i, j}^{t}-\right.$ $\left.x_{i, j}^{t}\right)$ and $\left(\right.$ lbest $\left.t_{i, j}^{t}-x_{i, j}^{t}\right)$ are a personal term and a local term, respectively. $\|r a n d n\|$ and 


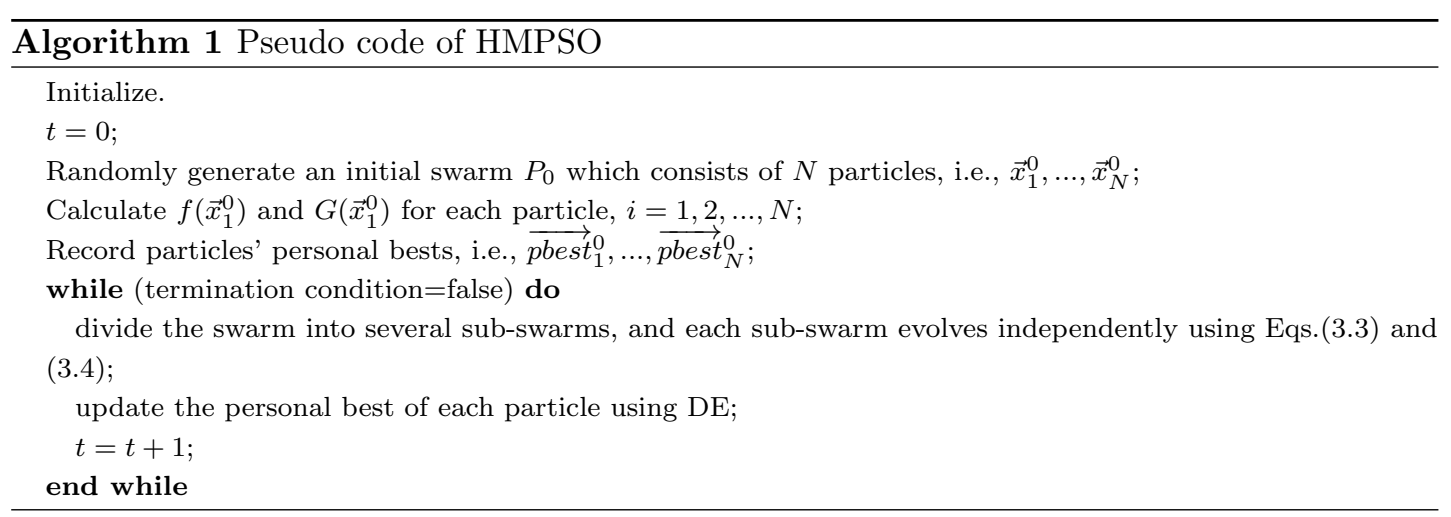

Table 3.2: Input Parameters for the Cross-section Optimization of wing supporters

\begin{tabular}{c||c||c||c}
\hline Parameters & Scales & Parameters & Scales \\
\hline \hline$w_{l}$ & $0.002 \mathrm{~m}$ & $w_{u}$ & $0.005 \mathrm{~m}$ \\
\hline$d_{l}$ & $0.001 \mathrm{~m}$ & $d_{u}$ & $0.005 \mathrm{~m}$ \\
\hline$f_{x}(1)$ & $0.2859 \mathrm{~N}$ & $f_{y}(1)$ & $-1.3957 \mathrm{~N}$ \\
\hline$f_{x}(2)$ & $-0.0034 \mathrm{~N}$ & $f_{y}(2)$ & $1.7393 \mathrm{~N}$ \\
\hline$\sigma_{\max }$ & $30 \mathrm{MPa}$ & $n$ & 3 \\
\hline
\end{tabular}

$\|$ Randn\| are two stochastic coefficients, which are generated by the absolute value of the Gaussian probability distribution with zero mean and unit variance, i.e., $\operatorname{abs}(\mathrm{N}(0,1))$.

The parameters used for the optimization are shown in Table 3.2 and the procedure was executed in MATLAB 2014a with a population size of 60 and was run for 250 generations. The optimisation included two cases: (i) $f_{x}=0.2859 \mathrm{~N}, f_{y}=-1.3957 \mathrm{~N}$; (ii) $f_{x}=-0.0034 \mathrm{~N}$, $f_{y}=1.7393 \mathrm{~N}$. In case I, $w$ and $d$ were calculated to be $0.0020 \mathrm{~m}$ and $0.0014 \mathrm{~m}$ while $w$ and $d$ equalled $0.0020 \mathrm{~m}$ and $0.0010 \mathrm{~m}$ in case II, respectively. Therefore, the width and depth of wing supporters were set $2 \mathrm{~mm}$ and $1.5 \mathrm{~mm}$, respectively. To analyse the Von Mises stress of wing supporters we used SolidWorks CAD software to perform the finite element analysis. Due to the biplanar design, the forces acting on a single wing supporter were only a half of the values at green and magenta points in Fig. 3.4. The analysis results are shown in Fig. 3.5 and Fig. 3.6. This analysis demonstrated that the stresses resulting from the frame loads were safe and it was also useful to find the areas with high stresses and improve the mechanical design of the robot. 


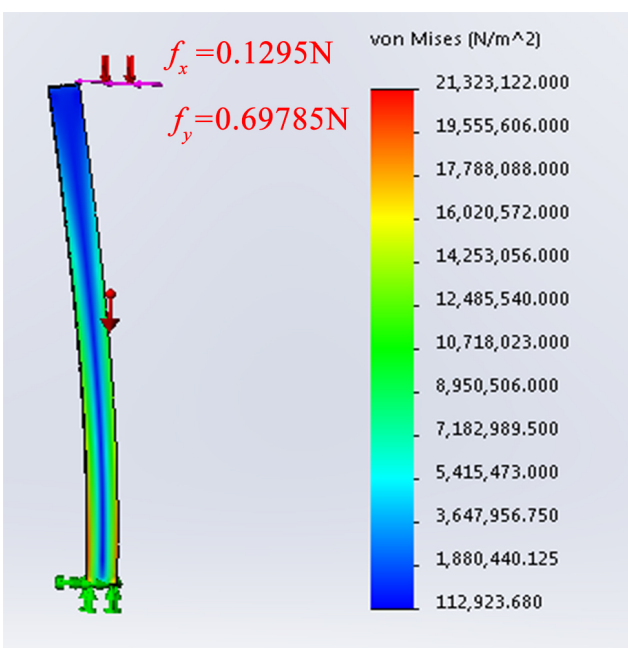

Figure 3.5: Von Mises stress with a horizontal pull force $0.1295 \mathrm{~N}$ and vertical push force 0.69785 $\mathrm{N}$

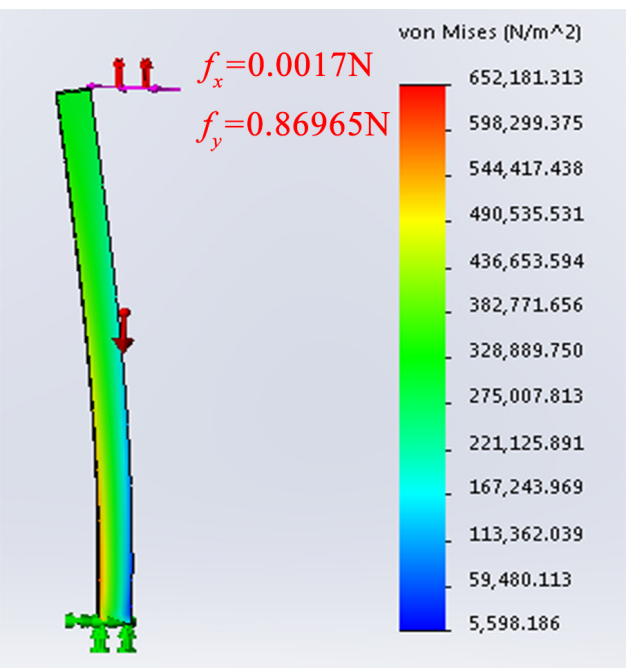

Figure 3.6: Von Mises stress with a horizontal pull $0.0017 \mathrm{~N}$ and vertical pull force $0.0125 \mathrm{~N}$

\subsection{A Second Improved Compliant Transmission Mecha- nism and Its Kinematics}

During the process of experiments carried out with the first-version flapping mechanism, we found that the compliant design could not reduce the sharp peak shock obviously, since the peak input torque is only slightly reduced (see Fig. 3.12 , right), compared to a rigid-body mechanism. It could be further lowered by adding compliant joints at shoulders. Therefore, we present an 


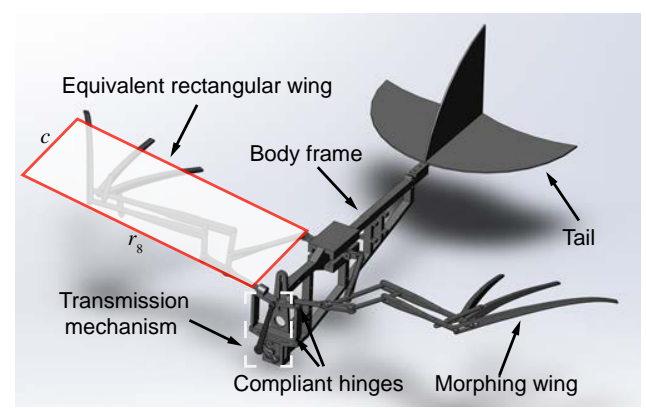

Figure 3.7: A closeup of the CAD model for a bat-inspired flapping-wing aerial vehicle.

Table 3.3: Component Dimensions of the Compliant Transmission Mechanism (see also Fig. 3.8 .

\begin{tabular}{ccc}
\hline \hline Link & Symbol & Scale \\
\hline OA & $r_{1}$ & $6.0 \mathrm{~mm}$ \\
AB & $r_{2}$ & $32.8 \mathrm{~mm}$ \\
BC & $r_{3}$ & $15.0 \mathrm{~mm}$ \\
CD & $r_{4}$ & $22.4 \mathrm{~mm}$ \\
OB & $r_{5}$ & - \\
Horizontal Distance OD & $r_{6}$ & $15.0 \mathrm{~mm}$ \\
Vertical Distance OD & $r_{7}$ & $13.0 \mathrm{~mm}$ \\
CE & $r_{8}$ & $235.0 \mathrm{~mm}$ \\
\hline \hline
\end{tabular}

improved flapping transmission mechanism with small-length lightweight compliant hinges and then perform kinematic analysis of it. The proposed compliant hinges are equivalent as revolute joints with virtual springs based on the PRB theory proposed in (49). A CAD cutaway of main airframes is shown in Fig. 3.7. The robot consists of morphing wings, a tail, a body frame and a compliant transmission mechanism. A small DC motor running in anti-clockwise direction is employed to drive a compliant transmission mechanism to realize flapping motions. The mass of wing frames is assumed to be uniformly distributed along the length of the wing spar. Since the robot has a symmetrical structure, only half of the structure is analysed here. The schematic closeup of the half transmission mechanism is shown in Fig. 3.8 (a) and its corresponding PRB model is presented in Fig. 3.8(b). The dimensions of the primary frames are listed in the Table 3.3. 
(a)

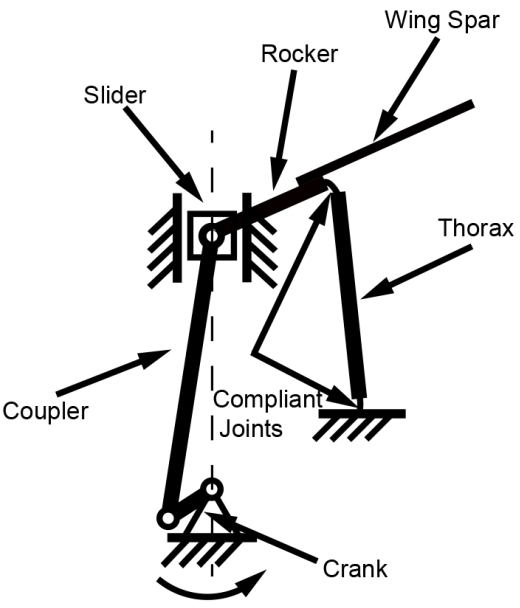

(b)

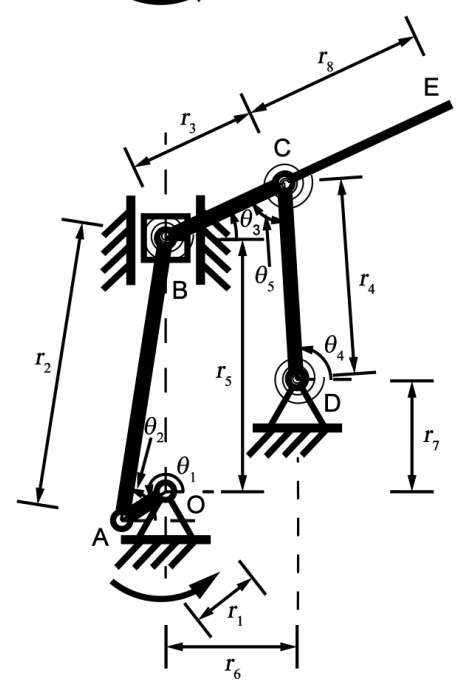

Figure 3.8: (a) A schematic view of the compliant transmission mechanism during upstroke. (b) Pseudo-rigid-body model of the compliant transmission mechanism.

\subsubsection{Kinematic Analysis}

Considering the vector loops O-A-B-C-D-O and O-A-B-O shown in Fig. 3.8(b), the loop closure equations in form of complex members are:

$$
\begin{gathered}
r_{1} e^{j \theta_{1}}+r_{2} e^{j \theta_{2}}-r_{3} e^{j\left(\pi+\theta_{3}\right)}-r_{4} e^{j \theta_{4}}-r_{6} e^{j 0}-r_{7} e^{j \frac{\pi}{2}}=0 \\
r_{1} e^{j \theta_{1}}+r_{2} e^{j \theta_{2}}-r_{5} e^{j \frac{\pi}{2}}=0
\end{gathered}
$$




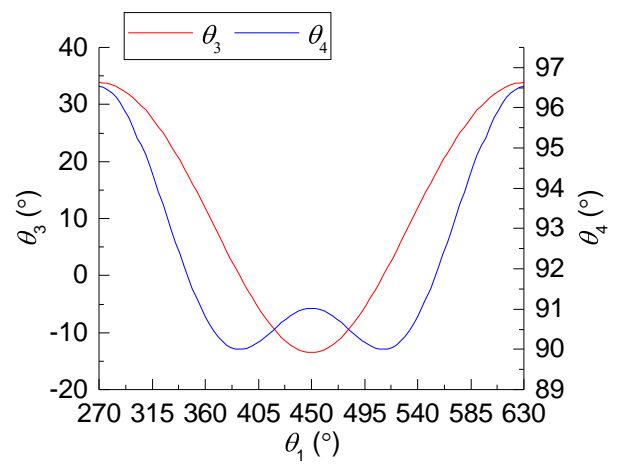

Figure 3.9: Variations on the flapping angle of a wing and the torsional angle at compliant joint D during a flapping cycle.

where $r_{i}(i=1,2, \ldots, 6,7)$ are lengths of links and $\theta_{j}(j=1,2, \ldots, 4)$ are angular positions of the corresponding links.

Since this driving mechanism has only one degree of freedom (DoF), i.e., the angular position of the crank $\theta_{1}$, thus the rest angular positions $\theta_{2}, \theta_{3}, \theta_{4}$ can be treated as functions in terms of $\theta_{1}$. Here we define $\dot{\theta}_{i}=\frac{\mathrm{d} \theta_{i}}{\mathrm{~d} \theta_{1}}, \ddot{\theta}_{i}=\frac{\mathrm{d} \dot{\theta}_{i}}{\mathrm{~d} \theta_{1}}(i=1,2,3,4)$. Note that $\dot{\theta}_{1}$ equals 1 and $\ddot{\theta}_{1}$ equals 0 , since the motor is considered to be rotating at constant speed, whose value is $600 \mathrm{rpm}$. Considering the velocity and acceleration of the angular position $\theta_{i}(i=1,2,3,4)$ with respect to time $t, \omega_{i}$ and $\alpha_{i}$, which are defined as $\omega_{i}=\frac{\mathrm{d} \theta_{i}}{\mathrm{~d} t}, \alpha_{i}=\frac{\mathrm{d} \omega_{i}}{\mathrm{~d} t}$, therefore, the velocity $\omega_{i}$ and acceleration $\alpha_{i}$ can be expressed as $\omega_{i}=\dot{\theta}_{i} \omega_{1}$ and $\alpha_{i}=\ddot{\theta}_{i} \omega_{1}^{2}+\dot{\theta}_{i} \alpha_{1}$.

When the initial values of angular positions $\theta_{1}, \theta_{2}, \theta_{3}, \theta_{4}$ are known, their corresponding values in a whole flapping cycle can be solved after separating real and imaginary components of equations (3.5) (3.6) and combining them together. Fig. 3.9 shows the flapping angle $\theta_{3}$ and the angular variety $\theta_{4}$ of the thorax during a flapping cycle. Similarly, angular velocities $\dot{\theta}_{i}$ and $\omega_{i}$ and accelerations $\alpha_{i}(i=2,3,4)$ of the links can also be computed according to the first and second order derivatives of the two position equations $(3.5)$ and $(3.6)$.

\subsection{Aerodynamic Torque}

Aerodynamic torques generated by flapping wings play a very important role in the stable flights of FWMAVs. In this section, we analyse the aerodynamic torque produced by wing motions in a cycle. To this purpose, an aerodynamic model based on the blade element theory (156) was employed. For simplifying the calculation, the irregular shape of a wing is approximated with a rectangle, whose length and width equal the length of the wing spar $r_{8}$ and the average 
wing chord $c$, respectively. Note that the average chord $c$ is computed according to wings' equivalent surface area. Here $c$ equals $10.25 \mathrm{~cm}$. The wing is assumed to be rigid, i.e., without any twist and bend along the wing chord and the leading edge. The mass of the wing membrane is considered to be negligible. Therefore, the normal force produced by a single blade can be expressed according to the theory in $(\underline{156})$ as follows:

$$
d F_{a}=-\frac{1}{2} \rho C_{1}|V(r, t)|^{2} \operatorname{sgn}(V(r, t)) c d r
$$

where $V(r, t)$ is the absolute velocity of an element, whose direction is contrary to the motion of the wing, $\rho$ is the air density, $1.23 \mathrm{~kg} / \mathrm{m}^{3}$, and $C_{1}$ is the normal force coefficient of the blade(158). Note that $V(r, t)$ consists of two parts: translational velocity $V(r, t)_{T}$ and rotational velocity of wings $V(r, t)_{R}$ (see Fig. 3.10). Thus, $V(r, t)=V(r, t)_{T}+V(r, t)_{R}$ and $|V(r, t)|=$ $\left|V(r, t)_{T}\right|+\left|V(r, t)_{R}\right|$. Be ware that $\left|V(r, t)_{T}\right|=r_{4} \omega_{4} \cos \left(\theta_{5}\right),\left|V(r, t)_{R}\right|=r \omega_{3}$. Here, $\theta_{5}$ is the intersection angle between the rocker $r_{3}$ and the thorax $r_{4}$ as shown in Fig. 3.10. As the value of the translation velocity $V(r, t)_{T}$ is relatively small, its effect of wings' movements is also relatively few. Therefore, $V(r, t)$ and $V(r, t)_{R}$ can be treated as they have the same values, i.e., $V(r, t)=V(r, t)_{R}, \operatorname{sgn}(V(r, t))=\operatorname{sgn}\left(V(r, t)_{R}\right)$. Since $V(r, t)_{R}$ is positively proportional to $\omega_{3}$, $\operatorname{sgn}(V(r, t))$ can be described in terms of $\operatorname{sgn}\left(\omega_{3}\right)$ as $\operatorname{sgn}(V(r, t))=\operatorname{sgn}\left(\omega_{3}\right)$.

The instantaneous aerodynamic torque $\tau_{a}$ can be expressed by the instantaneous force $d F_{a}$ times the distance $r$ along the wing spar,

$$
d \tau_{a}=-\frac{1}{2} \rho C_{1} r^{3} \omega_{3}^{2} \operatorname{sgn}\left(\omega_{3}\right) c d r
$$

\subsection{Input Torque Calculation Based on the Principle of Virtual Work}

After the kinematic analysis of the mechanism and calculation of aerodynamic torque produced by a wing, we will derive the input torque required from the motor based on the principle of virtual work. This method is well suitable for PRB models since it treats the system as a whole, and internal interaction forces are not needed to be considered. Furthermore, elastic potential energy stored in the compliant joints can be easily taken into account. Here, the transmission system is supposed to work in a plane. The masses of links (except the wing spar), inertial forces and frictions at joints are negligible in comparison with external aerodynamic forces and applied torque from the motor. 


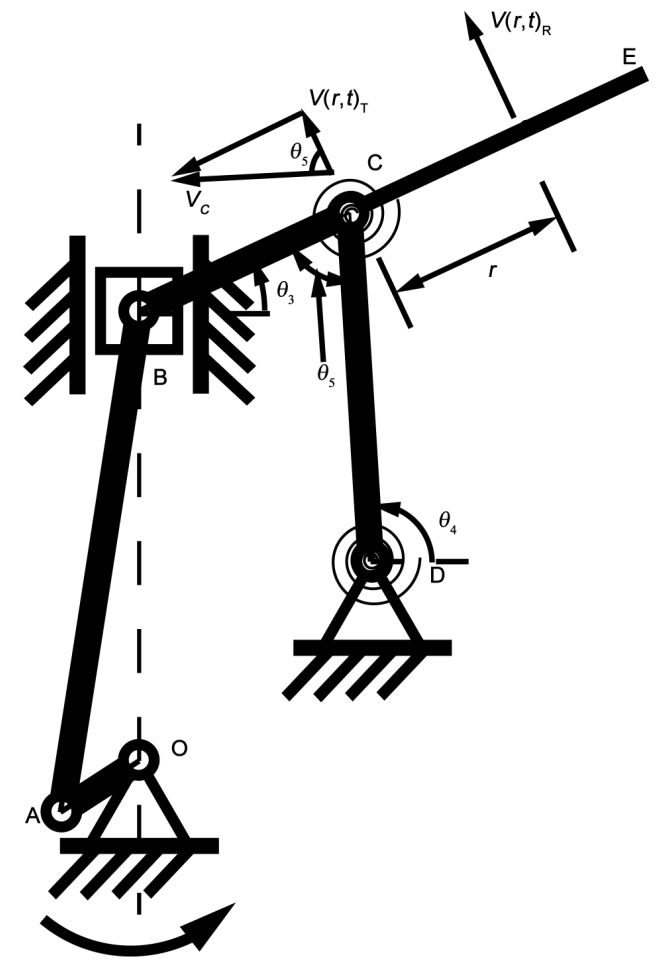

Figure 3.10: The sketch of the velocity analysis on the wing.

According to the PRB model shown in the Fig. 3.8, the generalized coordinate is chosen to be the angular displacement of the crank $\theta_{1}$ and its virtual displacement is $\delta \theta_{1}$. The total virtual work $\delta W$ includes three parts: the work done by the motor through external input torque $\tau_{m}$, $\delta W_{m}$, the work caused by inertial and aerodynamic torques of the wing, $\delta W_{w}=\delta W_{\text {inert }}+\delta W_{w}$, and the work stored by the equivalent springs at compliant joints, $\delta W_{s}$. Thus,

$$
\delta W=\delta W_{m}-\delta W_{w}-\delta W_{s}
$$

Since the mass of wing membrane is too small to be neglected, the mass of wing approximately equals the mass of wing spar. As shown in the Fig. 3.8, the motion of wing spar can be treated as a synthesis of a axis-fixed rotational movement around the joint $\mathrm{C}$ and a translational movement around the joint D. Therefore, the virtual work produced by the inertial torque of the rectangular wing is simply given as

$$
\delta W_{\text {inert }}=\frac{1}{3} m_{8} r_{8}^{2} \alpha_{3} \delta \theta_{3}+m_{8} r_{4}^{2} \alpha_{4} \delta \theta_{4}
$$


where $m_{8}$ is the mass of the wing spar. $\frac{1}{3} m_{8} r_{8}^{2}$ and $m_{8} r_{4}^{2}$ are the inertias of the wing with respect to the joint $\mathrm{C}$ and $\mathrm{D}$, respectively. In addition, the virtual work $\delta W_{a}$ caused by aerodynamic forces is given as $\delta W_{a}=-\tau_{a} \delta \theta_{3}$, therefore,

$$
\delta W_{w}=\frac{1}{3} m_{8} r_{8}^{2} \alpha_{3} \delta \theta_{3}+m_{8} r_{4}^{2} \alpha_{4} \delta \theta_{4}-\tau_{a} \delta \theta_{3}
$$

The virtual work at compliant joints is $\delta W_{s}=-\sum_{i=1}^{2} T_{i} \delta \psi_{i}$, where $T_{i}=-K s_{i} \psi_{i},(i=1,2)$, $K s_{i}$ is the torsional stiffness constant at the $i$ th $(i=1,2)$ compliant joint. Note that joints $\mathrm{C}, \mathrm{D}$ are defined as the 1 st and 2 nd joints, respectively. $\psi_{i}$ is the angular variation at the $i$ th joint, which is expressed as $\psi_{1}=\theta_{4}-\theta_{4,0}, \psi_{2}=\theta_{5}-\theta_{5,0}$ and those associated with $\delta \psi_{i}$ are: $\delta \psi_{1}=\delta \theta_{4}, \delta \psi_{2}=\delta \theta_{5}$. Here, $\theta_{j}, \theta_{j, 0}(j=4,5)$ are the angular position and neutral angular position at the two compliant joints, respectively. Be ware that $\theta_{5}=\theta_{4}-\theta_{3}, \theta_{5,0}=\theta_{4,0}-\theta_{3,0}$, therefore, $\delta \theta_{5}=\delta \theta_{4}-\delta \theta_{3}, \delta \psi_{2}=\delta \theta_{5}=\delta \theta_{4}-\delta \theta_{3}$. The virtual work stored at compliant joints is

$$
\delta W_{s}=K s_{1}\left(\theta_{4}-\theta_{40}\right) \delta \theta_{4}+K s_{2}\left[\left(\theta_{4}-\theta_{3}\right)-\left(\theta_{4,0}-\theta_{3,0}\right)\right]\left(\delta \theta_{4}-\delta \theta_{3}\right)
$$

According to the principle of the virtual work, the total virtual work is set to equal to zero. The equation 3.9 can be solved in terms of the unknown input torque $\tau_{m}$,

$$
\tau_{m}=\frac{1}{3} m_{8} r_{8}^{2} \alpha_{3} \dot{\theta}_{3}+m_{8} r_{4}^{2} \alpha_{4} \dot{\theta}_{4}-\tau_{a} \dot{\theta}_{3}+K s_{1}\left(\theta_{4}-\theta_{40}\right) \dot{\theta}_{4}+K s_{2}\left[\left(\theta_{4}-\theta_{3}\right)-\left(\theta_{4,0}-\theta_{3,0}\right)\right]\left(\dot{\theta}_{4}-\dot{\theta}_{3}\right)
$$

\subsection{Input Torque Calculation Based on Rigid-body Dy- namics}

In the previous section, we have already calculated the input torque needed based on the principle of virtue work. In this section, we try to solve the same problem from a perspective of energy. First of all, we will focus on kinetic and potential energies of the mechanism. Based on the energy equations, a rigid-body dynamic model is built for clearly understanding the requirement of input torque of motor during a cycle. 


\subsection{Input Torque Calculation Based on Rigid-body Dynamics}

For the crank, it rotates around a fixed end $\mathrm{O}$ with a constant speed so it only has a rotational kinetic energy, which is given as

$$
K_{1}=\frac{1}{2} J_{1} \omega_{1}^{2}
$$

where $J_{1}$ is its mass moment of inertia about the end $\mathrm{O}$. As for the coupler and rocker, they possess both translational and rotational movements around their centers of mass (CoMs), therefore, their corresponding kinetic energies $\left(K_{i}\right)$ consist of two parts, i.e., translational kinematic energy $\left(K_{T_{i}}\right)$ and rotational kinematic energy $\left(K_{R_{i}}\right)$,

$$
K_{i}=K_{T_{i}}+K_{R_{i}}
$$

where

$$
K_{T i}=\frac{1}{2} m_{i} V_{c i}^{2}, K_{R i}=\frac{1}{2} J_{i} \omega_{i}^{2},
$$

Here, $m_{i}(i=2,3)$ is the mass of link $i(i=2,3), V_{c i}(i=2,3)$ is the absolute velocity at the CoM, $J_{i}(i=2,3)$ is the mass moment of inertia about the corresponding CoM. Similarly, we can also calculate translational and rotational kinetic energies of the wing spar:

$$
K_{T 8}=\frac{1}{2} m_{8} V_{C}^{2}, K_{R 8}=\frac{1}{2} J_{8} \omega_{3}^{2}
$$

where $m_{8}$ is the mass of link $8, V_{C}$ is the absolute velocity at joint C, where $V_{C}=r_{4} \omega_{4}, J_{8}$ is the mass moment of inertia about the joint $\mathrm{C}, \omega_{3}$ is the corresponding angular velocity of the rocker which wing spar connects to.

As for compliant wing supporters, their equivalent kinetic energy are given as follows according to the method proposed in (154),

$$
K_{4}=\frac{1}{8} m_{4} r_{4}^{2} \omega_{4}^{2}+\frac{1}{2} J_{4} \omega_{4}^{2}
$$

where $m_{4}$ is the mass of wing supporter $4, V_{C}$ is the absolute velocity at joint $\mathrm{C}, J_{4}$ is the mass moment of inertia about joint D.

We assumed that the robot is inertially fixed on the earth. In this case, no potential energy produces by gravity. The potential energy only contains potential energies generated 


\subsection{Input Torque Calculation Based on Rigid-body Dynamics}

by torsional springs. The potential energy stored in every spring can be expressed in general expression as,

$$
P_{i}=-\frac{1}{2} K s_{i} \psi_{i}^{2}
$$

where $K s_{i}$ is the torsional stiffness constant at the $i$ th $(i=1,2)$ compliant joint. Here, joints C, D are also defined as the 1 st and 2 nd joints, respectively. $\psi_{i}$ is the angular variation at the $i$ th joint. Their expressions are given as in previous Section 3.5.

The total kinetic energy $K$ and potential energy $P$ of the entire system are the sum of the kinetic energies of all the links and and potential energies of all equivalent springs, respectively,

$$
K=\sum K_{i}, P=\sum P_{i}
$$

Then, Lagrange's equation of the whole system is

$$
L=K-P
$$

As stated above, this system has only one degree of freedom $\theta_{1}$, therefore, the Lagrange's equation of motion with respect to $\theta_{1}$ is

$$
\frac{\mathrm{d}}{\mathrm{d} t}\left[\frac{\partial L}{\partial \omega_{1}}\right]-\frac{\partial L}{\partial \theta_{1}}=\tau_{s}
$$

where $\tau_{s}$ is the input torque of motor with the effect of inertial moment of the mechanical system and torsional springs.

Suppose the mass of link $r_{i}(i=1,2,3,4)$ is too small to be ignored, therefore, $K_{i}=0$ $(i=1,2,3,4)$. In such case, the Lagrange's equation is

$$
L=\frac{1}{2} m_{8} V_{C}^{2}+\frac{1}{2} J_{8} \omega_{3}^{2}+\frac{1}{2} K s_{1} \psi_{1}^{2}+\frac{1}{2} K s_{2} \psi_{2}^{2}
$$

Substituting Eq. 3.23 into Eq. 3.22, we can obtain

$$
\tau_{s}=\frac{1}{3} m_{8} r_{8}^{2} \alpha_{3} \dot{\theta}_{3}+m_{8} r_{4}^{2} \alpha_{4} \dot{\theta}_{4}+K s_{1}\left(\theta_{4}-\theta_{40}\right) \dot{\theta}_{4}+K s_{2}\left[\left(\theta_{4}-\theta_{3}\right)-\left(\theta_{4,0}-\theta_{3,0}\right)\right]\left(\dot{\theta}_{4}-\dot{\theta}_{3}\right)
$$

In order to calculate the necessary torque input by the driving DC motor, the torque generated by aerodynamic force is needed to transfer to the rotation center of the crank connecting 

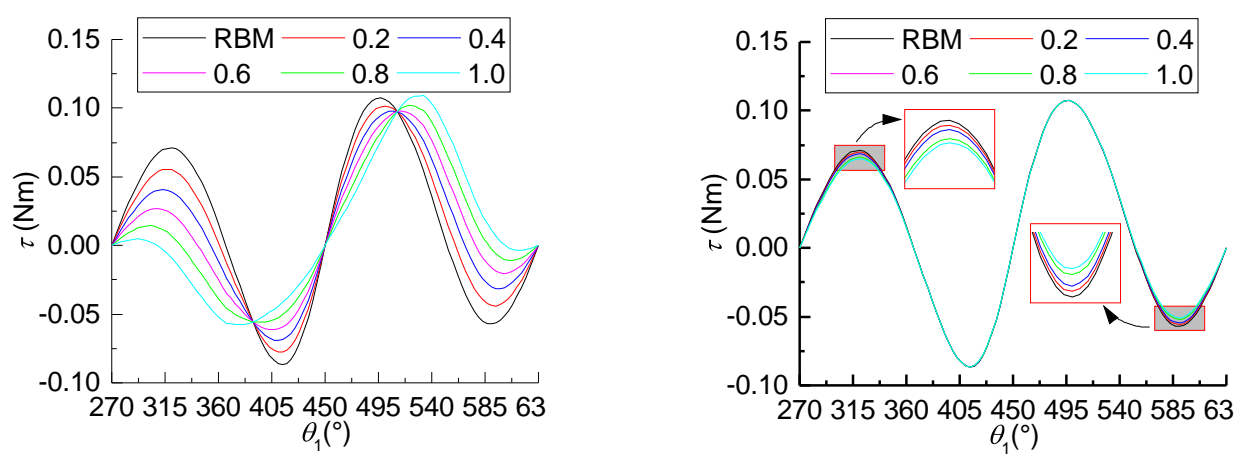

Figure 3.11: Variation of the input torque from the motor in function of the torsional stiffness of virtual springs at joint $\mathrm{C}$ and D. Left: $K_{1}=0(\mathrm{RBM}), 0.2,0.4,0.6,0.8,1 \mathrm{Nm} / \mathrm{rad}$ while $K_{2}=0$ $\mathrm{Nm} / \mathrm{rad}$; Right: $K_{2}=0$ (RBM), 0.2, $0.4,0.6,0.8,1 \mathrm{Nm} / \mathrm{rad}$ whereas $K_{1}=0 \mathrm{Nm} / \mathrm{rad}$. In both cases, $\theta_{3,0}$ and $\theta_{4,0}$ equal $0^{\circ}$ and $90^{\circ}$, respectively.

to the motor. The equivalent aerodynamic torques produced by flapping wing can be expressed in following equations according to (4),

$$
\left(\tau_{a}\right)_{e}=-\frac{\omega_{3}}{\omega_{1}} \tau_{a}=-\dot{\theta}_{3} \tau_{a}
$$

For maintaining the MAV flying stably, the drive motor has to overcome the torques generated by compliant joints and aerodynamic forces. Therefore, the total required input torque of the system from the motor is given as

$$
\tau_{m}=\tau_{s}+\left(\tau_{a}\right)_{e}
$$

Substituting Eqs. (3.24) and (3.25) into Eq. (3.26), therefore,

$$
\begin{aligned}
\tau_{m} & =\frac{1}{3} m_{8} r_{8}^{2} \alpha_{3} \dot{\theta}_{3}+m_{8} r_{4}^{2} \alpha_{4} \dot{\theta}_{4}-\tau_{a} \dot{\theta}_{3}+K s_{1}\left(\theta_{4}-\theta_{40}\right) \dot{\theta}_{4} \\
& +K s_{2}\left[\left(\theta_{4}-\theta_{3}\right)-\left(\theta_{4,0}-\theta_{3,0}\right)\right]\left(\dot{\theta}_{4}-\dot{\theta}_{3}\right)
\end{aligned}
$$

Comparing Eq. 3.13 with Eq. (3.27), it can be found that these two equations are the same, which demonstrate that rigid-body dynamics-based method could be as effective as the method based on the principle of virtual work. 

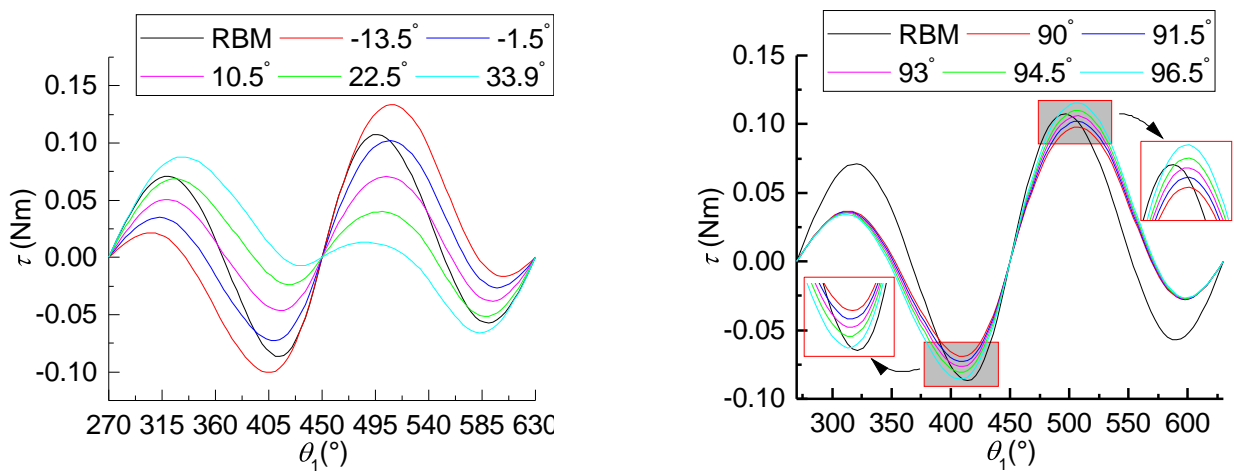

Figure 3.12: Variation of the input torque from the motor in function of the neutral angular positions at joint $\mathrm{C}$ and $\mathrm{D}$. :Left: $\mathrm{RBM}, \theta_{3,0}=-13.5^{\circ},-1.5^{\circ}, 10.5^{\circ}, 22.5^{\circ}, 33.9^{\circ}$ while $\theta_{4,0}=90^{\circ}$; Right: RBM, $\theta_{4,0}=90^{\circ}, 91.5^{\circ}, 93^{\circ}, 94.5^{\circ}, 96.5^{\circ}$ while $\theta_{3,0}=0^{\circ}$. In both cases, $K_{1}$ and $K_{2}$ equal 0.4 and $0.6 \mathrm{Nm} / \mathrm{rad}$, respectively.

\subsection{Optimization Design}

It can be found from the Eq. 3.13) or Eq. 3.27) that the primary attributes, including the torsional stiffness of the virtual spring at the compliant joint $K_{i}(i=1,2)$ and neutral angular position $\theta_{j, 0}(j=3,4)$, influence the input torque from the motor. The effect of each attribute on the input torque is shown in Fig. 3.11 and 3.12 respectively. As for the Fig. 3.11 (Left), the peak torques over a cycle fall down when the stiffness at compliant joint $\mathrm{C} K_{1}$ increases from 0 (pure rigid-body mechanism, RBM) to $0.6 \mathrm{Nm} / \mathrm{rad}$ and then the values rise from 0.6 to 1 $\mathrm{Nm} / \mathrm{rad}$. The value of the peak torque is the minimum when $K_{1}$ equals $0.6 \mathrm{Nm} / \mathrm{rad}$. The value of the trough torque always increases when $K_{1}$ varies from 0 to $0.8 \mathrm{Nm} / \mathrm{rad}$ and then slightly decreases when $K_{1}=1 \mathrm{Nm} / \mathrm{rad}$. From the Fig. 3.11 (Right), it is clear that the maximum and minimum values of the input torque seem to be the same with the variation of the stiffness at joint $\mathrm{D} K_{2}$ from 0 to $1 \mathrm{Nm} / \mathrm{rad}$ with a increment $0.2 \mathrm{Nm} / \mathrm{rad}$. Comparing with the pure rigid-body mechanism (RBM), the peak torques during the downstroke (from $270^{\circ}$ to $450^{\circ}$ ) slightly decrease while the values at the trough points during the upstroke (from $450^{\circ}$ to $630^{\circ}$ ) lightly increase with the rise of the stiffness at joint D.

In the Fig. 3.12 (Left), the peak torque in a cycle is the minimum for the compliant mechanism when the value of $\theta_{3,0}$ is chosen to be $10.5^{\circ}$. The minimum value is smaller than that of a RBM. While $\theta_{3,0}$ varies from $-1.5^{\circ}$ to $33.9^{\circ}$, none of the peak torque exceed the maximum torque needed for a RBM. From Fig. 3.12 (Right), it can be seen that the peak 
torque during a whole flapping period is the minimum when the neutral angle at joint $\mathrm{D}$ $\theta_{4,0}$ equals $\pi / 2$. With the rise of the angle $\theta_{4,0}$, the absolute values at the peak and trough points increase, which clearly violates the objective of minimizing the peak torque and thereby lowering the shock of the motor. When the values of $\theta_{4,0}$ are bigger than $93^{\circ}$, the maximum input torque required for a compliant mechanism are even greater than the corresponding value for a RBM, which suggests that choosing a proper angular position of $\theta_{4,0}$ is important in order to minimize the peak torque. Thus, for the purpose of reducing the torque required as possible, the optimization of $K_{1}, K_{2}, \theta_{3,0}$ and $\theta_{4,0}$ is the key. The objective function to optimization can be formulated as follows:

$$
F_{o b j}=\min \left(\tau_{m_{\max }}-\tau_{m_{\min }}\right)
$$

The corresponding constraint conditions are:

$$
K_{1} \in\left[K_{1 l}, K_{1 u}\right], K_{2} \in\left[K_{2 l}, K_{2 u}\right], \theta_{3,0} \in\left[\theta_{3 l}, \theta_{3 u}\right], \theta_{4,0} \in\left[\theta_{4 l}, \theta_{4 u}\right]
$$

where $\tau_{m_{\max }}$ and $\tau_{m_{\min }}$ are the maximum and minimum values of input torque over a flapping cycle, respectively. $K_{i l}, K_{i u}(i=1,2), \theta_{j, l}, \theta_{j, u}(j=3,4)$ are the lower and upper bounds of $K_{i}(i=1,2), \theta_{j, 0}(j=3,4)$, respectively. The situation with four constraints are referred to as Case $I$ in the following. According to the analysis on the four primary attributes in previous section, those bound parameters used for the optimization can be determined for the case at hand as shown in Table 3.4. In (13), the optimization problem is treated assuming that the two compliant joints have the same revolute stiffness and both their neutral angular positions are located in the middle of their motion ranges. Such assumptions can be expressed as,

$$
\begin{gathered}
K_{1} \in\left[K_{1 l}, K_{1 u}\right], K_{2} \in\left[K_{2 l}, K_{2 u}\right], K_{1}=K_{2} \\
\theta_{3,0}=\frac{1}{2}\left(\theta_{3_{\max }}+\theta_{3_{\min }}\right), \theta_{4,0}=\frac{1}{2}\left(\theta_{4_{\max }}+\theta_{4_{\min }}\right)
\end{gathered}
$$

where $\theta_{j_{\max }}, \theta_{j_{\min }}(j=3,4)$ are the upper and lower limits of the motion ranges with respect to $\theta_{j}$. Their values can be obtained in Fig. 3.9. This case is referred to as Case II in the following. 
Table 3.4: Parameters used for design optimisation

\begin{tabular}{ccc}
\hline \hline Parameter & Lower Bound & Upper Bound \\
\hline$K_{1}(\mathrm{Nm} / \mathrm{rad})$ & 0 & 0.8 \\
$K_{2}(\mathrm{Nm} / \mathrm{rad})$ & 0 & 1 \\
$\theta_{3,0}\left(^{\circ}\right)$ & -1.5 & 33.9 \\
$\theta_{4,0}\left({ }^{\circ}\right)$ & 90 & 93 \\
\hline \hline
\end{tabular}

\subsubsection{Result Analysis}

Similarly, objective function $(3.28)$ is a single-objective optimization problem with constraint conditions. The HMPSO algorithm mentioned above is employed for the optimisation. The results of the optimization process are in shown in Table 3.5. According to the optimization results, several optimum values of the joint torsional stiffness and neutral angular position appear to stand at the bounds. This may indicate that, the values of the torsional stiffness at compliant joints have a significant influence on the input torque and the greater the values of the stiffness are, the smaller the difference between the maximum and minimum input torque is. Furthermore, neutral positions at compliant joints tend to locate the centres of their respective angle variation ranges, which to some extend echo in concert with the assumptions on neutral positions in (13).

The input torques required for a rigid-body mechanism and compliant transmission mechanisms with different limitations (CTM Cases I and II) are shown in Fig. 3.13. As it can be noticed, both the mechanisms employing compliant joints with well-tuned parameters can reduce the peak to torque required from the motor comparing to the counterpart of the fully rigid-body mechanism (labelled RBM). Specifically, the maximum input torque for CTM I is reduced by $66.0 \%$ and the minimum torque is decreased by $71.7 \%$ compared to the RBM. Similarly, for CTM II, the input torque relative to the pure RMB, is reduced by $40.4 \%$ and $73.9 \%$, for maximum and minimum input torques, respectively. In addition, it can also be found that the peak torque optimized of the compliant mechanism obtained by using the limitations Case I is smaller that the corresponding gotten by utilizing the constraints Case II, which shows that the constraint method provided in this paper is better than that used in (13). In fact, the constraint conditions Case II is only a specific subset of the limitations Case I. 
Table 3.5: Optimisation results for two kinds of constraint conditions

\begin{tabular}{ccccc}
\hline \hline Condition & $K_{1}(\mathrm{Nm} / \mathrm{rad})$ & $K_{2}(\mathrm{Nm} / \mathrm{rad})$ & $\theta_{3,0}\left({ }^{\circ}\right)$ & $\theta_{4,0}\left({ }^{\circ}\right)$ \\
\hline Case I & 0.8 & 1 & 12.2 & 90 \\
Case II & 0.8 & 0.8 & 10.2 & 93.3 \\
\hline \hline
\end{tabular}

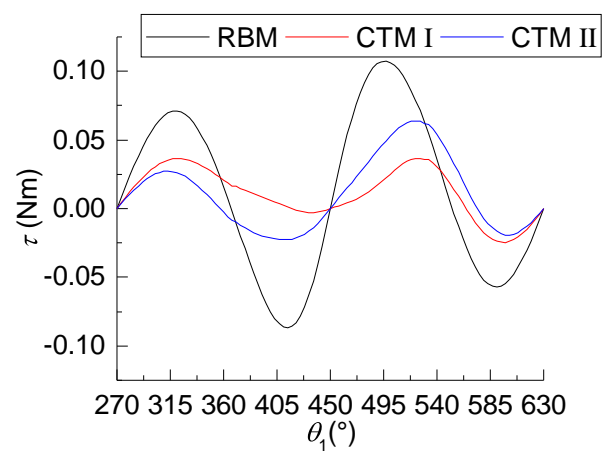

Figure 3.13: Input torque required of the flapping mechanism with three different transmission systems: Rigid-body Mechanism (RBM); Compliant Transmission Mechanism with constraints Case I (CTM I); Compliant Transmission Mechanism with constraints Case II (CTM II).

\subsection{Conclusions}

In this section, we have presented two compliant transmission mechanisms for a flapping-wing aerial vehicle. The compliant joints in both cases can be treated as revolute joints with virtual torsional springs. For the first-version, design, optimisation and Von Mises stress are presented. Later, a second improved compliant transmission mechanism is proposed. Virtual-work-based and rigid-body dynamics-based methodologies were respectively adopted to analyse the input torque needed by a rotational motor to produce the flapping movements. Following the analysis, the optimization design of compliant joints in terms of the stiffness of virtual spring and the initial angular position has been carried out. The results of the optimization shown that, theoretically, up to $66.0 \%$ of the maximum input torque, and up to $73.9 \%$ of the minimum torque could be reduced compared to the full rigid-body mechanism. The results obtained demonstrate that the compliant mechanism with proper design can significantly reduce the peak input torque and avoid the sharp shocks of the driving motor. Future work will be devoted to the physical instantiation of the mechanism using the optimized theoretical values for the key parameters obtained in this study. 


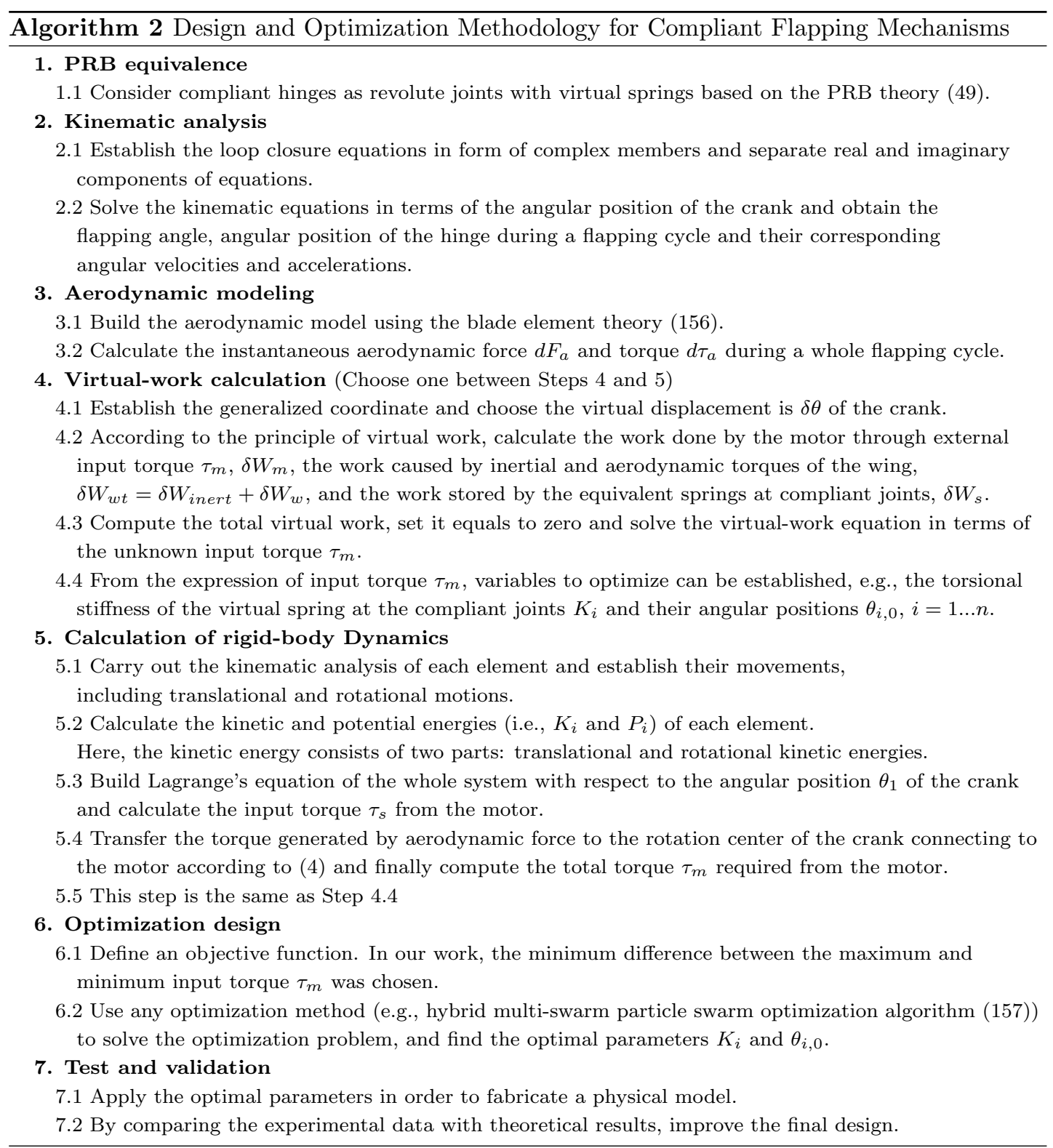

Compared to other optimization methods proposed (see, e.g. (4, 147)), our method are suitable for pseudo-rigid models since internal interaction forces do not need to be considered. Furthermore, the elastic potential energy stored in the compliant joints can be easily taken into account. The detail work process of the two methods written in pseudo code is shown in algorithm 2, It assumes a rotational actuator (DC motor) used for flapping, but it can be generalized to the design of other compliant transmission mechanisms. 


\section{Attitude Control and Simulation of a Flapping-wing Micro Air Vehicle}

\subsection{Introduction}

In this chapter, we will focus on the flight control of a flapping-wing micro air vehicle (FWMAV). Though many controller strategies (59) (60) (61) (62) (63) have been proposed for the flight of a FWMAV, as pointed out in Subsection 1.1.2, Chapter 1, a neural-network or learning-type control concept would have more advantages in adaptability and robustness than traditional control methodologies in complex flight environment (64) (65) (71). Meanwhile, considering the uncertainty in dynamic model of a FWMAV, a fuzzy neural network method would be a promising way to realize the stable control of the FWMAV.

Fuzzy neural network (FNN) is a hybrid intelligent system that combines the human-like reasoning style of fuzzy systems with the learning and connectionist structure of neural networks (159). The combination of the two techniques can not only avoid the lacking of interpretability for neural networks, but also eliminate poor learning capability of fuzzy systems. So far, the general type-1 FNNs have been successfully applied in a wide range of engineering issues, like temperature control of a reheating furnace (160), state-of-charge estimation of batteries (161), pattern classification (162), and traffic flow prediction (163). Though the knowledge of experts and experimental data based on available linguistic or numeric expressions can be integrated into the ordinary type-1 FNNs, however, in many cases the linguistic or numeric expressions are 
associated with various types of uncertainty that should be taken into consideration. Currently, employing type-2 fuzzy sets is an efficient approach to capture the factor of uncertainty. Type-2 fuzzy sets own fuzzy membership functions (MFs), which make them have a stronger capability to express the uncertainties, compared with type-1 counterparts whose MFs are crisp number. In this regard, type-2 FNNs (T2FNNs) appear to outperform type-1 FNNs in dealing with uncertain information. In addition, as mentioned in (159), FNNs can be regarded as parallel computational models with adaptive nature, which makes them possible to directly and effectively account for model's uncertainties caused by different sources and meanwhile contributes to the robustness and stability of the overall system. Therefore, in this study, we put forward a Takagi-Sugeno-Kang (TSK) T2FNN with adaptive learning capabilities to control attitude positions of a FWMAV. Meanwhile, the uncertainties of system and extra disturbances from the environment were also considered in this chapter.

The main structure of this chapter consists of four sections. First of all, a dynamic model of a FWMAV was briefly introduced on the basis of the work in (124). Secondly, a control frame based on a feedback-error-learning concept proposed in (164) was described. Within the structure, a T2FNN with triangular type-2 fuzzy membership functions was incorporated. To enhance the adaptability and robustness of the controller, sliding mode control (SMC) theory-based learning approach was taken into the T2FNN and the parameter update rules for the T2FNN were also derived. Meanwhile, it's also proved, for a second-order system under control, adding an auxiliary control term could guaranteed that the whole system is stable (165). Thirdly, the control method was applied into the dynamic control introduced and simulation results obtained were also shown in the section. The final part is the conclusion of this chapter.

\subsection{Dynamic Model of FWMAV}

As is known to all, it is very difficult to build the dynamic model of a FWMAV due to the unsteady, low Reynolds number flows experienced by the vehicle. Many of the dynamics models presented in the literature focus on the standard aircraft model. Deng et al. (55) used a standard six degrees of freedom (DoFs) nonlinear aircraft model and incorporated aerodynamics and actuator models to model the flight of a micromechanical flying insect. Khan (166) presented the modelling and simulation of flapping wing micro air vehicles based on the standard aircraft model. Simulations are presented for a hover condition by utilizing a quasi-steady aerodynamic model and the coefficient for the aerodynamic model are determined from a robotic flapper. 
Duan et al. (124) developed a flight dynamic model for an ornithopter for the purpose of attitude control. In addition, multi-body dynamic methods are also appeared in the literature for modelling flapping wing flight. Lasek et al. (167) developed a multi-body model of flapping wing flight of an ornithopter. In their model, wings have two degrees of freedom (DoFs): flapping and lagging. In addition, Grauer et al. (57) derived multi-body motion equations of an ornithopter with five rigid bodies (i.e., a central body, two wings, and two linkages for tail). Only one DoF is allowed for each rigid body. Furthermore, the transformations of those equations used for robotic control was derived for integrating nonlinear control techniques available. Recently, the model was further expanded for testing and system identification of the ornithopter (168).

In this thesis, in order to conduct control research conveniently, the dynamic model proposed by Duan et al. (124)(125) is employed for attitude control. The detailed information on the model is given as follows:

$$
\left\{\begin{array}{l}
\ddot{x}=f(x, \dot{x})+\tilde{f}+g(x) u+d \\
y=x
\end{array}\right.
$$

where $x=[\theta, \psi, \gamma]^{T}, \theta, \psi, \gamma$ are the Euler angles, i.e. pitch, yaw, roll angles, respectively; $f=\left[f_{1}, f_{2}, f_{3}\right]^{T} ; u \in R^{3}$ is a control signal; $\tilde{f}, d \in R^{3}$ are the uncertainties of the system and disturbances from extra environment, respectively. $g(x)$ is a control coefficient matrix. The details of $f_{1}, f_{2}, f_{3}$ and $g(x)$ are expressed as follows,

$$
\begin{gathered}
f_{1}=[p-q \tan \theta \cos \gamma+r \tan \theta \sin \gamma][q \cos \gamma-r \sin \gamma] \\
+\frac{\sin \gamma}{J_{y}}\left[\left(J_{z}-J_{x}\right) p r+M_{b y}\right]+\frac{\cos \gamma}{J_{z}}\left[\left(J_{x}-J_{y}\right) p q+M_{b z}\right] \\
f_{2}=\frac{\tan \theta}{\cos \theta}[q \sin \gamma+r \cos \gamma][q \cos \gamma-r \sin \gamma] \\
-\frac{1}{\cos \theta}[p-q \tan \theta \cos \gamma+r \tan \theta \sin \gamma][q \sin \gamma+r \cos \gamma] \\
+\frac{\cos \gamma}{J_{y} \cos \theta}\left[\left(J_{z}-J_{x}\right) p r+M_{b y}\right]-\frac{\sin \gamma}{J_{z} \cos \theta}\left[\left(J_{x}-J_{y}\right) p q+M_{b z}\right] \\
f_{3}=\frac{1}{\cos { }^{2}(\theta)}[q \sin (\gamma)+r \cos (\gamma)][r \sin (\gamma)-q \cos (\gamma)] \\
+\tan (\theta)[p-q \tan (\theta) \cos (\gamma)+r \tan (\theta) \sin (\gamma)][q \sin (\gamma)+r \cos (\gamma)] \\
+\frac{1}{J_{x}}\left[\left(J_{y}-J_{z}\right) q r+M_{b x}\right]-\frac{\tan (\theta) \cos (\gamma)}{J_{y}}\left[\left(J_{z}-J_{x}\right) p r+M_{b y}\right] \\
+\frac{\tan \theta \sin \gamma}{J_{z}}\left[\left(J_{x}-J_{y}\right) p q+M_{b z}\right]
\end{gathered}
$$




$$
g(x)=\left[\begin{array}{ccc}
\cos (\gamma+\tilde{\gamma}) & \sin (\gamma+\tilde{\gamma}) & 0 \\
\sin (\gamma+\tilde{\gamma}) / \cos (\theta+\tilde{\theta}) & \cos (\gamma+\tilde{\gamma}) / \cos (\theta+\tilde{\theta}) & 0 \\
\tan (\theta+\tilde{\theta}) \sin (\gamma+\tilde{\gamma}) & -\tan (\theta+\tilde{\theta}) \cos (\gamma+\tilde{\gamma}) & 1
\end{array}\right]
$$

In Eqs. 4.2) - 4.4 $, J_{x}, J_{y}, J_{z}$ are inertial moments in $x, y, z$ directions in the body frame. $M_{b x}, J_{b y}, J_{b z}$ are the aerodynamic moments of body frame. $\tilde{\theta}, \tilde{\gamma}$ are perturbation angles of attitude in Eq. 4.5. . p , $q, r$ represent rotating velocities in $x, y, z$ directions in body coordinate frame, respectively. They can be written as

$$
\left[\begin{array}{l}
p \\
q \\
r
\end{array}\right]=\left[\begin{array}{ccc}
0 & \sin \theta & 1 \\
\sin \gamma & \cos \theta \cos \gamma & 0 \\
\cos \gamma & -\cos \theta \sin \gamma & 0
\end{array}\right]\left[\begin{array}{c}
\dot{\theta} \\
\dot{\psi} \\
\dot{\gamma}
\end{array}\right]
$$

\subsection{Feedback-error-learning Control Frame}

Feedback-error-learning (FEL) control frame proposed in (164) was initially applied to control of robotic manipulators. This frame normally consists of two parallel feedback controllers. One is a traditional feedback controller and the other is an intelligent controller. As shown in Fig. 4.1, a T2FNN is integrated into the FEL control frame, which works in parallel with a proportional-derivative $(\mathrm{PD})$ controller. Here, the neural network is an intelligent controller while the PD controller is a conventional controller. According to the indication in Fig. 4.1, the output of the PD controller, $u_{p d}$, is used to update the parameters of the FNN. The conventional PD controller acts as an ordinary feedback controller to ensure the stability of the system. The $\mathrm{PD}$ control law is described as follows

$$
u_{p d}=K_{p} e+K_{d} \dot{e}
$$

where $e=x_{r}-x$ is the feedback error, $\dot{e}=\dot{x}_{r}-\dot{x}$ is the time derivative of the feedback error. $K_{p}$ and $K_{d}$ are the controller gains. The auxiliary term, $u_{a}$, is a robust term used for enhancing the robustness of the whole system.

\subsection{Type-2 Fuzzy Neural Network with Triangular Type- 2 MFs}

In this section, an interval A2-C0 fuzzy neural network is considered to be embedded in the above FEL frame, in which type-2 triangular MFs (T2TMFs) are used in the premise part and crisp numbers are chosen in the consequent part. 


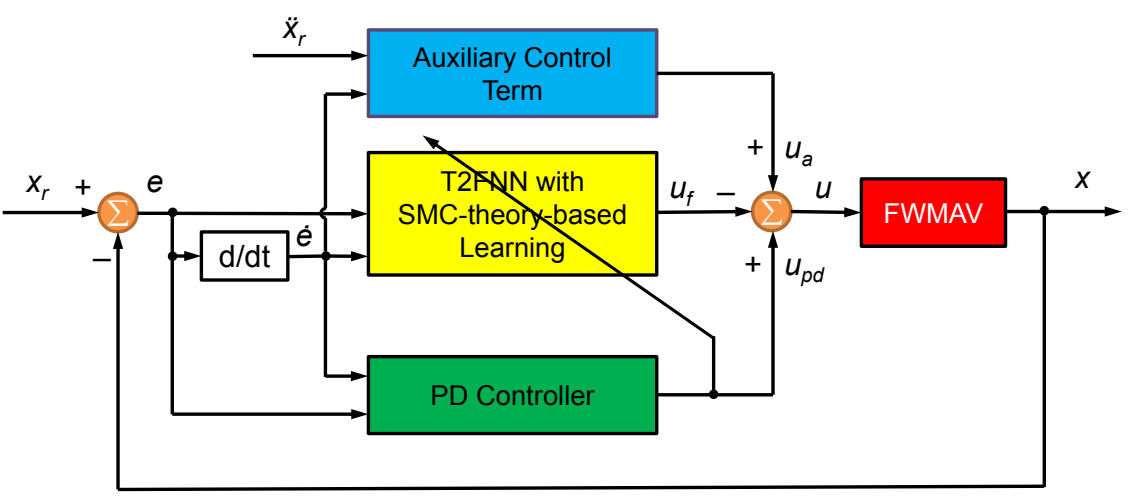

Figure 4.1: Feedback-error-learning control frame incorporating a T2FNN structure.

\subsubsection{Type-2 Triangular Membership Functions}

The type-2 membership functions employed in this chapter is triangular. The T2TMF is an extension of type- 1 triangular MF (T1TMF) whose mathematical expression is described as

$$
\mu(x)= \begin{cases}1-\left|\frac{x-c}{d}\right|, & |x-c|<d \\ 0, & \text { Others }\end{cases}
$$

where $c$ and $d$ are the center and the width of the membership function, respectively. $x$ is an input. In Fig. 4.2 (a), triangular type-2 fuzzy membership functions with uncertain width is shown. As shown in this figure, the T2TMF center $c$ is fixed while the T2TMF width $d$ is variable. $\underline{d}$ and $\bar{d}$ are the lower and upper bounds of $d$, respectively. In contrast, Fig. 4.2 (b) is the case of T2TMF with uncertain center, in which the center $c$ of T2TMF is changeable while the width $d$ is firmed. $\underline{c}$ and $\bar{c}$ are denoted as the lower and upper limits of $c$, respectively. In both cases, $\underline{\mu}$ and $\bar{\mu}$ represent the corresponding lower and upper T2TMFs, respectively. In this study, both of these two different T2TMFs are considered to carry out the attitude control of a FWMAV.

As indicated in Fig. 4.1, the T2FNN has two inputs, i.e., $e$ and $\dot{e}$. Thereby, the T2TMFs for $e$ and $\dot{e}$ could be expressed as $\underline{\mu}_{1 i}(e), \bar{\mu}_{1 i}(e), \underline{\mu}_{2 j}(\dot{e})$ and $\bar{\mu}_{2 j}(\dot{e})$. For those T2TMFs with 


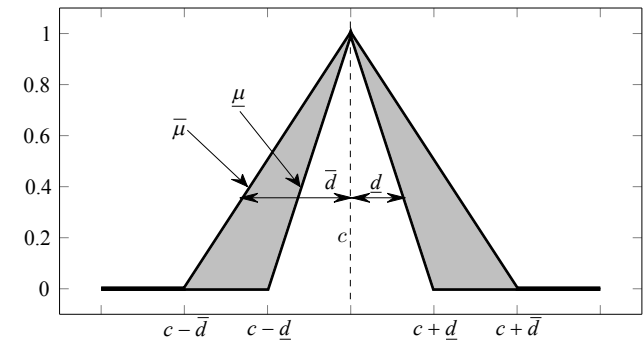

(a)

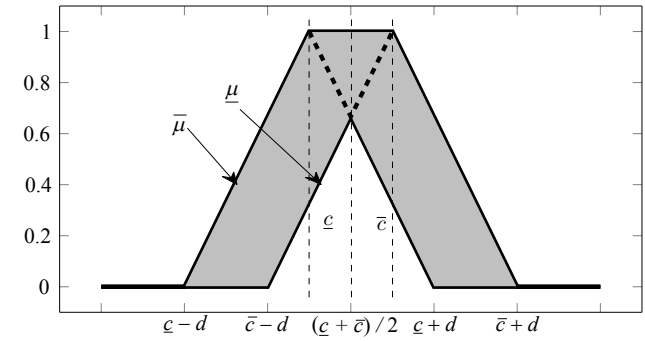

(b)

Figure 4.2: Triangular type-2 fuzzy set with uncertain width (a) and uncertain center (b).

uncertain width, they can be written as

$$
\begin{aligned}
& \underline{\mu}_{1 i}(e)= \begin{cases}1-\left|\frac{e-c_{1 i}}{\underline{d}_{1 i}}\right|, & \left|e-c_{1 i}\right|<\underline{d}_{1 i} \\
0, & \text { Others }\end{cases} \\
& \bar{\mu}_{1 i}(e)= \begin{cases}1-\left|\frac{e-c_{1 i}}{\bar{d}_{1 i}}\right|, & \left|e-c_{1 i}\right|<\bar{d}_{1 i} \\
0, & \text { Others }\end{cases} \\
& \underline{\mu}_{2 j}(\dot{e})= \begin{cases}1-\left|\frac{\dot{e}-c_{2 j}}{\underline{d}_{2 j}}\right|, & \left|\dot{e}-c_{2 j}\right|<\underline{d}_{2 j} \\
0, & \text { Others }\end{cases} \\
& \bar{\mu}_{2 j}(\dot{e})= \begin{cases}1-\left|\frac{\dot{e}-c_{2 j}}{\bar{d}_{2 j}}\right|, & \left|\dot{e}-c_{2 j}\right|<\bar{d}_{2 j} \\
0, & \text { Others }\end{cases}
\end{aligned}
$$

Similarly, the T2MFs with uncertain center for $e$ and $\dot{e}$ are

$$
\begin{gathered}
\underline{\mu}_{1 i}(e)= \begin{cases}1-\left|\frac{e-\bar{c}_{1 i}}{d_{1 i}}\right|, & \bar{c}_{1 i}-d_{1 i}<e<\frac{\underline{c}_{1 i}+\bar{c}_{1 i}}{2} \\
1-\left|\frac{e-\underline{c}_{1 i}}{d_{1 i}}\right|, & \frac{\underline{c}_{1 i}+\bar{c}_{1 i}}{2} \leq e<\underline{c}_{1 i}+d_{1 i} \\
0, & \text { Others }\end{cases} \\
\bar{\mu}_{1 i}(e)= \begin{cases}1-\left|\frac{e-\underline{c}_{1 i}}{d_{1 i}}\right|, & \underline{c}_{1 i}-d_{1 i}<e<\underline{c}_{1 i} \\
1, & \underline{c}_{1 i} \leq e \leq \bar{c}_{1 i} \\
1-\left|\frac{e-\bar{c}_{1 i}}{d_{1 i}}\right|, & \bar{c}_{1 i}<e<\bar{c}_{1 i}+d_{1 i} \\
0, & \text { Others }\end{cases}
\end{gathered}
$$




$$
\begin{gathered}
\underline{\mu}_{2 j}(\dot{e})= \begin{cases}1-\left|\frac{\dot{e}-\bar{c}_{2 j}}{d_{2 j}}\right|, & \bar{c}_{2 j}-d_{2 j}<\dot{e}<\frac{\underline{c}_{2 j}+\bar{c}_{2 j}}{2} \\
1-\left|\frac{\dot{e}-\underline{c}_{2 j}}{d_{2 j}}\right|, & \frac{\underline{c}_{2 j}+\bar{c}_{2 j}}{2} \leq \dot{e}<\underline{c}_{2 j}+d_{2 j} \\
0, & \text { Others }\end{cases} \\
\bar{\mu}_{2 j}(\dot{e})= \begin{cases}1-\left|\frac{\dot{e}-\underline{c}_{2 j}}{d_{2 j}}\right|, & \underline{c}_{2 j}-d_{2 j}<\dot{e}<\underline{c}_{2 j} \\
1, & \underline{c}_{2 j} \leq \dot{e} \leq \bar{c}_{2 j} \\
1-\left|\frac{\dot{e}-\bar{c}_{2 j}}{d_{2 j}}\right|, & \bar{c}_{2 j}<\dot{e}<\bar{c}_{2 j}+d_{2 j} \\
0, & \text { Others }\end{cases}
\end{gathered}
$$

where $\left(\bar{c}_{1 i}-\underline{c}_{1 i}\right) / 2 \leq d_{1 i},\left(\bar{c}_{2 j}-\underline{c}_{2 j}\right) / 2 \leq d_{2 j}$.

\subsubsection{Interval Type-2 A2-C0 Takagi-Sugeno-Kang Model}

The Takagi-Sugeno-Kang (TSK) model was firstly proposed in (169) (170) for the type-1 fuzzy systems. After that, Mendel (171) further extended the type-1 fuzzy TSK model to type-2 fuzzy systems. Interval type-2 (IT2) TSK systems are TSK models where interval fuzzy sets are used to describe the level of uncertainty in the antecedents and/or consequents. IT2 TSK models have been categorised into three different classes (171): (a) both antecedent and consequent membership functions are IT2 fuzzy sets (A2-C2); (b) antecedent and consequent membership functions are IT2 and T1 fuzzy sets, respectively (A2-C1); (c) antecedent membership functions are type-2 and consequent are crisp numbers (A2-C0). A2-C0 fuzzy model is the simplest among the three. The use of crisp numbers as consequent lessens the computational burden of type- 2 fuzzy systems considerably. On the base of the Mendel's work, Begian et al. (172) further analysed the stability of IT2 A2-C0 TSK fussy systems to guarantee the methods stable while employed in control applications. Here, we consider the simplest IT2 A2-C0 TSK model whose consequent part is a $0^{\text {th }}$-order crisp number. The fuzzy inference rule of such model, $R_{i j}$, can be defined as follows:

$$
R_{i j}: \text { if } e \text { is } \tilde{A}_{1 i} \text { and } \dot{e} \text { is } \tilde{B}_{2 j} \text {, then } f_{i j}=\tilde{C}_{i j} .
$$

where $e$ and $\dot{e}$ are the inputs of the type-2 TSK model as shown in Layer 1, in Fig. $4.3 . \tilde{A}_{1 i}$ is the $i$ th type-2 fuzzy MF $(i=1, \ldots, I)$ of $e$ and $\tilde{B}_{2 j}$ is the $j$ th type-2 fuzzy MF $(j=1, \ldots, J)$ of $\dot{e}$, as displayed in Layer 2. $\tilde{C}_{i j}$ is the $i j$ th crisp number in consequents. $f_{i j}$ is the output 
of the rule $R_{i j}$ in Layer 3 . The firing strength of the rule using the prod T-norm operator is calculated as follows:

$$
\begin{aligned}
& \underline{W}_{i j}=\underline{\mu}_{1 i}(e) \underline{\mu}_{2 j}(\dot{e}) \\
& \bar{W}_{i j}=\bar{\mu}_{1 i}(e) \bar{\mu}_{2 j}(\dot{e})
\end{aligned}
$$

In Layer 4 as shown in Fig. 4.3, there are two summation blocks that respectively compute the sum of lower and upper output signals from the fuzzy inference rules and their corresponding firing strengths, respectively. Then, the results of Layer 4 feed to the Layer 5 to obtain the final output of the network. The output of the network is calculated as follows:

$$
u_{f}=q \sum_{i=1}^{I} \sum_{j=1}^{J} f_{i j} \widetilde{\widetilde{W}}_{i j}+(1-q) \sum_{i=1}^{I} \sum_{j=1}^{J} f_{i j} \widetilde{\bar{W}}_{i j}
$$

where $\widetilde{W}_{i j}$ and $\widetilde{W}_{i j}$ are the normalized values of the lower and upper firing strengths of the neuron $i j$ from the Layer 3 of the network:

$$
\begin{aligned}
\widetilde{W}_{i j} & =\frac{\underline{W}_{i j}}{\sum_{i=1}^{I} \sum_{j=1}^{J} \underline{W}_{i j}} \\
\widetilde{\bar{W}}_{i j} & =\frac{\bar{W}_{i j}}{\sum_{i=1}^{I} \sum_{j=1}^{J} \bar{W}_{i j}}
\end{aligned}
$$

Here, $0<\widetilde{W}_{i j}<1$ and $0<\widetilde{W}_{i j}<1$. According to the definitions in Eqs. 4.21 and 4.22 , $\sum_{i=1}^{I} \sum_{j=1}^{J} \widetilde{W}_{i j}=1$ and $\sum_{i=1}^{I} \sum_{j=1}^{J} \widetilde{W}_{i j}=1$.

\subsection{SMC Theory-based Learning Algorithm}

At present, two kinds of learning algorithms are widely used for T2FNNs. One is a gradient descent (GD)-based algorithm which requires the computation of partial derivatives or sensitivity functions to tune the parameters of T2FNNs. As pointed out in (173) (174), the shortcomings for this method are definite, like long computation time, slow learning and converging speed, difficult in stability analysis and easily trapped in local minimum. The other learning method is based on evolutionary theory, like genetic algorithm (175) (176), particle swarm optimization (160) (177) and ant colony optimization (178). In general, this evolutionary theory-based algorithm usually adopts a random basis to do a stochastic search, which brings with intensive 

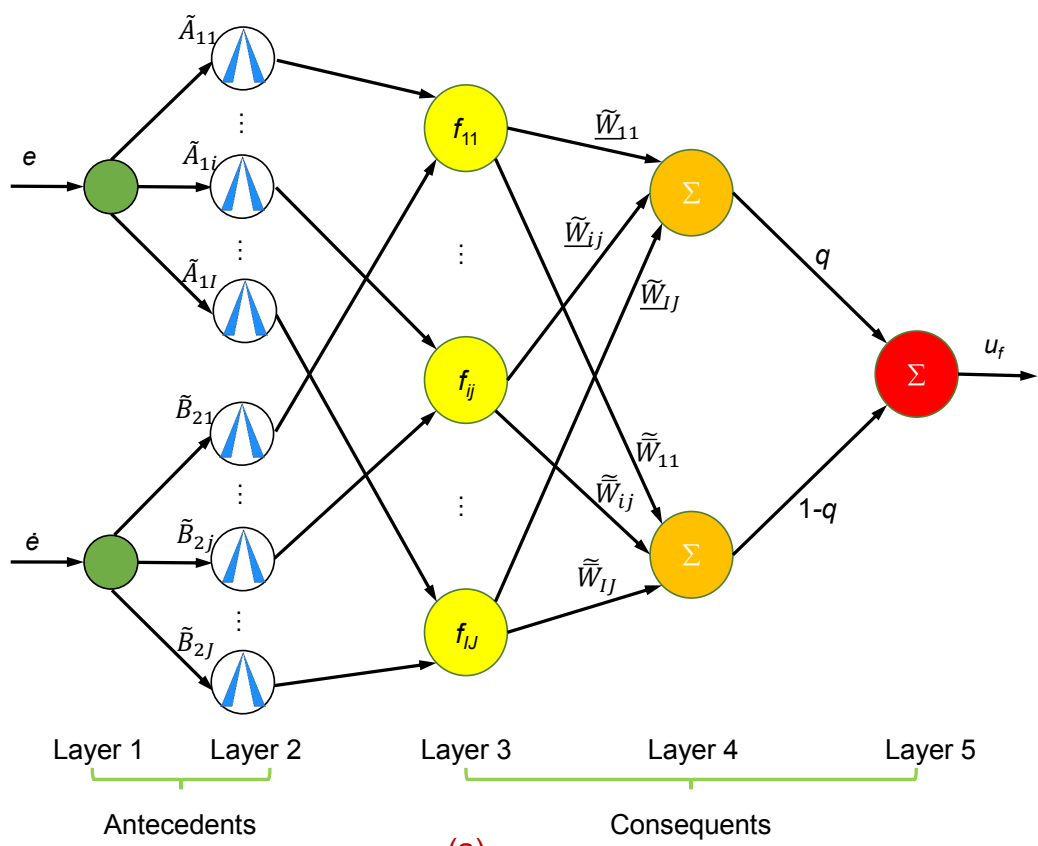

(a)
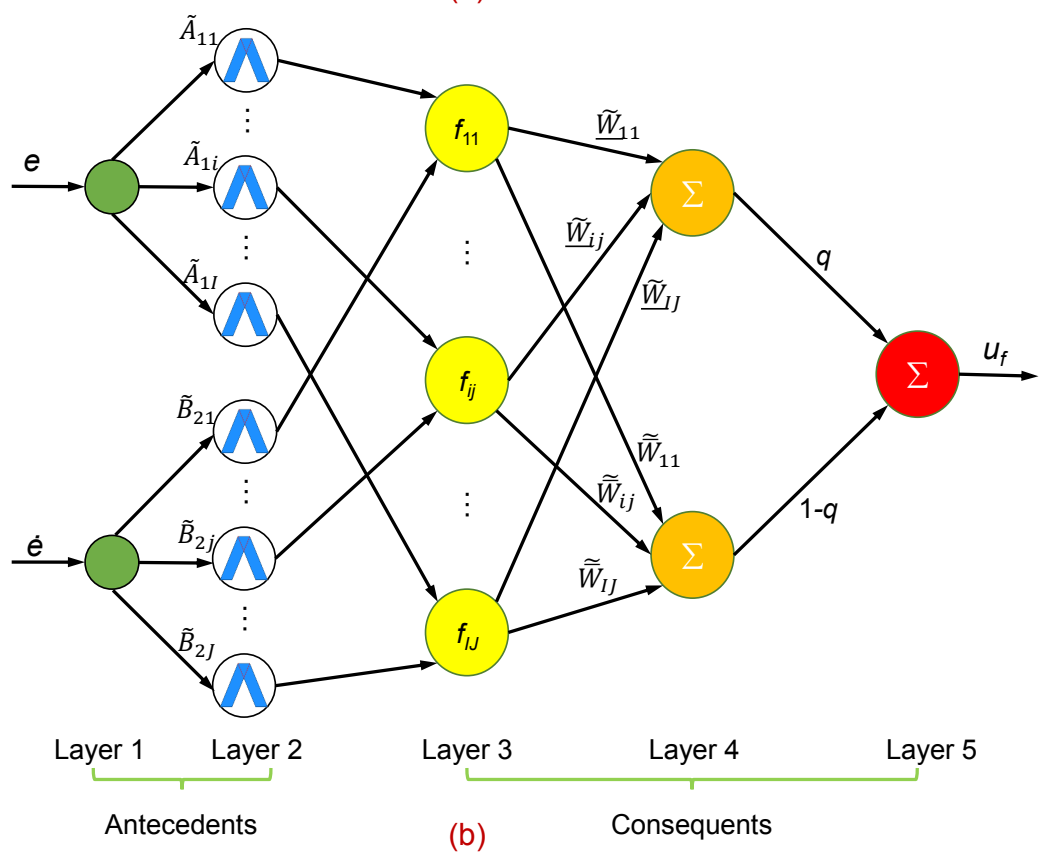

Figure 4.3: The structure of Interval type-2 A2-C0 TSK FNN using triangular MFs with uncertain width (a) and uncertain center (b).

computations and thereby causes a slower learning speed compared to the gradient-descent algorithm. Furthermore, the stability of such approach is questionable and the optimal values 
for the stochastic operators are difficult to derive (174).

To overcome these issues, SMC theory-based algorithms are proposed for the parameter update rules of type-1 FNNs (T1FNNs) as robust learning algorithms (179). SMC theorybased learning algorithms not only can make the overall system more robust but also can ensure faster convergence than the traditional learning techniques in online tuning of FNNs (180) (181). Moreover, the parameter update rules are much simpler when compared with gradient descentbased and evolutionary theory-based algorithms. Motivated by the successful results of these learning algorithms on T1FNNs, the derivation of SMC theory-based learning algorithms for the training of type-2 FNNs (T2FNNs) are also proposed (182). Therefore, in this paper, an SMC theory-based learning algorithm has been employed to tune both the premise and consequent parts of T2FNNs. Subsequently, the stability of the introduced learning algorithm is proved using Lyapunov approach.

\subsubsection{Parameter Update Rules for T2FNN}

Before we start, three vectors are defined in advance to prepare for the use in the following update rules. $\widetilde{\widetilde{W}}=\left[\widetilde{W}_{11}, \ldots, \underline{\widetilde{W}}_{i j}, \ldots, \widetilde{W}_{I J}\right]^{T}$ is a vector of the normalized lower firing strength signals. $\widetilde{W}(t)=\left[\widetilde{\bar{W}}_{11}, \ldots, \widetilde{W}_{i j}, \ldots, \widetilde{W}_{I J}\right]^{T}$ is a vector of the normalized upper firing strength signals. $\underline{\widetilde{F}}=\left[f_{11}, \ldots, f_{i j}, \ldots, f_{I J}\right]$ is an output vector of $I \times J$ inference rules. Moreover, we assume that the input signals $e(t)$ and $\dot{e}(t)$, and the time derivative of $\dot{e}(t)$ are bounded:

$$
|e(t)| \leq B_{e},|\dot{e}(t)| \leq B_{\dot{e}},|\ddot{e}(t)| \leq B_{\ddot{e}}, \forall t
$$

where $B_{e}, B_{\dot{e}}$ and $B_{\ddot{e}}$ are assumed to be some known positive constants. Meanwhile, $u$ and $\dot{u}$ are bounded signals, too, i.e. $|u(t)| \leq B_{u},|\dot{u}(t)| \leq B_{\dot{u}}, \forall t$, where $B_{u}$ and $B_{\dot{u}}$ are two unknown positive constants. In addition, the adaptive parameters, $q$ and $f_{i j}$ are bounded in the case of T2TMFs with uncertain center as follows: $\|q\| \leq B_{q},\left\|f_{i j}\right\| \leq B_{f}$, where $B_{q}$ and $B_{f}$ are some unknown positive constants.

As mentioned before, two kinds of interval type-2 triangular MFs are considered to integrated into the T2FNN. The parameter update rules for both cases are given in the following theorems.

Theorem 1

Given a T2FNN using T2TMFs with uncertain width and considering an arbitrary initial condition $u_{p d}(0)$ as well, $u_{p d}(t)$ will asymptotically converges to zero in a finite time if the 
update rules for adapting the parameters of the aforementioned T2FNN are chosen as

$$
\begin{aligned}
& \dot{c}_{1 i}=\dot{e}+\left(e-c_{1 i}\right) \eta \operatorname{sgn}\left(u_{p d}\right) \\
& \underline{\dot{d}}_{1 i}=-\frac{\underline{d}_{1 i}^{2}}{e-c_{1 i}} \eta \operatorname{sgn}\left(u_{p d}\right) \operatorname{sgn}\left(\frac{e-c_{1 i}}{\underline{d}_{1 i}}\right) \\
& \dot{\bar{d}}_{1 i}=-\frac{\bar{d}_{1 i}^{2}}{e-c_{1 i}} \eta \operatorname{sgn}\left(u_{p d}\right) \operatorname{sgn}\left(\frac{e-c_{1 i}}{\bar{d}_{1 i}}\right) \\
& \dot{c}_{2 j}=\ddot{e}+\left(\dot{e}-c_{2 j}\right) \eta \operatorname{sgn}\left(u_{p d}\right) \\
& \underline{\dot{d}}_{2 j}=-\frac{\underline{d}_{2 j}^{2}}{\dot{e}-c_{2 j}} \eta \operatorname{sgn}\left(u_{p d}\right) \operatorname{sgn}\left(\frac{\dot{e}-c_{2 j}}{\underline{d}_{2 j}}\right) \\
& \dot{\bar{d}}_{2 j}=-\frac{\bar{d}_{2 j}^{2}}{\dot{e}-c_{2 j}} \eta \operatorname{sgn}\left(u_{p d}\right) \operatorname{sgn}\left(\frac{\dot{e}-c_{2 j}}{\bar{d}_{2 j}}\right) \\
& \dot{f}_{i j}=\frac{-\left[q \widetilde{W}_{i j}+(1-q) \widetilde{\bar{W}}_{i j}\right] \beta \operatorname{sgn}\left(u_{p d}\right)}{[q \underline{\widetilde{W}}+(1-q) \widetilde{\widetilde{W}}]^{T}[q \underline{\widetilde{W}}+(1-q) \widetilde{\widetilde{W}}]} \\
& \dot{q}=\frac{-\beta \operatorname{sgn}\left(u_{p d}\right)}{F(\widetilde{\widetilde{W}}-\widetilde{\widetilde{W}})} \\
& \dot{\beta}=\frac{1}{2} \alpha\left|u_{p d}\right|
\end{aligned}
$$

Proof The reader is referred to Appendix 8.1 .

\section{Theorem 2}

Given a T2FNN using T2TMFs with uncertain center and considering an arbitrary initial condition $u_{p d}(0)$ as well, $u_{p d}(t)$ will asymptotically converges to zero in a finite time if the update rules for adapting the parameters of the aforementioned T2FNN are chosen as

$$
\begin{aligned}
& \dot{c}_{1 i}=\dot{e}-\eta d_{1 i} \operatorname{sgn}\left(u_{p d}\right) \operatorname{sgn}\left(\frac{e-\underline{c}_{1 i}}{d_{1 i}}\right) \\
& \dot{\bar{c}}_{1 i}=\dot{e}-\eta d_{1 i} \operatorname{sgn}\left(u_{p d}\right) \operatorname{sgn}\left(\frac{e-\bar{c}_{1 i}}{d_{1 i}}\right)
\end{aligned}
$$




$$
\begin{aligned}
& \dot{d}_{1 i}=\eta d_{1 i} \operatorname{sgn}\left(u_{p d}\right) \\
& \underline{\underline{c}}_{2 j}=\ddot{e}-\eta d_{2 j} \operatorname{sgn}\left(u_{p d}\right) \operatorname{sgn}\left(\frac{\dot{e}-\underline{c}_{2 j}}{d_{2 j}}\right) \\
& \dot{\bar{c}}_{2 j}=\ddot{e}-\eta d_{2 j} \operatorname{sgn}\left(u_{p d}\right) \operatorname{sgn}\left(\frac{\dot{e}-\bar{c}_{2 j}}{d_{2 j}}\right) \\
& \dot{d}_{2 j}=\eta d_{2 j} \operatorname{sgn}\left(u_{p d}\right) \\
& \dot{f}_{i j}=\frac{-\left[q \widetilde{W}_{i j}+(1-q) \widetilde{\bar{W}}_{i j}\right] \beta \operatorname{sgn}\left(u_{p d}\right)}{[q \underline{\widetilde{W}}+(1-q) \widetilde{\widetilde{W}}]^{T}[q \underline{\widetilde{W}}+(1-q) \widetilde{\widetilde{W}}]} \\
& \dot{q}=\frac{-\beta \operatorname{sgn}\left(u_{p d}\right)}{F(\widetilde{\widetilde{W}}-\widetilde{\bar{W}})} \\
& \dot{\beta}=\frac{1}{2} \alpha\left|u_{p d}\right|
\end{aligned}
$$

Proof The reader is referred to Appendix 8.2

\subsubsection{Stability Analysis}

According to the SMC theory (183), the sliding surface for the nonlinear system under control $S(e, \dot{e})$ is defined as

$$
S(e, \dot{e})=e+\xi \dot{e}
$$

with $\xi$ being a positive constant determining the slope of the sliding surface. If $\xi$ is taken as $\xi=K_{d} / K_{p}$, then

$$
S=\frac{1}{K_{p}}\left(K_{p} e+K_{d} \dot{e}\right)=\frac{1}{K_{p}} u_{p d}
$$

It has proved that $u_{p d}$ converges asymptotically to zero in a finite time in Appendix both 8.1 and 8.2 when the parameters of the T2FNN are adjusted as the rules from Eq. (4.24) to Eq. 4.32 or from Eq. 4.33 to Eq. 4.41. Therefore, $S$ converges asymptotically to zero in a finite time as well based on the relationship equation 4.43 and then a sliding motion will appear on the sliding manifold $S(e, \dot{e})=0$. This result demonstrates that, selecting $u_{p d}$ as a 
learning error for the adaptation of the T2FNN ensures the stability of the learning methods and makes the system under control.

Theorem 3

Besides that, as mentioned in (165), for a second order nonlinear dynamic structure (See Fig. 4.1 which consists of a PD controller working in parallel with a T2FNN, adding an auxiliary term, $u_{a}$, can enhance the stability of the control loop and lower the requirements of stability conditions, especially that PD controller is no need to enable the system stable. In this thesis, we set $u_{a}=K_{a} \operatorname{sgn}(\dot{e})+\ddot{x}_{r}$, where $\dot{K}_{a}=\lambda|\dot{e}|, \lambda>0$. The detail of proof can be found in Appendix 8.3

\subsection{Simulation Results and Analysis}

In this section, the proposed control method is to be applied to the dynamic model of a flappingwing MAV for realizing its attitude control. The sample time is selected as 0.001s while simulating. Other initial parameters for simulation are listed as follows: $\theta=\psi=\gamma=0^{\circ}$. The perturbation of attitude angles are $\tilde{\theta}=0.3^{\circ} \sin (\pi t / 4), \tilde{\psi}=0.5^{\circ} \sin (\pi t / 8), \tilde{\gamma}=0.2^{\circ} \sin (\pi t / 3)$, respectively. The uncertain term from the system is $\tilde{f}=\left[1.5^{\circ} \cos t, 1.2^{\circ} \cos t, 0.8^{\circ} \cos t,\right]^{T}$ while the disturbance from extra environment is $d=\left[2.0^{\circ} \sin t, 1.6^{\circ} \sin t, 1.0^{\circ} \sin t,\right]^{T}$. The desired attitude angles are considered in two cases. In the first, the sinusoidal attitude angles are selected as references, which are given as

$$
x_{r}= \begin{cases}\theta_{r}=15^{\circ} \sin \left(\frac{\pi t}{4}+\frac{\pi}{8}\right), & 0 \leq t \leq 24 \mathrm{~s} \\ \psi_{r}=45^{\circ} \sin \left(\frac{\pi t}{8}-\frac{\pi}{6}\right), & 0 \leq t \leq 24 \mathrm{~s} \\ \gamma_{r}=10^{\circ} \sin \left(\frac{\pi t}{3}+\frac{\pi}{12}\right), & 0 \leq t \leq 24 \mathrm{~s}\end{cases}
$$

For the second case, the tracking attitude signals with step variations are chosen as

$$
x_{r}= \begin{cases}\theta_{r}=-15^{\circ}, \psi_{r}=-45^{\circ}, \gamma_{r}=10^{\circ}, & 0 \leq t<5 \mathrm{~s} \\ \theta_{r}=0^{\circ}, \psi_{r}=0^{\circ}, \gamma_{r}=0^{\circ}, & 5 \leq t<10 \mathrm{~s} \\ \theta_{r}=15^{\circ}, \psi_{r}=45^{\circ}, \gamma_{r}=-10^{\circ}, & 10 \leq t<15 \mathrm{~s} \\ \theta_{r}=0^{\circ}, \psi_{r}=0^{\circ}, \gamma_{r}=0^{\circ}, & 15 \leq t<20 \mathrm{~s} \\ \theta_{r}=-15^{\circ}, \psi_{r}=-45^{\circ}, \gamma_{r}=10^{\circ}, & 20 \leq t \leq 24 \mathrm{~s}\end{cases}
$$

The gains of the PD controller, $K_{p}$ and $K_{d}$ are taken as to 8 and 4 , respectively. Besides, in order to eliminate the chattering effect of the SMC, a function $\operatorname{sgn}\left(u_{p d}\right)=u_{p d} /\left(\left|u_{p d}\right|+\delta\right)$ is 
used to replace the traditional signum function in aforementioned update rules. Here, $\delta$ is set to be 0.05 .

Fig. 4.4 and 4.5 compare the tracking performances of the proposed control approaches with a PD controller working alone when sinusoidal attitude signals are chosen as references. In Fig. 4.4 a T2FNN using triangular MFs with uncertain width is employed to work in parallel with a PD controller. As shown in this figure, a PD controller could bound the error signals in the neighbourhood of zero, however, it cannot eliminate the steady errors (See Fig. 4.4a and 4.4c). In contrast, the proposed method integrated a PD controller and a T2FNN together can definitely remove the unneeded steady state error and efficiently track the desired signals. An extremely similar finding appears in Fig. 4.5. The main difference with the Fig. 4.4 is that, the triangular MFs embedded in the T2FNN are with variable centres. Using this control approach, the steady state error could also be eliminated, compared to the result led by using the PD controller alone.

Besides, tests to track step reference signals are also carried out in order to further verify the reliability and robustness of the two methods proposed before. Test results are shown in both Figs. 4.6 and 4.7 It can be seen from these two figures, the proposed control frame surpasses the solo PD controller in performance for this case of step signal inputs. Similar to the previous simulations, these two proposed control methods can eliminate the steady state error while only using a PD controller cannot.

\subsection{Conclusion}

In this chapter, a T2FNN controller has been proposed for the attitude control of a FMWAV with uncertainties and disturbances. To deal with those uncertainties and disturbances, two kind of type-2 triangular MFs (i.e., with uncertain width and center, respectively) are merged into the T2FNN. In addition, SMC theory-based learning algorithms is employed to adapt parameters in both antecedents and consequents of the T2FNN. Next, the stability of the learning algorithms is analysed. The simulation results show that the proposed control frame, in which adaptive T2FNN works in parallel with a conventional PD controller, can control the system effectively and it outperforms conventional PD controller used alone. It is also proved that the use of T2FNN with the two different type-2 triangular MFs could effectively cope with system uncertainties and environmental disturbances. 


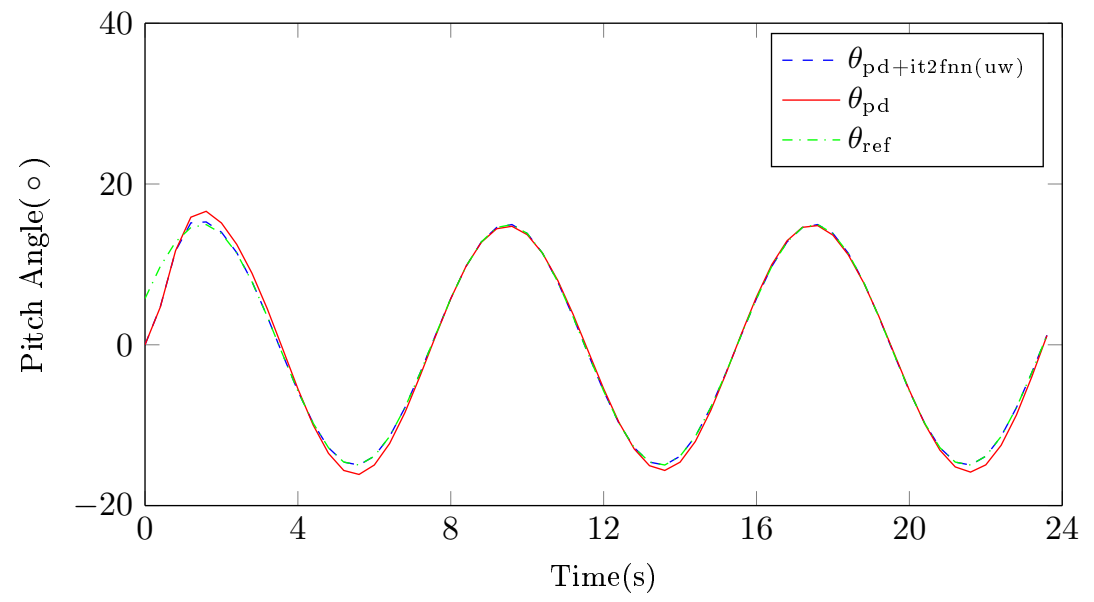

(a)

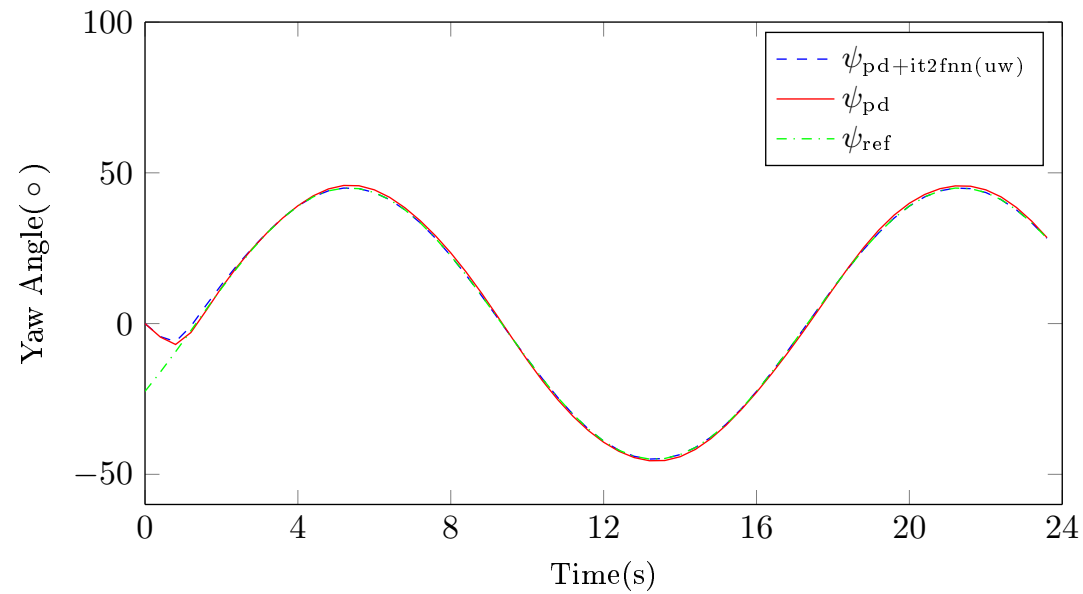

(b)

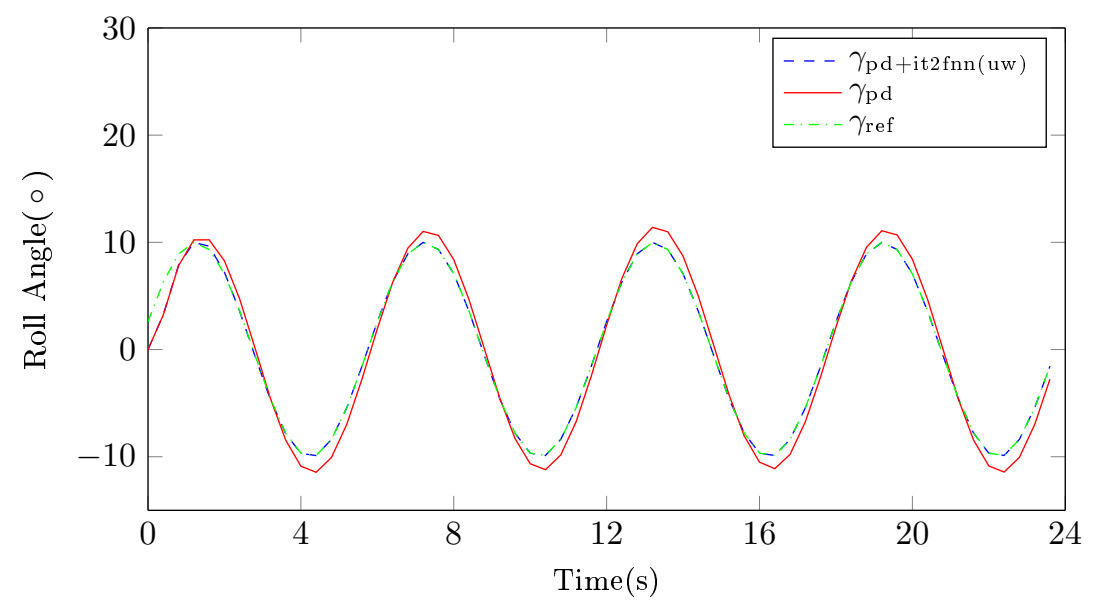

(c)

Figure 4.4: Attitude control responses of a FWMAV in which a T2FNN using triangular MFs with uncertain width (UW) when tracking the sinusoidal reference signals. (a) Pitch response. (b) Yaw response. (c) Roll response. 


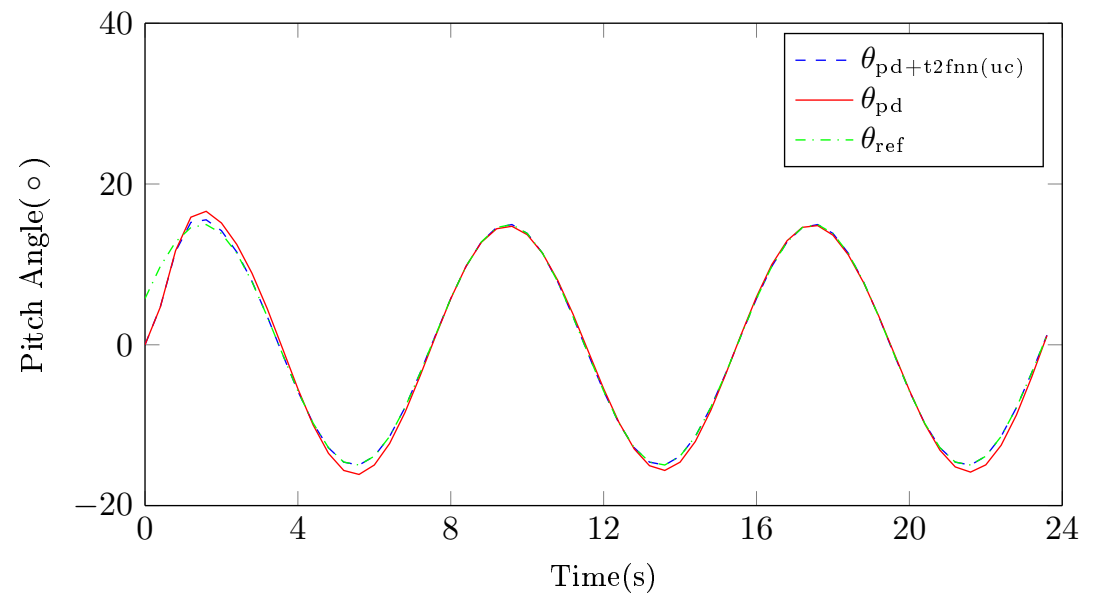

(a)

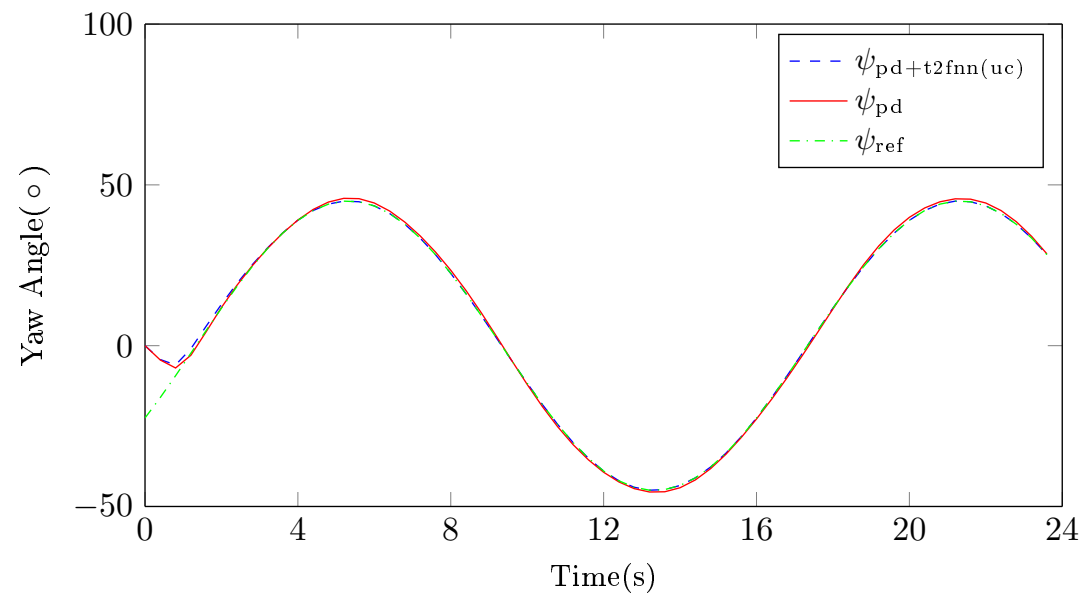

(b)

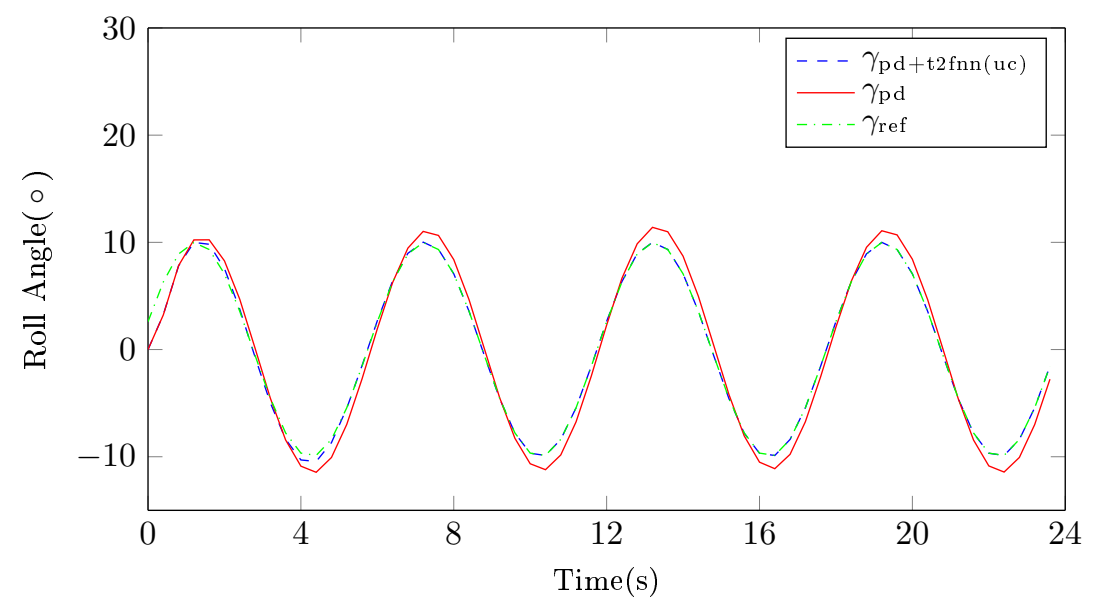

(c)

Figure 4.5: Attitude control responses of a FWMAV in which a T2FNN using triangular MFs with uncertain center (UC) when tracking the sinusoidal reference signals. (a) Pitch response. (b) Yaw response. (c) Roll response. 


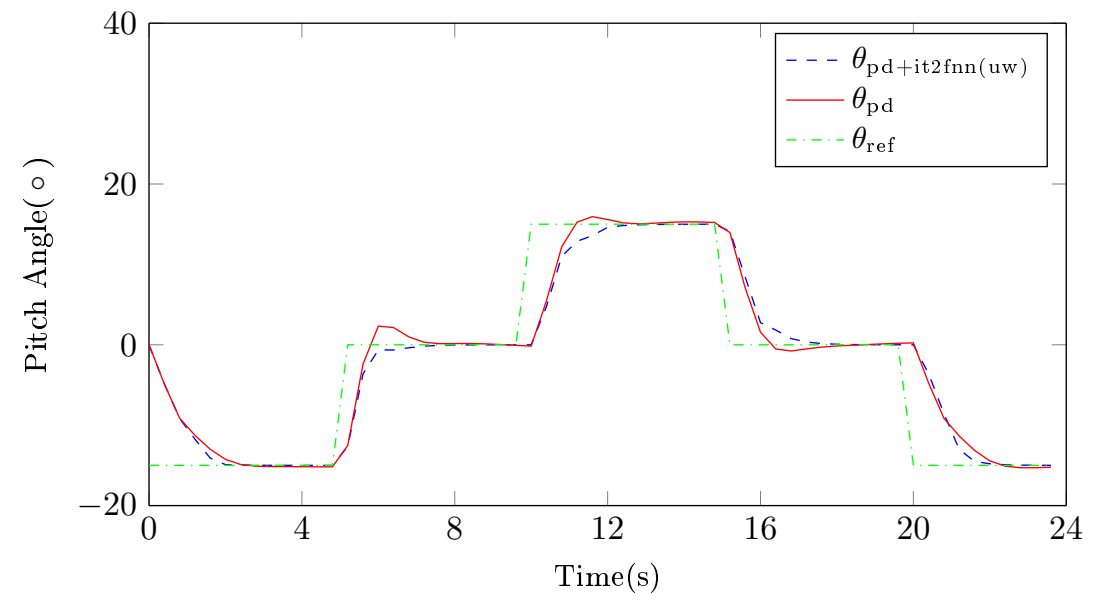

(a)

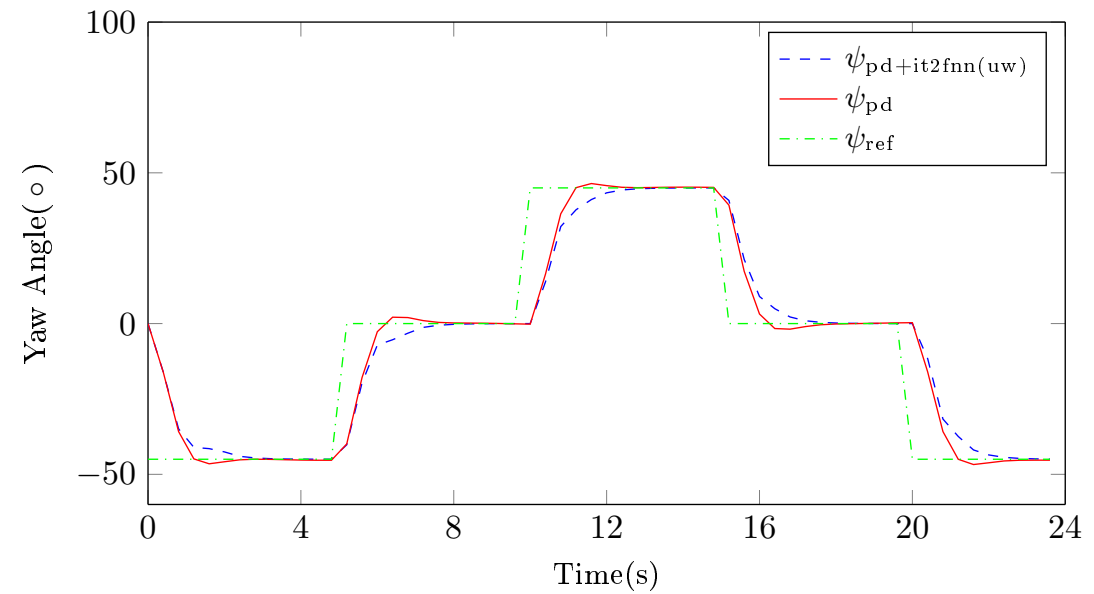

(b)

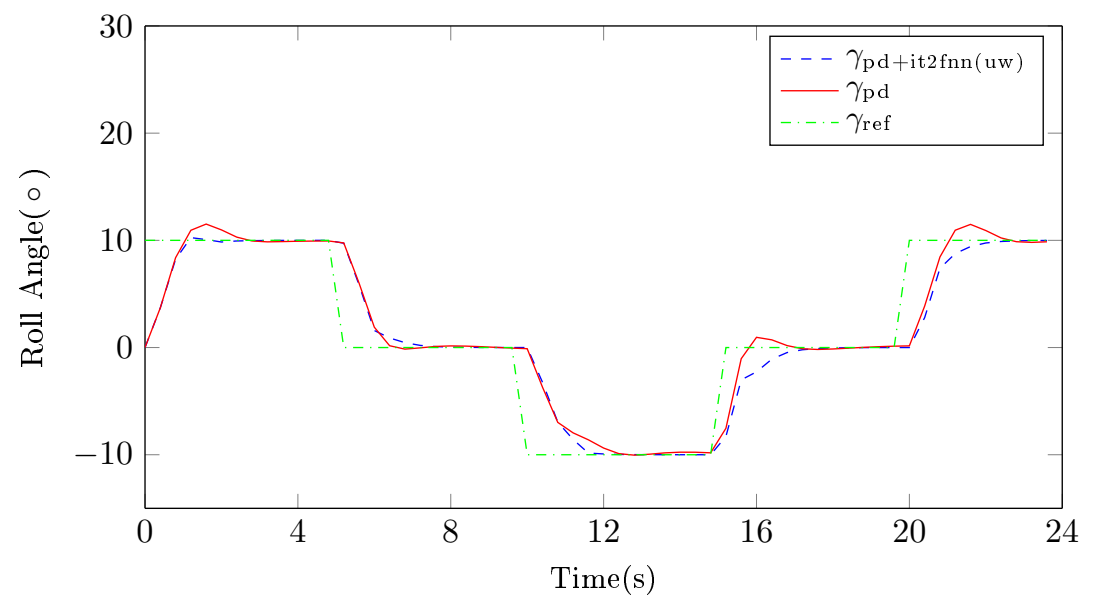

(c)

Figure 4.6: Attitude control responses of a FWMAV in which a T2FNN using triangular MFs with uncertain width (UW) when tracking the step reference signals. (a) Pitch response. (b) Yaw response. (c) Roll response. 


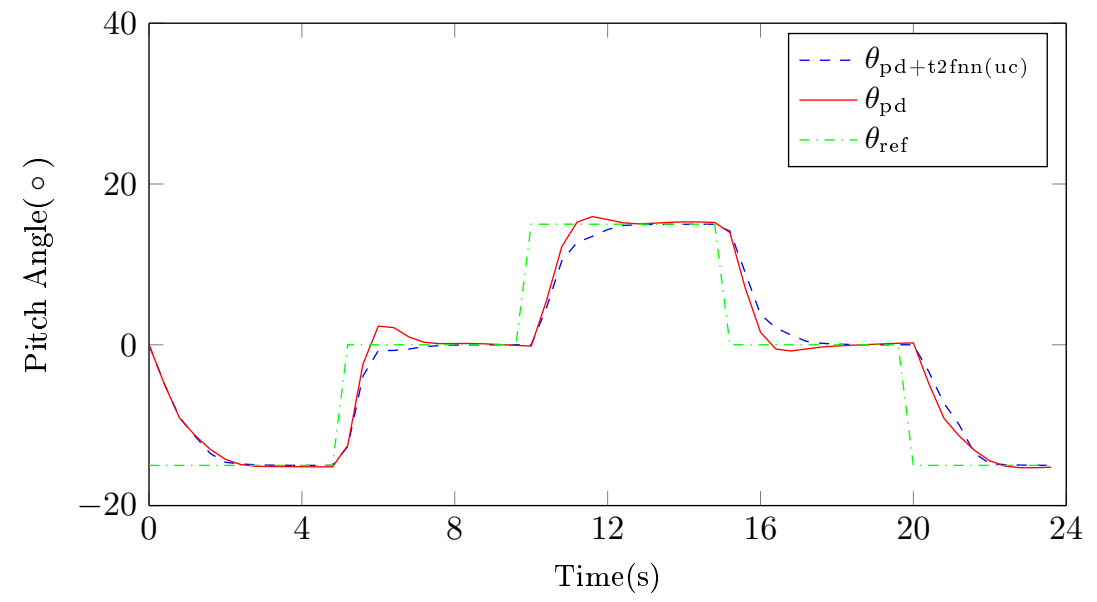

(a)

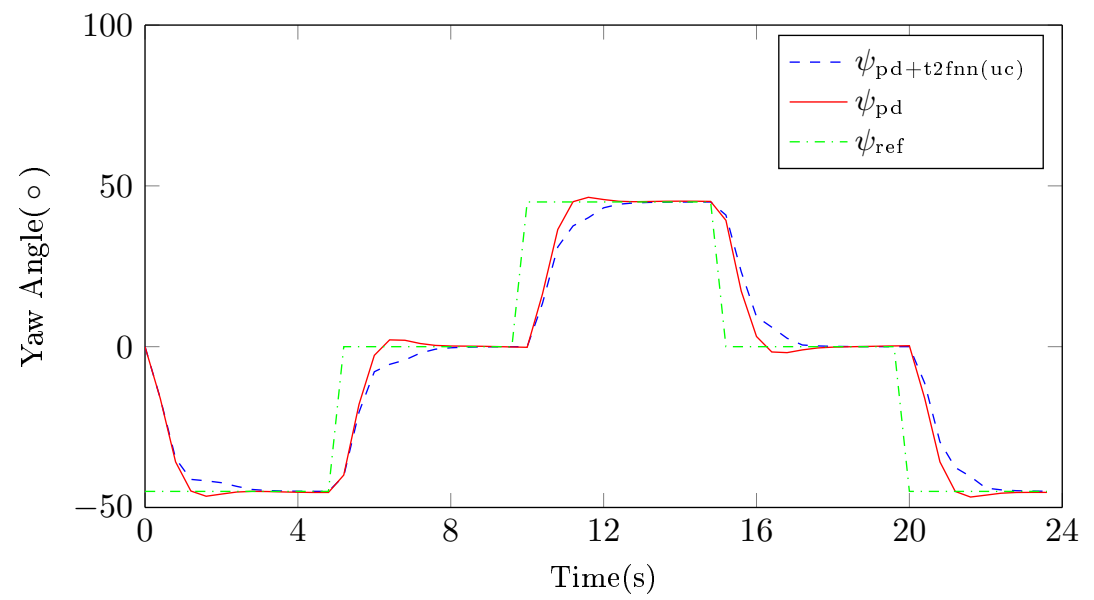

(b)

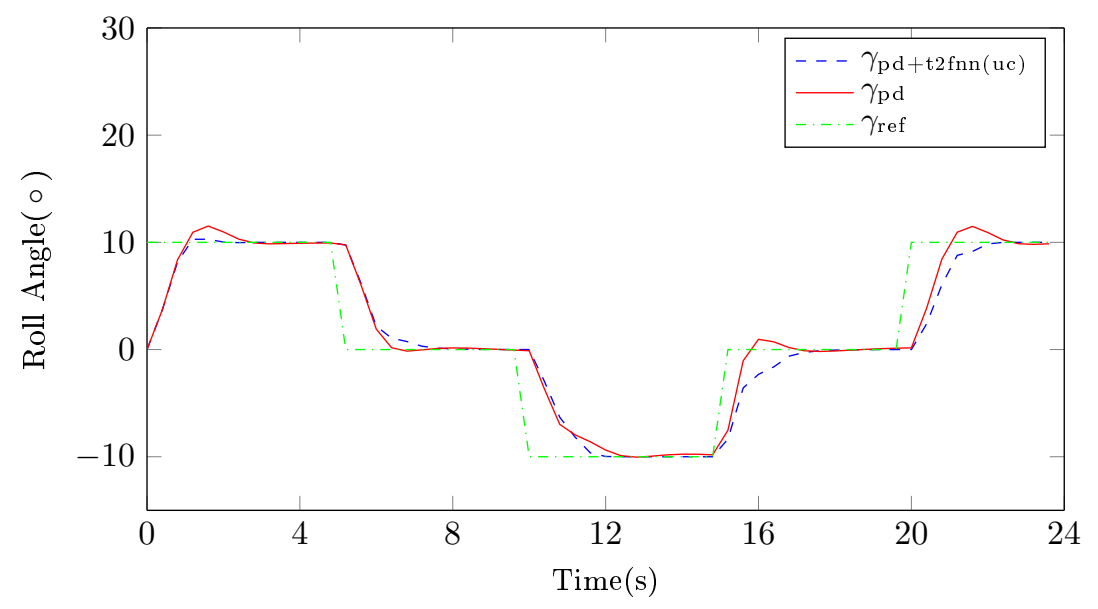

(c)

Figure 4.7: Attitude control responses of a FWMAV in which a T2FNN using triangular MFs with uncertain center (UC) when tracking the step reference signals. (a) Pitch response. (b) Yaw response. (c) Roll response. 


\section{5}

\section{Design and Minor Tests of a Morphing-wing Structure}

\subsection{Introduction}

In nature, there are a large number of morphing-wing designs whose planform, camber or twist can continuously change, such as wings of insects, birds and bats (71). Distinctly from flapping birds and insects, bats are provided with much more complex wing structures. Bat wings consist of more than two dozens of independently controlled joints, which provide them with the capability to positively adjust the wing morphing according to the aerodynamic demands in flight (27). Additionally, the high accelerations generated by inertial forces of massive wings can enhance their own maneuverability (184, 185, 186). Taking advantage of such forces, bats can perform roll and pitch maneuvers by solely controlling wings geometry, without the need of appendices that need aerodynamic loads (i.e. speed). This could dramatically improve MAVs manoeuvring at low speed, and therefore allow operating in confined spaces. Thus, bats are attractive models to study and mimic in order to improve flight performance of current MAVs.

In earlier work (40, 58, 145), we presented a robot, the BatBot, based on a bat-wing flapper done by Bahlman et al. (38). The BatBot used a servo motor to drive flapping motions while morphing-wing actions were actuated by SMAs. However, this prototype had a very low flapping frequency due to the mechanical limitations of its flapping mechanism. Furthermore, due to relatively small tension forces produced by SMAs, the morphing actions of wings were limited. Considering such issues, a new morphing-wing bat-like robot was designed and built.

At the beginning of Chapter 3, we have described a first compliant flapping mechanism 
Table 5.1: Sizes of Wing Bone Components

\begin{tabular}{c||c||c||c}
\hline Skeleton & Scales[Unit] & Skeleton & Scales[Unit] \\
\hline \hline Shoulder & $52.00 \mathrm{~mm}$ & Digit III & $105.25 \mathrm{~mm}$ \\
\hline Humerus I & $36.20 \mathrm{~mm}$ & Digit IV & $83.75 \mathrm{~mm}$ \\
\hline Humerus II & $42.00 \mathrm{~mm}$ & Digit V & $85.75 \mathrm{~mm}$ \\
\hline Radioulna I & $72.50 \mathrm{~mm}$ & Rib & $59.50 \mathrm{~mm}$ \\
\hline Radioulna II & $93.50 \mathrm{~mm}$ & & \\
\hline
\end{tabular}

that was introduced in order to allow higher flapping frequencies. Such mechanism has been employed to drive flapping motions of the robot described here.

In this chapter, two additional aspects of contents are presented. First of all, the fabrication of a novel morphing-wing structure is introduced. Secondly, experimental tests are presented, whose purpose is to measure the whole body lift and thrust forces generated by two symmetrical wings and the roll moments when the shapes of the two wings are different.

\subsection{Fabrication, Assembly and Actuator}

The robotic skeletal framework is composed of eighteen rigid elements, mimicking the bat's wing structure: body, rib, shoulder, humerus, radioulna, digits III, digits IV and digit V, and tail. These components are shown in Fig. 5.1, and their dimensions are shown in Table 5.1. The wings had two DoFs: the shoulders actuated by the crank-rocker mechanism could move up and down, therefore to realize flapping-wing motions. The movements of morphing wings were driven by motions of sliders in the middle of the body. At the current stage of development, they are operated manually.

All mechanical parts were made of ABS plastic printed using 3D printer. The wing membrane is made of polytene. The fully assembled model, displayed in Fig. 5.1, had a wing span of $22.9 \mathrm{~cm}$ and a wing area of $233.6 \mathrm{~cm}^{2}$ when wings completely unfolded. The average chord is $9.73 \mathrm{~cm}$. When wings are fully folded, wingspan was decreased by $23 \%$ and wing area was reduced by $35 \%$. The key features of the morphing-wing flying robot are summarised in Table 5.2 The flapping-wing actuator adopted is a miniature gear motor with dimensions $23.9 \mathrm{~mm} \mathrm{x}$ $9.9 \mathrm{~mm} \times 11.9 \mathrm{~mm}$, high-power brushed DC motor whose mass is $11 \mathrm{~g}$. 


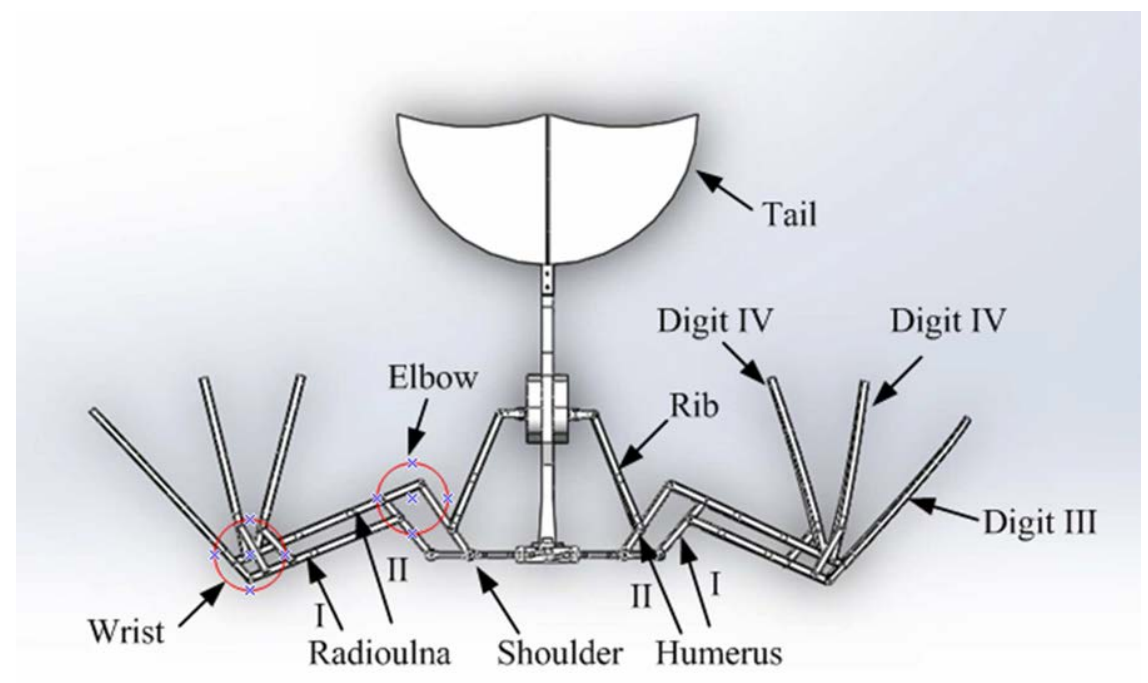

Figure 5.1: The whole body of a flying robot

Table 5.2: Key parameters of the morphing-wing flying robot

\begin{tabular}{c||c}
\hline Parameters & Scales [Unit] \\
\hline \hline Wing Span & $22.9[\mathrm{~cm}]$ \\
\hline Single Wing Area & $233.6\left[\mathrm{~cm}^{2}\right]$ \\
\hline Average Chord & $9.73[\mathrm{~cm}]$ \\
\hline Flapping-wing Angle & $55^{\circ}$ \\
\hline Maximum Flapping Frequency & $3.5[\mathrm{~Hz}]$ \\
\hline Maximum Wing Span Reduction & $23 \%$ \\
\hline Maximum Wing Area Reduction & $35 \%$ \\
\hline Dhole Motor Weight & $11 \mathrm{~g}$ \\
\hline
\end{tabular}

\subsection{Experiments}

The model has been successfully tested flapping up to $3.5 \mathrm{~Hz}$, which was $0.5 \mathrm{~Hz}$ lower than the frequency used in the simulation (to avoid risk of breaking the mechanism). To test the prototype and measure lift, thrust and roll moment produced by the prototype, it was mounted on a 3-DoF test platform, shown in Fig. 5.2. The robot was fixed on a piezoelectric force sensor to measure the dynamic load generated when operating. Voltage data caused by the load cell 


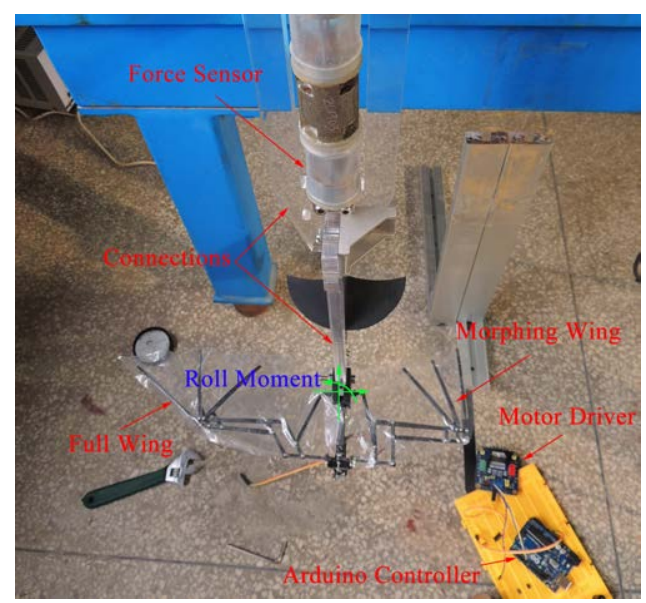

Figure 5.2: Force test platform of the flying robot

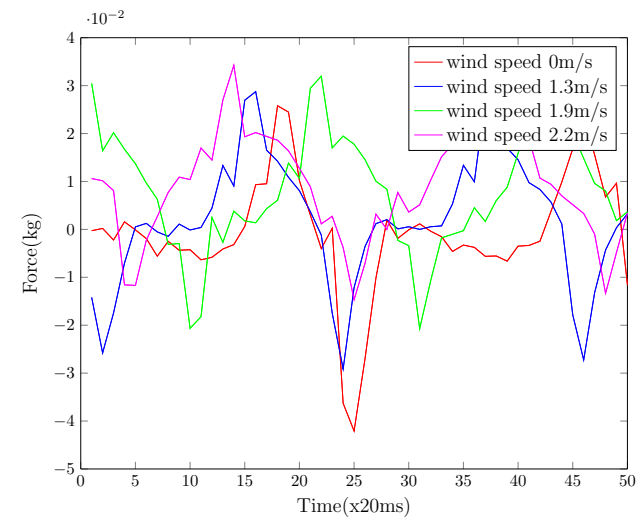

Figure 5.3: Lift forces generated by full wings at $\mathrm{AoA}=23^{\circ}$

was transformed into a digital signal. Data was sampled at a rate of $2000 \mathrm{~Hz}$ by using National Instruments NI USB 6009 DAQ and was filtered and scaled by using LabView software for their analysis. All the experiments were done in collaboration with Northwestern Polytechnical University, China. The test platform was test in a wind tunnel measuring $105 \mathrm{~cm}$ in length and $45 \mathrm{~cm}$ by $65 \mathrm{~cm}$ in the test cross-section. Air flow was generated by an air blower with variable speed control.

Fig. 5.3 and 5.4 show how lift forces and thrust forces produced by full wings (without morphing) at the angle of attack (AoA) $23^{\circ}$ vary with wind speeds. Lift forces increases when the wind speed increases, while drag forces decrease with the increase of wind speed.

Figures 5.5, 5.6 and 5.7 show how the roll moment changes of a single morphing-wing robot shown in Fig 5.2 at different AoAs (i.e. $13^{\circ}, 18^{\circ}, 23^{\circ}$ ) for two different flapping speeds. 


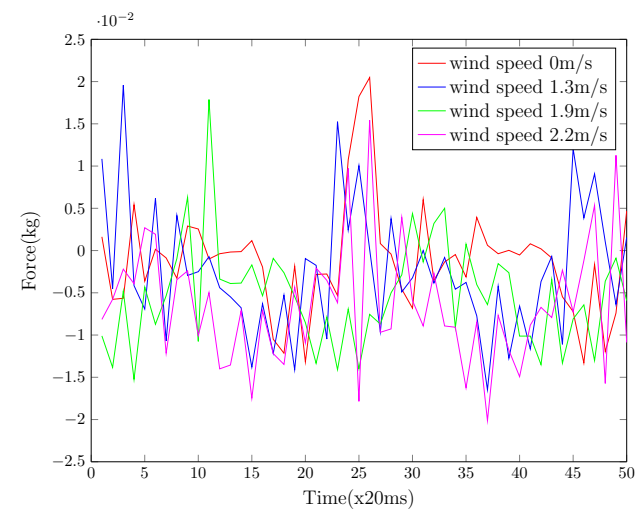

Figure 5.4: Thrust forces generated by full wings at $\mathrm{AoA}=23^{\circ}$

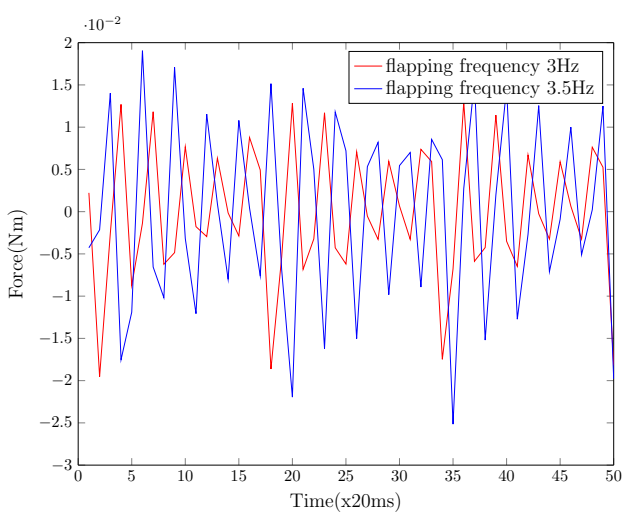

Figure 5.5: Roll moment generated by single morphing wing at $\mathrm{AoA}=13^{\circ}$

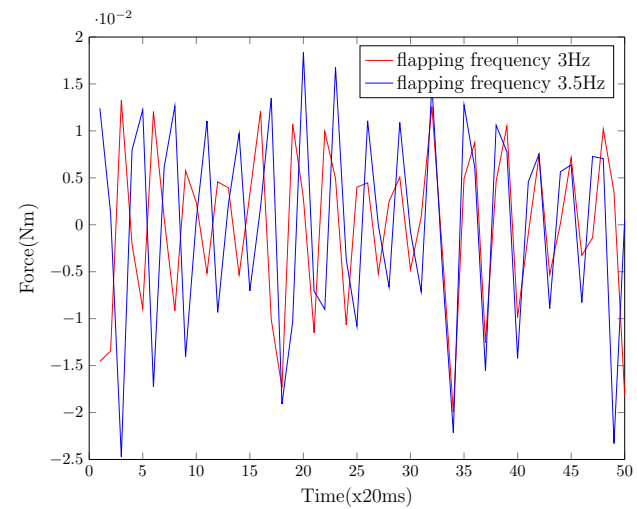

Figure 5.6: Roll moment generated by a single morphing wing at $\mathrm{Ao} A=18^{\circ}$

These experiments confirmed that a roll moment is actually generated when one of the wings is folded and the other is extended. Such a roll moment can be controlled by controlling 


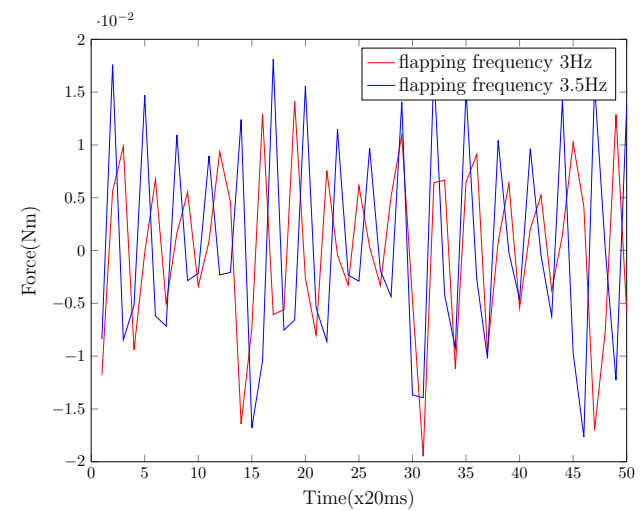

Figure 5.7: Roll moment generated by a single morphing wing at $\mathrm{AoA}=23^{\circ}$

the wings' geometries and hence the prototype can be manoeuvred by a flight controller acting on the morphing wings. Also, note that the roll moment increases when flapping frequency increases.

\subsection{Conclusion}

In this study we built and characterized a morphing-wing flying robot. Inspired by real bats, we are developing the idea that by properly controlling the modulation of the wing geometry, more efficient flight can be achieved, just as bats efficiently generate net body forces taking advantage of their large wing-to-body mass ratio. Moreover, we are investigating how roll moments can be generated by actively controlling wings' geometry, without the need appendices that need aerodynamic loads (i.e. speed) and can thus improve manoeuvring at low speed. This approach opens the path to the development of novel attitude controllers (flight control) applied to flapping MAV, where the inertial forces produced by the wings are owed for the production of net body accelerations. This possibility has great potential to improve MAV's capability to operate in confined spaces.

Future work will be devoted to improving the design to obtain bigger roll moments and to include pitch motion (by means of controlling the shoulders' position). Moreover, lightweight piezomotors will be used to control the wings' geometry, and the prototype will be equipped with on-board computation capabilities for flapping and morphing control. In addition, whether the morphing-wing robot can improve the energy efficiency compared to a fixed-wing robot is also taken into consideration and is carried out in the experiments in the future. 


\section{6 \\ Conclusions and Open Challenges}

\subsection{Conclusions}

This dissertation investigates biological designs learning from thorax structures of insects and bats' wings and flight control in order to gain a better understanding for the development of flapping wing micro air vehicles. The main contributions of this thesis includes three aspects:

\section{Optimization Design}

The number of literatures on FWMAVs and their designs of flapping mechanisms is fairly big, however, very little attention has been given to the details of modelling and optimization design. In this thesis, we propose two methods for designing and analysing the behaviours of compliant transmission mechanisms for FWMAVs. Compared to other optimization methods proposed (see, e.g. (4, 147)), our methods are suitable for pseudo-rigid models since internal interaction forces do not need to be considered. Furthermore, the elastic potential energy stored in the compliant joints can be easily taken into account. The two methods can be generalized to other compliant transmission mechanisms and constitute, therefore, a significant contribution to the field of FWMAVs. All this work is summarized in the Design and Optimization Methodology for Compliant Flapping Mechanisms described in algorithm 2 in Chapter 3.

\section{Flight Control}

In this thesis, we proposed a type-2 fuzzy neural network working in parallel with a derivative differential controller to cope with system uncertainties and environmental disturbances. Two new online learning algorithms were employed to update the parameters in both antecedents 
and consequents of the fuzzy neural network. In addition, we have also proved the stabilities of the two methods by using both Lyapunov and sliding mode control theories. Final simulation results demonstrate that both the two methods are effective and reliable in the attitude control of a FWMAV under the conditions with system uncertainties and environmental disturbances. Keep in mind that control methods with an auxiliary term are only suited for second order nonlinear dynamic systems (as pointed out in (165)) while these two methods are applied to other control systems..

\section{Morphing-wing structure}

Bats have shown excellent flight characteristics in terms of high maneuverability and good stability by varying the morphological shapes of wings. We believe that mimicking the behaviours of bats' wings is a promising way to design more efficient flapping-wing MAVs. In this case, a morphing-wing structure that mimics the mechanical system of bats' wings was integrated into the design of a flapping-wing robot. To explore the influences of changing the wing shape on the flight performance of the robot, in terms of lift and thrust forces, several experiments were performed. This approach could provide an insight for developing a morphing-wing FWMAV in future, with purpose of finally improving the maneuverabilities of current FWMAVs. These development is a must towards achieving the first bat-like robot capable of sustained autonomous flight.

\subsection{Open Challenges}

We conclude this chapter with a review of open challenges that can constitute the bases for future works in this field.

\section{- Complaint transmission mechanisms: design, fabrication and control}

One of the biggest challenges is the difficulty of analysing and designing compliant transmission mechanisms. Finite element methods are widely used to analyse compliant mechanisms. Current commercial software has the capability of analysing the large, non-linear deflections often associated with compliant mechanisms. The general nature of the method makes it applicable for a wide range of geometries, materials and applications. Topology optimization is often integrated with finite element methods to consider many possible ways of distributing material with the design domain (187). This has the potential to find better designs that would be beneficial for flapping transmission mechanisms. In 
the previous section, we have proposed two methodologies for design and optimization of CTMs, which we believe is useful for future designs of FWMAVs.

As for the manufacturing of such small systems, much progresses have been made in advanced fabrication techniques like laser micro machining (50), surface micro machining (51), and polymer micro machining (52). The latest advances in 3D printing technology allow manufacturing components with the needed mechanical characteristics and printing resolution that can speed up the development of FWMAVs. Using the 3D printing technology, the structure arrangement of the compliant joints will be isotopic rather than homogenous (188). Hence, new theoretical models and modelling approach will be needed to predict the stiffness characteristic of the compliant structure. The advancement in material science will be crucial since this advanced technology requires new material that can be actuated while providing necessary strength and stiffness to the overall structural integrity. Most importantly, the new material engineered must have high endurance limit and predictable fatigue life.

Furthermore, the control of compliant transmission mechanisms for flight is a key topic that hardly appear in the literature. Recently, Kern et al. (189) proposed a robust method to control compliant mechanisms with large deflections. In their work, flexure hinges were equivalent to pseudo rigid-body systems with uncertainty. Then, by synthesizing current available theories of multi-body dynamics, a robust controller was employed to control a leaf-spring-type flexure hinge to deflect. Similar ideas appeared in the control of compliant-mechanism-based manipulators, where sliding control (190) and feedback linearisation (191) were adopted for positioning of the manipulator, respectively. These approaches could provide us some inspirations for controlling CTMs of FWMAVs. Combining the aforementioned analysis and design approaches of compliant mechanisms, existing multi-body dynamic theories and control methodologies will be essential for the control of compliant transmission mechanisms. The precise position control of CTMs is also one of the most important issues that need to be solved for guaranteeing CTMs produce the desired trajectories and motions. This is a similar problem as in compliant manipulators (190, 191), so this field may provide good literature for CTMs position control.

- Compliant actuators: batteries, on-board power electronics, and selection 
Currently, one of the main limitations of compliant actuators is the power system (including batteries and on-board power microelectronics), especially for smart actuators-based transmission systems used for FWMAVs described in Section 2.2.3. As described before, for most smart actuators, except electromagnetic ones, high voltages are needed in order to produce sufficient forces or displacements. Unfortunately, current compact energy sources suitable for flapping-wing micro robots, like conventional batteries, ultracapacitors (192), solar cells (193) and fuel cells (194), only generate output voltages below 5 Volts. Connecting such elements in series does not seem to be practical due to packaging overhead (18). Lithium polymer batteries are the only commercially available technology that can satisfy the requirements of flapping MAVs at present. As new technologies of batteries are emerging, including micro solid oxide fuel cells (195), lithium batteries with silicon nanowire anodes (196), and lithium air batteries (197), the battery problems faced by smart actuators, perhaps, could be solved in the future.

Considering the current voltage problems of batteries, some researchers attempted to design the interfaces of micro power electronics that are capable of converting a low input voltage from an energy source into a time-varying, high-voltage drive signal for powering smart actuators. Karpelson et al. (198) presented a sub-100mg high-voltage power electronic converter for piezoelectric microrobots. After optimization, the converter could output voltages of $200 \mathrm{~V}$ at a $3.7 \mathrm{~V}$ input voltage, typical of lithium polymer batteries. The applicability of the drive circuits was verified on a flapping wing robotic insect. Some other attempts of electric interfaces for piezoelectric actuators could be found in (199, 200, 201). As for DEAs, Chen et al. (202) proposed a power electronic interface with light weight, high power density and high step-up conversion ratio, for driving bimorph dielectric elastomer actuators at low frequency. Through their experimental validations, the dual-stage topological circuit could drive a $600 \mathrm{~V}$ DEA at $4 \mathrm{~Hz}$. Even through the circuit could drive DEAs at low frequency, the high operation voltage of $600 \mathrm{~V}$ is still a huge obstacle that prevents DEAs from widely applying to micro flying vehicles.

Besides the common power problem for compliant actuators, limitations and potential performance trade-offs for each kind of actuator are also required to consider. Piezoelectric actuators have a limited travel range and non-linear displacement characteristics, but they can deliver very high driving force and bandwidth, which makes them potential selections for micro- to macro- scale compliant actuators. As the size increases from 
macro-scale onwards, electromagnetic actuation can be an alternate solution to the piezoelectric actuators. In general, electromagnetic actuators adopt two types of techniques, i.e., the solenoid actuation and the Lorentz-force actuation, to realize the driving motion. Solenoid actuators may have the advantage of large force-to-size ratio while the Lorentz-force actuator has linear characteristic and large travelling range. Comparing to piezoelectric actuators, a disadvantage of electromagnetic actuators is that, they lack the capability of providing an additional non-actuation stiffness when power-off. As for DEAs, the low stiffness of dielectric materials is a significant design challenge for actuation and power generation for FWMAVs. Much more information on the actuator selection, power electronics topologies and circuit component fabrication options can be referred in (93, 203).

\section{- Design, dynamic modelling and control of morphing wings.}

In nature, flying animals are capable of maneuvering using flexible wings, which can deform significantly with effects of both the driving motion of the structure and the aerodynamic loading of the fluid surrounding wings. Given the flight characteristics of wings, passive morphing aerodynamic structures were brought into the fabrication of wings for FWMAVs. At present, the passive morphing wings adopted in FWMAVs are without the capability of active adjustments. In such case, how to make compliant wings produce synchronous movements under complex and unstable airflow environment and thereby generate stable forces for fight is a big challenge for research. In addition, active morphing-wing concepts was proposed by researchers (see, e.g., $(38,40,58,204,205)$ ) for improving efficiency and manoeuvrability. However, the accompanying problems associated with actively morphing wings, e.g., much more complex morphing-wing structures, have not been solved yet.

Dynamic modelling and control represents another big challenge for morphing-wing FWMAVs. Currently, most researches on dynamic modelling of insect-scale (55, 56) or birdscale FWMAVs(57) are mainly through first-principles modelling (54). In their models, each of wing has only one flapping DoF and the mass of wing is too small to be neglected while modelling. However, purely using this kind of modelling methodologies is not sufficient to solve the counterpart of a morphing-wing FWMAV due to their complex motion on wings. Meanwhile, the mass of the morphing-wing structure can not be ig- 
nored any more. The subsequent shift of the center of mass $(\mathrm{CoM})$ should be taken into consideration while morphing.

Due to the great difficulties faced in the dynamic modelling for morphing-wing FWMAVs, very few research on flight control for morphing-wing FWMAVs has been reported. Recently, Ramezani et al (205) proposed a nonlinear controller based on the Tikhonov's singular perturbation theory for a bat-inspired flapping aerial vehicle. The objective of the controller is to track desired attitude angles of the morphing FWMAV. However, such a controller has not been tested in sustained untethered flight yet. Colorado et al. (206) proposed a flight controller using a backstepping control strategy, extended with an integral action named desired angular acceleration function (DAF) to produce desired roll and pitch angular accelerations. Even though wind tunnel experiments were also made to assess the effectiveness of the proposed controller and to quantify the roll and pitch manoeuvers generated, the real performance of the controller during outdoor flight needs to be further verified. 


\section{7}

\section{Publications}

The development of this thesis has allowed the following scientific production, including JCR referred journals, conferences proceedings and other dissemination activities.

\section{Peer-reviewed journals articles}

1. C. Zhang, C. Rossi, 2016. A Review of Compliant Transmission Mechanisms for Bio-inspired Flappingwing Micro Air Vehicles. Bioinspiration and Biomimetics (Q1 JCR).

2. J. Colorado, C. Rossi, C. Zhang and A. Barrientos, 2015. Towards efficient flight: insights on proper morphing-wing modulation in a bat-like robot. Advanced Robotics, vol. 29, no. 24, pp. 1599-1610, published by Taylor \& Francis. DOI: 10.1080/01691864.2015.1082501. (Q2 SCOPUS; Q4 JCR)

\section{Peer-reviewed conference papers}

1. C. Zhang, C. Rossi, W. He and J. Colorado, 2016. Virtual-work-based Optimization Design on Compliant Transmission Mechanism for Flapping-wing Aerial Vehicles. 1st International Conference on Manipulation, Automation and Robotics at Small Scales (MARSS), Paris, France, Jul. 18-22, 2016.

2. C. Zhang, C. Rossi and D. Song, 2015. Design and Fabrication of a Novel Morphing Wing Flapping MAV. 2nd Colombian Conference on Automated Control (CCAC), Manizales, Colombia, Oct. 14-16, 2015.

3. C. Rossi, Z. Yuan, C. Zhang, A. Barrientos and W. Coral, 2014. Shape memory alloy-based High Phase Order Motor. 11th International Conference on Informatics in Control, Automation and Robotics (ICINCO), Vienna, Austria, Sep. 1-3, 2014.

\section{Other}

1. C. Zhang, C. Rossi, J. Colorado, The BatBot, Robot Safari exposition, Science Museum London, November 2013 


\section{References}

[1] B.J. TSai and Y.C. FU. Design and aerodynamic analysis of a flapping-wing micro aerial vehicle. Aerospace Science and Technology, vol.13, no.7, pp.383-392, 2009. vi 3

[2] J.W. Gerdes, S.K. Gupta, and S.A. Wilkerson. A review of birdinspired flapping wing miniature air vehicle designs. inspired flapping wing miniature air vehicle designs.
Journal of Mechanisms and Robotics, vol.4, no.2, pp.021003, 2012. vi 2311

[3] C.K. Hsu, J. Evans, S. Vytla, and P. Huang. Development of flapping wing micro air vehicles-design, CFD, experiment and actual flight. In 48th AIAA Aerospace Sciences Meeting, pp.4-7, 2010. vi 3

[4] R. Madangopal, Z.A. Khan, and S.K. Agrawal. Biologically inspired design of small flapping wing air vehicles using four-bar mechanisms and quasi-steady aerodynamics. Journal of Mechanical Design, vol.127, no.4, pp.809-816, 2005. vi

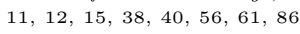

[5] Z.A. Khan and S.K. Agrawal. Design and optimization of a biologically inspired flapping mechanism for flapping wing micro air vehicles. In IEEE International Conference on Robotics and Automation (ICRA), pp.373-378, 2007. vi 11$] 12$

[6] S.S. BAEK, K.Y. MA, AND R.S. FEARING. Efficient resonant drive of flapping-wing robots. In IEEE/RSJ International Conference on Intelligent Robots and Systems(IROS), pp.2854-2860, 2009. vi 12151640

[7] D. Azhar, M.and Campolo, G.K. Lav, L. Hines, and M. Sitti. Flapping wings via direct-driving by DC motors. In ping wings via direct-driving by DC motors. In IEEE International Conference on Rob
pp.1397-1402, 2013. vi $12 \quad 13 \quad 40$

[8] L. Hines, D. Campolo, and M. Sitti. Liftoff of a motordriven, flapping-wing microaerial vehicle capable of resonance. IEEE Transactions on Robotics, vol.30, no.1, pp.220232, 2014. vi $1 2 \longdiv { 1 3 } 1 5,40$

[9] W. Bejgerowski, A. Ananthanarayanan, D. Mueller, and S.K. Gupta, Integrated product and process design for a flapping wing drive mechanism. Journal of Mechanical Design, vol.131, no. $6, p p .061006,2009$. vi $13 \lcm{15} 41$

[10] D. Mueller, H.A. BRuck, ANd S.K. Gupta. Measurement of thrust and lift forces associated with drag of compliant flappin test stand design. Experimental Mechanics, vol.50, no.6, pp.725735, 2010. vi 131541

[11] W. Bejgerowski, J.W. Gerdes, S.K. Gupta, and H.A. Bruck. Design and fabrication of miniature compliant hinges for multi-material compliant mechanisms. International Journal of Advanced Manufacturing Technology, vol.57, no.5-8, pp.437 452, 2011. vi 131415

[12] G.K. LaU, Y.W. Chin, J. T.W. GoH, AND R.J. Wood. Dipteraninsect-inspired thoracic mechanism with nonlinear stiffness to save inertial power of flapping-wing flight IEEE Transactions on Robotics, vol.30, no.5, pp.1187-1197, 2014 vi $13,14,15,41$

[13] R. SAHA K. C. Gauoway, aNd R. J. Wood Elastic element integration for improved flapping-wing micro air vehicle performance. IEEE Transactions on Robotics, vol.29, no.1, pp.32-41, 2013. vii $14 \quad 15415859$

[14] R. Sahai, K.C. Galloway, M. Karpelson, and R.J. Wood. A flapping-wing micro air vehicle with interchangeable parts for system integration studies. In IEEE/RSJ International Conference on Intelligent Robots and Systems (IROS), pp.501-506, 2012. vii 14 15

[15] K. Mateti, R. Byrne-Dugan, C.D. Rahn, S. Tadigadapa, et al. Monolithic SUEX Flapping Wing Mechanisms for Pico Air
Vehicle Applications. Journal of Microelectromechanical SysVehicle Applications. Journal of Microelectromechanical Sy
tems, vol.22, no.3, pp.527-535, 2013. vii 2016]17] 18

[16] K. Mateti, R.A. Byrne-Dugan, S.A. Tadigadapa, and C.D. Rahn. Wing rotation and lift in SUEX flapping wing mechanisms. Smart Materials and Structures, vol.22, no.1, pp.014006, 2012. vii $1 7 \longdiv { 1 8 } 2 3$

[17] V. Arabagi, L. Hines, and M. Sitti. Design and manufacturing of a controllable miniature flapping wing robotic platform. International Journal of Robotics Research, pp.0278364911434368, 2012. vii $17 \quad 18,23$

[18] M. Karpelson, G.Y. Wei, and R.J. Wood. Driving high voltage piezoelectric actuators in microrobotic applications. Sensors and Actuators A: Physical, vol.176, pp.78-89, 2012. vii $1 7 \longdiv { 1 9 } 2 3 8 9$

[19] R.J. Wood. The first takeoff of a biologically inspired at-scale robotic insect. IEEE Transactions on Robotics, vol.24. no.2, pp.341-347, 2008. vii 17,1923

[20] N.O. Pérez-Arancibia, K.Y. Ma, K.C. Galloway, J.D. Greenderg, and R.J. Wood. First controlled vertical flight of a biologically inspired microrobot. Bioinspiration \&s Biomimetics, vol. 6 , no.3, pp.036009, 2011. vii 19

[21] K. Meng, W. Zhang, W. Chen, H. Li, P. Chi, C. Zou, X. Wu, F. Cui, W. LIU, AND J. CHEN. The design and micromachining of an electromagnetic MEMS flapping-wing micro air vehicle. Microsystem technologies, vol.18, no.1, pp.127-136, 2012. vii 2023

[22] J. A. Roll, B. Cheng, and X. Deng. An Electromagnetic Actuator for High-Frequency Flapping-Wing Microair Vehicles. IEEE Transactions on Robotics, 31(2):400-414, 2015.

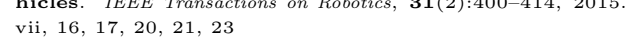

[23] J. Roll, B. Cheng, X. Deng, et AL. Design, fabrication, and experiments of an electromagnetic actuator for flapping wing micro air vehicles. In 2013 IEEE International Conference on Robotics and Automation (ICRA), pp.809-815, 2013. vii $2 0 \longdiv { 2 1 } 2 3$

[24] B. Cheng. Bio-inspired Aerial Robots. http://www.mne.psu.edu/bo/research.html, 2016. vii 21

[25] G.K. Lau, H.T. Lim, J.Y. Teo, and Y.W. Chin. Lightweight mechanical amplifiers for rolled dielectric elastomer actuators and their integration with bio-inspired wing flappers. Smart Materials and Structures, vol.23, no.2, pp.025021, 2014. vii $16 \quad 2223$

[26] J.J. Videler. Avian flight. Oxford University Press, 2006. vii 27

[27] G.K. Taylor, A.C. Carruthers, T.Y. Hubel, and S.M. Walker. Wing morphing in insects, birds and bats: mechanism and function. Morphing Aerospace vehicles and structures,pp.11-40, 2012. vii 2728,80

[28] D. Mueller, J.W. Gerdes, AND S.K. Gupta. Incorporation of passive wing folding in flapping wing miniature air vehicles. In ASME International Design Engineering Technical Conferences and Computers and Information in Engineering Conference, pp.797-805, 2009. vii 293237

[29] D. Billingsley, G. Slipher, J. Grauer, and J. Hubbard. Testing of a passively morphing ornithopter wing, vol.1828. AIAA Paper, 2009. vii $30 \quad 3137$ 
[30] Y. Tummala, A. Wissa, M. Frecker, and J.E. Hubbard. Design of a passively morphing ornithopter wing using a novel compliant spine. In ASME Conference on Smart Materials, Adaptive Structures and Intelligent Systems, pp.703-713, 2010. viii 3137

[31] A. Wissa, N. Guerreiro, J. Grauer, J.E. Hubbard JR, C. AltenbuchNer, Y. Tummala, M. Frecker, AND R. Roberts. Flight testing of novel compliant spines for passive wing morphing on ornithopters. In $54 T H$ AIAA/ASME/ASCE/AHS/ASC Conference Structures, Structural Dynamics, and Materials, 2013. viii 3137

[32] A. Wissa, J. Calogero, N. Wereley, J.E. Hubbard JR, and M. Frecker. Analytical model and stability analysis of the leading edge spar of a passively morphing ornithopter wing, vol.10, no.6, pp.065003. Bioinspiration \& biomimetics, 2015 viii 3137

[33] A.K. Stowers and D. LentinK. Folding in and out: passive morphing in flapping wings, vol.10, no.2, pp.025001. Bioinspiration \& biomimetics, 2015. viii $27,28,32$

[34] H. Tanaka, H. Okada, Y. Shimasue, and H. Liu. Flexible flapping wings with self-organized microwrinkles. Bioinspiration 8 biomimetics, vol.10,no.4,pp.046005, 2015. viii 33

[35] G. Bunget. BATMAV-A Bio-Inspired Micro-Aerial Vehicle for Flapping Flight. 2010. viii 343537

[36] S. Yang and S. Seelecke. FE analysis of SMA-based bioinspired bone-joint system. Smart Materials and Structures, vol.18, no.10, pp.104020, 2009. viii 343537

[37] Bunget G. BatMAV. https://sites.google.com/site/gheorghebunget/rese 2010. viii 35

[38] J.W. Bahlman, S.M. Swartz, And K.S. Breuer. Design and characterization of a multi-articulated robotic bat wing Bioinspiration Es biomimetics, vol.8, no.1, pp.016009, 2013. viii $2 8 \longdiv { 3 5 } 3 6,80,90$

[39] J.W. Bahlman, S.M. Swartz, and K.S. Breuer. How wing kinematics affect power requirements and aerodynamic force production in a robotic bat wing. Bioinspiration \&s biomimetics, vol.9, no.2, pp.025008, 2014. viii 3536

[40] J. Colorado, A. Barrientos, C. Rossi, Balhman J., and K.S. Breuer. Biomechanics of smart wings in a bat robot: morphing wings using SMA actuators. Bioinspiration \& biomimetics,

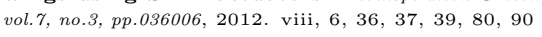

[41] R.D. Kornbluh, R. Pelrine, Q. Pei, R. Heydt, S. Stanford, S. Oh, and J. ECKERLE. Electroelastomers: applications of dielectric elastomer transducers for actuation, generation, and elastomer transducers for actuation, generation, and
smart structures. In SPIE's 9th Annual International Symposmart structures. In SPIE's 9th Annual International Sympo-
sium on Smart Structures and Materials, pp.254-270, 2002. खi 17 21

[42] T.A. Ward, M. Rezadad, C.J. Fearday, and R. Viyapuri. A Review of Biomimetic Air Vehicle Research: 1984-2014 In ternational Journal of Micro Air Vehicles, vol.7, no.3, pp.375-394, 2015. 1

[43] Z. Khan, K. Steelman, and S. Agrawal. Development of insect thorax based flapping mechanism. In IEEE International Conference on Robotics and Automation, pp.3651-3656, 2009. 1

[44] M. Ryan AND H.J. Su. Classification of flapping wing mechanisms for micro air vehicles. In International Design Engineering Technical Conferences and Computers and Information in Engineering Conference, pp.105-115, 2012. 1

[45] M. Keennon, K. Kuingebiel, H. Won, and A. Andriukov. Development of the nano hummingbird: A tailless flapping wing micro air vehicle. In AIAA aerospace sciences meeting,pp.1-24, 2012. 2
[46] J. JeOn, H. Сно, Y. H. KIM, J.H. LeE, S.J. Shis, C. KIM , AND S. AND отнERS KIM. Design and Analysis of the Link Mechanism in the Flapping Wing MAV Using Flexible Multi-Body Dynamic Analysis. In 24th AIAA/AHS Adaptive Structures Conference, page 0819, 2016. 2

[47] T.A. Nguyen, H.V. Phan, T.K.L. Au, And H.C. Park. Experimental study on thrust and power of flapping-wing system based on rack-pinion mechanism. Bioinspiration \& Biomimetics, vol.11, no.4,pp.046001, 2016. 2

[48] C.H. LiU AND C.K. Chen. Kinematic Analysis of a Flappingwing Micro-aerial-vehicle with Watt Straight-line Linkage. Journal of Applied Science and Engineering, vol.18, no.4, pp.355-362, 2015. 2

[49] L.L. HowelL. Compliant mechanisms. John Wiley \& Sons, 2001. 2214861

[50] R.J. Wood, B. Finio, M. Karpelson, K. Ma, N.O. Perez-Arancibia, P. Sreetharan, H. Tanaka, and J.P. Whitney. Progress on" pico" air vehicles. International Journal of Robotics Research, pp.0278364912455073, 2012. 288

[51] J.R. Bronson, J.S. Pulskamp, R.G. Polcawich, C.M. Kroninger, and E.D. Wetzel. PZT MEMS actuated flapping wings for insect-inspired robotics. In 22nd IEEE International Conference on Micro Electro Mechanical Systems (MEMS), pp.1047-1050, 2009. 288

[52] X.Q. Bao, T. Dargent, S. Grondel, J.B. Paquet, and E. Cattan. Improved micromachining of all SU-8 $3 \mathrm{D}$ structures for a biologically-inspired flying robot. Microelectronic Engineering, vol.88, no.8, pp.2218-2224, 2011. 288

[53] H.E. TAHA, M.R. HA.J, AND A.H. NAYFeh. Flight dynamics and control of flapping-wing MAVs: a review. Nonlinear Dymayamics, vol.70, no.2, pp.907-939, 2012. 2

[54] G. Cai, J. Dias, and L. Seneviratne. A survey of small-scale unmanned aerial vehicles: Recent advances and future development trends, vol.2,no.2,pp.175-199. Unmanned Systems, 2014. 2890

[55] X. Deng, L. Schenato, W.C. Wu, and S.S. Sastry. Flapping flight for biomimetic robotic insects: part I-system modeling. IEEE Transactions on Robotics, vol.22,no.4,pp.776-788, 2006. 26390

[56] X. Deng, L. Schenato, and S.S. Sastry. Flapping flight for biomimetic robotic insects: part II-flight control design. IEEE Transactions on Robotics, vol.22,no.4,pp.789-803, 2006. 2490

[57] J.A Grauer and J.E. Hubbard. Multibody model of an ornithopter. Journal of guidance, control, and dynamics, vol.32,no.5,pp.1675-1679, 2009. 36490

[58] J. Colorado, C. Rossi, C. Zhang, and A. Barrientos. Towards efficient flight: insights on proper morphing-wing modulation in a bat-like robot. Advanced Robotics, vol.29, no.24, pp.1599-1610, 2015. 3636373980,90

[59] C. De Wagter, A. Koopmans, G.C.H.E. de Croon, B.D.W. Remes, and R. RUIJINK. Autonomous wind tunnel free-flight of a flapping wing MAV. Advances in Aerospace Guidance, Navigation and Control,pp.603-621, 2013. 362

[60] S.S Baek, F.L. Garcia Bermudez, and R.S. Fearing. Flight control for target seeking by $\mathbf{1 3}$ gram ornithopter. In IEEE/RSJ International Conference on Intelligent Robots and Systems (IROS), pp.2674-2681. IEEE, 2011. 362

[61] P.J. Duhamel, N.O. Pérez-Arancibia, G.L. Barrows, and R.J. Wood. Altitude feedback control of a flapping-wing microrobot using an on-board biologically inspired optical flow sensor. In 2012 IEEE International Conference on Robotics and Automation (ICRA), pp.4228-4235. IEEE, 2012. 362

[62] P. Chirarattananon, K.Y. Ma, and R.J. Wood. Adaptive control for takeoff, hovering, and landing of a robotic fly. In 2013 IEEE/RSJ International Conference on Intelligent Robots and Systems (IROS), pp.3808-3815. IEEE, 2013. 3 26 62 
[63] N.O. Pérez-Arancibia, P.J. Duhamel, K.y. Ma, and R.J. Wood. Model-free control of a flapping-wing flying microrobot. In 2013 16th International Conference on Advanced Robotics (ICAR), pp.1-8. IEEE, 2013. 362

[64] A. Lampton, A. Niksch, and J. Valasek. Reinforcement learning of morphing airfoils with aerodynamic and strucing of morphing airfoils with aerodynamic and struc-
tural effects. Journal of Aerospace Computing, Information, and

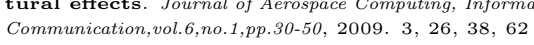

[65] A. Lampton, A. NiKsch, and J. Valasek. Reinforcement learning of a morphing airfoil-policy and discrete learning analysis. Journal of Aerospace Computing, Information, and Communication, vol.7,no.8,pp.241-260, 2010. 3263862

[66] S. Barbarino, O. Bilgen, R.M. Aja.j, M.I. Friswell, and D.J. Inman. A review of morphing aircraft. Journal of Intelligent Material Systems and Structures, vol.22,no.9,pp.823-877, 2011. 428

[67] William W Gilbert. Mission adaptive wing system for tactical aircraft. Journal of Aircraft, 18(7):597-602, 1981. 4

[68] Wlezien RW, Horner GC, McGowan AR, Padula SL, Scott Ma, SiLcox RJ, and Simpson JO. The Aircraft Morphing Program 1998. 4

[69] Adam M Wickenheiser and Ephrahim Garcia. Optimal trajectory control of morphing aircraft in perching maneuvers. Morphing Aerospace Vehicles and Structures, pages 177203, 2012. 4

[70] J.C. GomeZ And E. GarCiA. Morphing unmanned aerial vehicles. Smart Materials and Structures, vol.20, no.10, pp.103001, 2011. 田 28

[71] G. ABATE AND W. SHYY. Bio-Inspiration of Morphing for Micro Air Vehicles. Morphing Aerospace Vehicles and Structures,pp.41-53, 2011. 46280

[72] A. Niksch, J. Valasek, T.W. Strganac, and L.A. Carlson. Morphing aircraft dynamical model: Longitudinal shape changes. In AIAA Atmospheric Flight Mechanics Conference and Exhibit, pp.6567, 2008. 4

[73] J. Niksch, A.and Valasek, T.W. Strganac, and L.A. Carlson. Six degree of freedom dynamical model of a morphing aircraft. In Proc. of AIAA Atmospheric Flight Mechanics Conference, 2009. 4

[74] M.L. Verstraete, S. Preidikman, B.A. Roccia, and D. T. Мook. A Numerical Model to Study the Nonlinear and Unsteady Aerodynamics of Bioinspired Morphing-Wing steady Aerodynamics of Bioinspired Morphing-Wing
Concepts. International Journal of Micro Air Vehicles, vol.7, Concepts. International
no.3, pp.327-346, 2015. 4

[75] M.V. Donadon and L. Iannucci. A numerical study on smart material selection for flapped and twisted morphing wing configurations. Journal of Aerospace Technology and Management, vol.6, no.3, pp.281-290, 2014. 433

[76] T. WeIs-Fogh. Elasticity and wing movements in insects. Proceedings XII Int. Congress of Entomology, pp.186-188, 1965. 10

[77] M.H. Dickinson and J.R.B. Lighton. Muscle efficiency and elastic storage in the flight motor of Drosophila. Science, vol.268,no.5207,pp.87-90, 1995. 10

[78] R.F. Chapman. The insects: structure and function. Cambridge university press, 1998. 10

[79] R.J. Wоотton. Springy shells, pliant plates and minimal motors: Abstracting the insect thorax to drive a micro-air vehicle. In Flying insects and robots, pp.207-217. Springer, 2009. 10

[80] P.J. Gullan and P.S. Cranston. The insects: an outline of entomol ogy. John Wiley \& Sons, 2009. 10

[81] J.L. NATION. Insect physiology and biochemistry. CRC press, 2008. 10
[82] Z.A. Khan and S.K. Agrawal. Optimal hovering kinematics of flapping wings for micro air vehicles. AIAA journal, vol.49, no.2, pp.257-268, 2011. 11

[83] S.K. Park, J.H.and Agrawal. Dynamic effects of Asymmetric In-Phase Flapping (AIF) on forward flight. In IEEE International Conference on Robotics and Automation (ICRA), pp.3550-3555, 2014. 11

[84] D. Campolo, M. Azhar, G.K. Lau, and M. Sitti. Can DC motors directly drive flapping wings at high frequency and large wing strokes? IEEE/ASME Transactions on Mechatronics, vol.19, no.1, pp.109-120, 2014. 12

[85] D. Colmenares, R. Kania, W. Zhang, and M. Sitti. Compliant wing design for a flapping wing micro air vehicle. In LEEE/RSJ International Conference on Intelligent Robots and Systems (IROS), pp.32-39, 2015. 13

[86] Lindsey Hines, David Colmenares, and Metin Sitti. Platform design and tethered flight of a motor-driven flappingwing system. In IEEE International Conference on Robotics and Automation (ICRA), pp.5838-5845, 2015. 13

[87] T. Tantanawat and S. Kota. Design of compliant mechanisms for minimizing input power in dynamic applications. Journal of Mechanical Design, vol.129, no.10, pp.1064-1075, 2007. 1341

[88] Y.W. Chin and G.K. LAU. Clicking compliant mechanism for flapping-wing micro aerial vehicle. In IEEE/RSJ International Conference on Intelligent Robots and Systems (IROS), pp.126-131, 2012. 1441

[89] M. SiтTI. Piezoelectrically actuated four-bar mechanism with two flexible links for micromechanical flying insect thorax. IEEE/ASME Transactions on Mechatronics, vol.8,

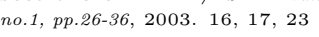

[90] M. YoICHI. Applications of piezoelectric actuator. NEC Technical Journal, vol.1,no.5,pp.82-86, 2006. 16

[91] T. Dargent, X.Q. Bao, S. Grondel, G. Le Brun, J.B. Paquet, C. Soyer, AND E. CATTAN. Micromachining of an SU-8 flapping-wing flying micro-electro-mechanical system. Journal of Micromechanics and Microengineering, vol.19, n0.8, pp. 085028, 2009. 16 20

[92] P. Zdunich, D. Bilyk, M. MacMaster, D. Loewen, J. DeLaurier, R. KoRNBluH, T. Low, S. StanFord, AND D. Holeman. Development and testing of the mentor flapping-wing micro air vehicle. Journal of Aircraft, vol.44, no.5, pp.1701-1711, 2007. 16]21.

[93] M. Karpelson, G.Y. Wei, and R.J. Wood. A review of actuation and power electronics options for flapping-wing robotic insects. In IEEE International Conference on Robotics and Automation (ICRA), pp.779-786, 2008. 17,90

[94] K. Mateti, R.A. Byrne-Dugan, S.A. Tadigadapa, and C.D. Rahn. SUEX flapping wing mechanisms for Pico Air Vehicles. In ASME Conference on Smart Materials, Adaptive Structures and Intelligent Systems, pp.583-590, 2012. 17]

[95] V. Arabagi, L. Hines, and M. Sitti. A simulation and design tool for a passive rotation flapping wing mechanism. IEEE/ASME Transactions on Mechatronics, vol.18, no.2, pp.787$798,2013.19$

[96] P.S. SReetharan and R.J Wood. Passive torque regulation in an underactuated flapping wing robotic insect. $A u-$ tonomous robots, vol.31,no.2-3,pp.225-234, 2011. 19

[97] M. LoK, D. BRooks, R. Wood, AND G.Y. Wei. Design and analysis of an integrated driver for piezoelectric actuators. In IEEE Energy Conversion Congress and Exposition (ECCE), pp. 26842691, 2013. 19

[98] M. LoK, X. Zhang, E.F. Helbling, R. Wood, D. Brooks, and G.Y. Wei. A power electronics unit to drive piezoelectric actuators for flying microrobots. In IEEE Custom Integrated Circuits Conference (CICC), pp.1-4, 2015. 19 
[99] B. Cheng, J.A. Roll, and X. Deng. Modeling and optimization of an electromagnetic actuator for flapping wing micro air vehicle. In 2013 IEEE International Conference on Robotics and Automation (ICRA), pp.4035-4041. IEEE, 2013.20 23

[100] J. D.W. Madden, N.A. Vandesteeg, P.A. Anguetil, P.ga Madden, A. TAKshi, R.Z. Pytel, S.R. Lafontaine, P.A. Wieringa, and I.W A. TAKSHI, R.Z. Artificial muscle technology: physical princiHUNTER. Artificial muscle technology: physical princi-
ples and naval prospects. IEEE Journal of oceanic engineering, vol.29, no.3, pp.706-728, 2004. 21

[101] K.J. Kim AND S. TADOKORO. Electroactive polymers for robotic applications. Artificial Muscles and Sensors (291 p.), Springer: London, United Kingdom, 2007. 21

[102] R. Pelrine, R.D. Kornbluh, S. Pei, Q.And Stanford, S. OH, J. Eckerle, R.J. Full, and K. Rosenthal, M.A .and Meijer. Dielectric elastomer artificial muscle actuators: toward biomimetic motion. In SPIE's 9th Annual International Symposium on Smart Structures and Materials, pp.126-137, 2002. 21

[103] X. YAN, M. QI, AND L. LiN. Self-lifting artificial insect wings via electrostatic flapping actuators. In 28th IEEE Inter-
national Conference on Micro Electro Mechanical Systems (MEMS), pp.22-25, 2015. 22

[104] Z. LIU, X. YAN, M. QI, AND L. LIN. Electrostatic flapping wings with pivot-spar brackets for high lift force. In 29th International Conference on Micro Electro Mechanical Systems (MEMS), pp.1133-1136, 2016. 22

[105] M.W. Oppenheimer, D.B. Doman, and D. Sigthorsson. Dynamics and control of a minimally actuated biomimetic vehicle: Part ii-control. In Proceedings of the AIAA Guidance, Navigation, and Control Conference,pp.1-23, 2009. 23,24

[106] D.B. Doman, M.W. Oppenheimer, and D. Sigthorsson. Dynamics and control of a minimally actuated biomimetic vehicle: Part i-aerodynamic model. In Proceedings of the AIAA Guidance, Navigation, and Control Conference, pp.10-13, 2009. 23

[107] D.B. Doman, M.W. Oppenheimer, and D.O. Sigthorsson. Wingbeat shape modulation for flapping-wing micro-air-vehicle control during hover. Journal of guidance, control, and dynamics, vol.33, no.3, pp.724-739, 2010. 24,25

[108] M.W. Oppenheimer, D.B. Doman, and D.o. Sigthorsson. Dynamics and control of a biomimetic vehicle using biased wingbeat forcing functions: Part I-Aerodynamic model. In Proceedings of the 48th AIAA Aerospace Sciences Meeting Including the New Horizons Forum and Exposition, pp.1-34, 2010. 24

[109] D.B. Doman, M.W. Oppenheimer, and D.O. Sigthorsson. Dynamics and control of a biomimetic vehicle using biased wingbeat forcing functions: Part ii: Controller. AIAA Washington, vol.1024, 2010. 24

[110] X. Deng, L. Schenato, and S. Sastry. Hovering flight control of a micromechanical flying insect. In Proceedings of the 40 th IEEE Conference on Decision and Control, vol.1, pp.235-240. IEEE, 2001. 24

[111] B. Cheng and X. Deng. Near-hover dynamics and attitude stabilization of an insect model. In Proc. American Control Conf, pages 39-44, June 2010. 24

[112] S.P. SAne And M.H. Dickinson. The aerodynamic effects of wing rotation and a revised quasi-steady model of flapping flight. Journal of experimental biology, vol.205, no.8, pp.1087-1096, 2002. 24

[113] Y. XIONG AND M. SUn. Stabilization control of a bumblebee in hovering and forward flight. Acta Mechanica Sinica, vol.25, no.1, pp.13-21, 2009. 25

[114] M. SUn AND Y. XIONG. Dynamic flight stability of a hovering bumblebee. Journal of Experimental Biology, vol.208, no.3 pp.447-459, 2005. 25
[115] Y XIONG AND M. SUN Dynamic flight stability of a bumblebee in forward flight. Acta Mechanica Sinica, vol.24, no.1, pp.25-36, 2008. 25

[116] J. Geder, R. Ramamurti, W. Sandberg, and A. Flynn. Modeling and control design for a flapping-wing nano air vehicle. In Proceedings of the AIAA guidance, navigation, and control conference. pp.1-15, 2010. 25

[117] A. SERRANI. Robust hovering control of a single-dof flapping mav. In Proceedings of the 2010 American Control Conference, vol. 30, pp.1302-1307. 25

[118] A. Serrani, B.E. Keller, M.A. Bolender, and D.B. Doman. Robust control of a 3-dof flapping wing micro air vehicle. In AIAA Guidance, Navigation and Control Conference, vol.7709, pp.2010, 2010. 25

[119] M.W. Oppenheimer, D.B. Doman, and D.O. Sigthorsson. Dynamics and control of a biomimetic vehicle using biased wingbeat forcing functions. Journal of guidance, control, and dynamics, vol.34, no.1, pp.204-217, 2011. 25

[120] M.A. BoleNDER. Rigid multi-body equations-of-motion for flapping wing MAVs using Kanes equations. In AIAA Guidance, Navigation, and Control Conference, no.2009-6158, 2009. 25

[121] H. Rifal, N. MARChand, AND G. Poulin, Bounded control of a flapping wing micro drone in three dimensions. In
IEEE International Conference on Robotics and Automation (ICRA), pp.164-169. IEEE, 2008. 25

[122] I. Faruque and J.S. Humbert. Dipteran insect flight dynamics. Part 1 Longitudinal motion about hover. Journal of theoretical biology, vol.264, no.2, pp.538-552, 2010. 26

[123] J.S. Humbert and I.A. Faruque. Analysis of insect-inspired wingstroke kinematic perturbations for longitudinal
control. Journal of Guidance, Control, and Dynamics, vol.34, no.2, pp.618-623, 2011. 26

[124] H. Duan and Q. Li. Dynamic model and attitude control of flapping wing micro aerial vehicle. In 2009 IEEE International Conference on Robotics and Biomimetics (ROBIO), pp.451456. IEEE, 2009. 266364

[125] H. DUAN AND D. Gu. Sliding mode adaptive control for flying robot based on recurrent CMAC algorithm. In IEEE International Conference on Mechatronics and Automation, pp.440-445. IEEE, 2011. 2664

[126] Q. Guo, M. Hu, R. Wei, J. Xu, And H. Song. Hovering control based on fuzzy neural networks for biomimetic fying robotic. In International Conference on Information and Automation (ICIA), pp.504-508. IEEE, 2008. 2638

[127] M. Hu, R. WeI, Z. Shi, AND L. Zou. Controllability issues for insect like flapping wing micro-air vehicle. In 7 th World Congress on Intelligent Control and Automation (WCICA), pp.63656368,. IEEE, 2008. 26

[128] S.J. ChUng AND M. DoRothy. Neurobiologically inspired control of engineered flapping flight. Journal of guidance, control, and dynamics, vol.33, no.2, pp.440-453, 2010. 27

[129] S.K. BoddhU AND J.C. GaLlagher. Evolving non-autonomous neuromorphic flight control for a flapping-wing mechanical insect. In IEEE Workshop on Evolvable and Adaptive Hardware (WEAH), pp.9-16. IEEE, 2009. 27

[130] D. Lentink, U.K. Müller, E.J. Stamhuis, R. De Kat, W. Van Gestel, L.L.M. Veldhuis, P. Henningsson, A. Hedenström, J.J. Videler, And J.L. VAN LeEuwen. How swifts control their glide performance with morphing wings. Nature, vol.446, no.7139, pp.1082-1085, 2007. 27

[131] Anna C Carruthers, adrian lR thomas, and Graham K Taylor. Automatic aeroelastic devices in the wings of a steppe eagle Aquila nipalensis. Journal of Experimental Biology, vol.210, no.23, pp.4136-4149, 2007. 27 
[132] C.J. Pennycuick. The Feathered Wings Of Birds. Theoretical Ecology Series, vol.5, pp.105-134, 2008. 27

[133] C.J. Pennycuick. The Membrane Wings of Bats and Pterosaurs. Theoretical Ecology Series, vol.5, pp.135-160, 2008. 27

[134] J. Tian, X.and Iriarte-Diaz, K. Middleton, R. Galvao, E. Israeli, A. Roemer, A. Sullivan, A. Song, S. Swartz, And K. Breuer. Direct measurements of the kinematics and dynamics of bat flight. Bioinspiration \&s Biomimetics,vol.1,no.4,pp.S10, 2006. 28

[135] J. Iriarte-Díaz, D.K Riskin, D.J. Wiluis, K.S. Breuer, and S.M. Swartz. Whole-body kinematics of a fruit bat reveal the influWhole-body kinematics of a fruit bat reveal the influ-
ence of wing inertia on body accelerations. Journal of experimental biology,vol.214,no.9,pp.1546-1553, 2011. 28

[136] T.Y. Hubel, N.I. Hristov, S.M. Swartz, and K.S. Breuer. Changes in kinematics and aerodynamics over a range of speeds in Tadarida brasiliensis, the Brazilian free-tailed bat. Journal of The Royal Society Interface, rsif20110838, 2012. 28

[137] S.M. Swartz, J. Iriarte-Diaz, D.K. Riskin, A. Song, X. Tian, D.J. WILLIS, AND K.S. BREUER. Wing structure and the aerodynamic basis of flight in bats. AIAA journal, vol.1, 2007. 28

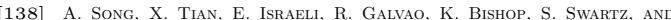
K. BREUER. Aeromechanics of membrane wings with imK. BREUER. Aeromechanics of membrane wings with im-
plications for animal flight. AIAA journal, vol.46, no.8, pp.2096-2106, 2008. 28

[139] D.T. Grant, M. Abdulrahim, And R. Lind. Flight dynamics of a morphing aircraft utilizing independent multiple-joint wing sweep. In AIAA Atmospheric Flight Mechanics Conference and Exhibit, pp.1111-1125, 2006. 28

[140] D.T. Grant, M. Abdulrahim, and R. Lind. Design and analysis of biomimetic joints for morphing of micro air vehicles. Bioinspiration \&3 biomimetics, vol.5, no.4, pp.045007, 2010. 28

[141] FESTO Company. Aerodynamic lightweight design with active torsion. https://www.festo.com, 2011. 34 37

[142] W. Send, M. Fischer, K. Jebens, R. Mugrauer, A. Nagarathinam, and F. Scharstein. Artificial hinged-wing bird with active torsion and partially linear kinematics. In 28th Congress of the International Council of the Aeronautical Sciences,pp.23-28, 2012. 3437

[143] R. Callahan and E. Garcia. Bio-inspired bat wing design and fabrication. Cornell University, Ithaca, NY, vol.14850, pp.10, 2010. 35

[144] E.A. Leylek, J.E. Manzo, and E. Garcia. Bat-inspired wing aerodynamics and optimization. Journal of Aircraft, vol.47, no.1, pp.323-328, 2010. 35

[145] J. Colorado, A. Barrientos, C. Rossi, and C. Parra. Inertial attitude control of a bat-like morphing-wing air vehicle,vol.8,no.1,pp.016001,. Bioinspiration \& biomimetics, 2012. 36,80

[146] G. Bunget and S. Seelecke. BATMAV: a biologically inspired micro air vehicle for flapping flight: kinematic spired micro air vehicle for flapping flight: kinematic tures and Materials 83 Nondestructive Evaluation and Health Monitures and Materials \& Nondestructive
toring, pp.69282F-69282F, 2008. 37

[147] J.P. Khatait, S. Mukher.jee, and B. Seth. Compliant design for flapping mechanism: A minimum torque approach. Mechanism and Machine Theory, vol.41, no.1, pp.3-16, 2006. 38 618

[148] B. Cheng And X. Deng. A neural adaptive controller in flapping flight. Journal of Robotics and Mechatronics, vol.24, no.4 pp.602-611, 2012. 38
[149] G.A Pratt and M.M Williamson. Elastic actuator for precise force control, 1997. US Patent 5,650,704. 40

[150] R.J. Wood. Liftoff of a 60mg flapping-wing MAV. In $I E E E / R S J$ International Conference on Intelligent Robots and Systems (IROS), pp.1889-1894, 2007. 41

[151] A. Cox, D. Monopoli, D. Cveticanin, M. Goldfarb, and E. Garcia. The development of elastodynamic components for piezoelectrically actuated flapping micro-air vehicles. Journal of Intelligent Material Systems and Structures, vol.13, no.9, pp.611-615, 2002. 41

[152] L.L Howell and A. Midha. A method for the design of compliant mechanisms with small-length flexural pivots. Journal of Mechanical Design, vol.116, no.1, pp.280-290, 1994. 42

[153] L.L Howell, A. MidHA, AND T.W. Norton. Evaluation of equivalent spring stiffness for use in a pseudo-rigid-body model of large-deflection compliant mechanisms. Journal of Mechanical Design, vol.118, no.1, pp.126-131, 1996. 42

[154] Y.Q. Yu, L.L. Howeld, C. Lusk, Y. Yue, and M.G. He. Dynamic modeling of compliant mechanisms based on the pseudo-rigid-body model. Journal of Mechanical Design, vol.127, no.4, pp.760-765, 2005. 4254

[155] C. Zhang, C. Rossi, W. He, and J. Colorado. Virtual-workbased Optimization Design on Compliant Transmission Mechanism for Flapping-wing aerial Vehicles. In International Conference on Manipulation, Automation and Robotics at Small Scales (MARSS), 2016.

[156] CP ElLington. The aerodynamics of hovering insect flight. I. The quasi-steady analysis. Philosophical Transactions of the Royal Society B: Biological Sciences, vol.305, no.1122, pp.1-15, 1984. 50 5161

[157] Y. WANG AND Z. CAI. A hybrid multi-swarm particle swarm optimization to solve constrained optimization problems. Frontiers of Computer Science in China, vol.3, no.1, pp.3852, 2009. 4561

[158] Z.A. Khan And S.K. Agrawal. Design of flapping mechanisms based on transverse bending phenomena in insects. In IEEE International Conference on Robotics and Automation (ICRA), pp.2323-2328, 2006. 51

[159] R.A. Aliev and B.G. Guirimov. Type-2 Fuzzy Neural Networks and Their Applications. Springer, 2014. 6263

[160] Y.X. LIAO, J.H. SHE, AND M. WU. Integrated hybrid-PSO and fuzzy-NN decoupling control for temperature of reheating furnace. IEEE transactions on industrial electronics, vol.56, no.7, pp.2704-2714, 2009. 6269

[161] I.H. Li, W.Y. Wang, S.F. Su, and Y.S. LeE. A merged fuzzy neural network and its applications in battery state-ofcharge estimation. IEEE Transactions on Energy Conversion, charge estimation. IEEE Tran.
vol.22, no.3, pp.697-708, 2007. 62

[162] C.T. Lin, C.M. Yeh, S.F. Liang, J.F. Chung, And N. Kumar. Supportvector-based fuzzy neural network for pattern classification. IEEE Transactions on Fuzzy Systems, vol.14, no.1, pp.3141, 2006. 62

[163] K.Y. Chan and T.S. Dillon. On-road sensor configuration design for traffic flow prediction using fuzzy neural
networks and taguchi method. IEEE Transactions on Innetworks and taguchi method. IEEE Transactions on In-
strumentation and Measurement, vol.62, no.1, pp.50-59, 2013. 62

[164] H. Gomi and M. Kawato. Neural network control for a closed-loop system using feedback-error-learning. Neural Networks, vol.6, no.7, pp.933-946, 1993. 6365

[165] M. A. Khanesar, E. Kayacan, M. Reyhanoglu, and O. Kaynak. Feedback error learning control of magnetic satellites using type-2 fuzzy neural networks with elliptic membership functions. IEEE transactions on cybernetics, vol.45, no.4, pp.858-868, 2015. 63 74 87 107 
[166] Z. KHAN. Modeling, optimal kinematics, and flight control of bioinspired flapping wing micro air vehicles. University of Delaware, 2009. 63

[167] M. LASEK AND K. SiBILSKI. Modelling and simulation of flapping wing control for a micromechanical flying insect (entomopter). In Modelling and Simulation Technologies Conference and Exhibit, vol.4973, no.2002. AIAA, 2002. 64.

[168] J. Grauer, E. Ulrich, J.E. Hubbard, D. Pines, and J.S. Humbert. Testing and system identification of an ornithopter in longitudinal flight. Journal of Aircraft, vol.48, no.2, pp.660$667,2011.64$

[169] T. Takagi and M. Sugeno. Fuzzy identification of systems and its applications to modeling and control. IEEE transactions on systems, man, and cybernetics, no.1, pp.116-132, 1985. 68

[170] M. SUGENo AND G.T. KANG. Structure identification of fuzzy model. Fuzzy sets and systems, vol.28, no.1,pp.15-33, 1988. 68

[171] J.M. MENDEL. Uncertain rule-based fuzzy logic systems: introduction and new directions. 2001. 68

[172] M.B. Begian, W.W. MeleK, And J.M. Mendel. Stability analysis of type-2 fuzzy systems. In IEEE International Conference on Fuzzy Systems, pp.947-953. IEEE, 2008. 68

[173] E. Kayacan, E. KayaCAN, AND M.A. Khanesar. Identification of nonlinear dynamic systems using type-2 fuzzy neural networks $A$ novel learning algorithm and a comparanetworksA novel learning algorithm and a compara-
tive study. IEEE Transactions on Industrial Electronics, vol.62, tive study. IEEE Transaction.
no.3,pp.1716-1724, 2015. 69

[174] E. Kayacan, E. Kayacan, H. Ramon, O. Kaynak, and W. Saeys. Towards agrobots: Trajectory control of an autonomous tractor using type-2 fuzzy logic controllers. $I E E E / A S M E$ Transactions on Mechatronics, vol.20, no.11, pp.287-298, 2015. 69 71

[175] C.H. Lee, J.L. Hong, Y.C. Lin, and W.Y. Lai. Type-2 fuzzy neural network systems and learning. International Journal of Computational Cognition, vol.1, no.4, pp.79-90, 2003. 69

[176] S.P. Panigrahi, S.K. NAYAK, And S.K. Padhy. A genetic-based neuro-fuzzy controller for blind equalization of timevarying channels. International Journal of Adaptive Control and Signal Processing, vol.22, no.7, pp.705-716, 2008. 69

[177] G.S. Kiм, I.S. Ahn, AND S.K. ОH. The design of optimized type-2 fuzzy neural networks and its application. The type-2 fuzzy neural networks and its application. The
Transactions of The Korean Institute of Electrical Engineers, vol.58, Transactions of The Korean In
no.8, pp.1615-1623, 2009. 69

[178] C.F. Juang and C.H. Hsu. Reinforcement interval type2 fuzzy controller design by online rule generation and Q-value-aided ant colony optimization. IEEE Transactions on Systems, Man, and Cybernetics, Part B (Cybernetics), vol.39, no.6, pp.1528-1542, 2009. 69

[179] S. YU, X. YU, AND Z. MAN. A fuzzy neural network approximator with fast terminal sliding mode and its applications. In Proceedings of the 9th International Conference on Neural Information Processing (ICONIP), vol.3, pp.1257-1261. IEEE, 2002. 71

[180] Gl Cascella, F Cupertino, aV Topalov, O Kaynak, and V Giordano. Adaptive control of electric drives using sliding-mode learning neural networks. In Proceedings of the IEEE International Symposium on Industrial Electronics (ISIE), vol.1, pp.125$130,2005.71$

[181] M.O. EFE, O. KAYNAK, AND X. YU. Sliding mode control of a three degrees of freedom anthropoid robot by driving the controller parameters to an equivalent regime. Journal of Dynamic Systems, Measurement, and Control, vol.122, no.4, pp.632-640, 2000. 71
[182] E. KAYACAN, O. CIGDEM, AND O. KAYNAK, Sliding mode control approach for online learning as applied to type-2 fuzzy neural networks and its experimental evaluation. IEEE Transactions on Industrial Electronics, vol.59, no.9, pp.3510-3520, 2012. 71

[183] V.I. UtкIN. Sliding modes in control and optimization. Springer Science \& Business Media, 2013. 73

[184] J. Iriarte-Díaz, D.K. Riskin, D.J. Willis, K.S. Breuer, and S.M. SWARTZ. Whole-body kinematics of a fruit bat reveal the influence of wing inertia on body accelerations. Journal of Experimental Biology, vol. 214, no. 9, pp. 1546-1553, 2011. 80

[185] D.K. Riskin, J. Iriarte-Díaz, K.M. Middleton, K.S. Breuer, and S.M. SWARTZ. The effect of body size on the wing movements of pteropodid bats, with insights into thrust and lift production. Journal of Experimental Biology, vol. 213 (23) pp. production. Journal

[186] M. Thollesson and U.M. Norberg. Moments of inertia of bat wings and body. Journal of experimental Biology, vol.158, no.1, pp.19-35, 1991. 80

[187] L.L. HoweLl. Compliant Mechanisms, pages 457-463. Springer Netherlands, 2012. 87

[188] T.J. Teo, G. Yang, And I.M. Chen. Compliant Manipulators, pages 2229-2300. Springer London, London, 2015. 88

[189] D. Kern, J. Bauer, and W. Seemann. Control of compliant mechanisms with large deflections. In Advances in Mechanisms Design, pp.193-199. Springer, 2012. 88

[190] K. Fite and M. Goldfarb. Position control of a compliant mechanism based micromanipulator. In IEEE International Conference on Robotics and Automation (ICRA), vol.3, pp.2122-2127. IEEE, 1999. 88

[191] J. Speich and M. Goldfarb. A compliant-mechanism-based three degree-of-freedom manipulator for small-scale manipulation. Robotica, vol.18, no.1, pp.95-104, 2000. 88

[192] A. SChNeuwly. Charge ahead [ultracapacitor technology and applications]. Power Engineer, vol.19,no.1,pp.34-37, 2005. 89

[193] A. Perez-Rosado, H.A. Bruck, and S.K. Gupta. Integrating Solar Cells Into Flapping Wing Air Vehicles for Enhanced Flight Endurance. Journal of Mechanisms and hanced Flight Endurance. Jour
Robotics, vol.8,no.5, pp.051006, 2016. 89

[194] A. Wilhelm, B.W. Surgenor, and J.G. Pharoah. Evaluation of a micro fuel cell as applied to a mobile robot. In IEEE International Conference Mechatronics and Automation, vol.1, pp.3236,. IEEE, 2005. 89

[195] A. Evans, A. Bieberle-Hütter, J.L.M. Rupp, and L.J. Gauckler. Review on microfabricated micro-solid oxide fuel cell membranes. Journal of Power Sources, vol.194, no.1, pp.119-129, 2009. 89

[196] C.K. Chan, H. Peng, G. Liv, K. Mcllwrath, X.F. Zhang, R.A. Huggins, AND Y. CUI. High-performance lithium battery anodes using silicon nanowires. Nature nanotechnology,vol.3,no.1,pp.31-35, 2008. 89

[197] B. Kumar, J. Kumar, R. Leese, J.P. Fellner, S.J. Rodrigues, and K.M. Aвraham. A solid-state, rechargeable, long cycle life lithium-air battery. Journal of The Electrochemical Society, vol.157,no.1, pp.A50-54, 2010. 89

[198] M. Karpelson, G.Y. Wei, and R.J. Wood. Milligram-scale high-voltage power electronics for piezoelectric microrobots. In 2009 IEEE International Conference on Robotics and Automation (ICRA), pp.2217-2224. IEEE, 2009. 89

[199] K. Uchino, B. Koc, P. Laoratanakul, and A. Carazo. Piezoelectric transformers: New perspective. Ferroelectrics, vol.263, no.1,pp.91-100, 2001. 89 
[200] D. Campolo, M. SitTi, AND R.S. FFaring Efficient charge recovery method for driving piezoelectric actuators with quasi-square waves. IEEE transactions on ultrasonics, ferroelectrics, and frequency control, vol.50, no.3, pp.237-244, 2003. 89

[201] E. Steltz, M. Seeman, S. Avadhanula, and R.S. Fearing. Power electronics design choice for piezoelectric microelectronics design choice for piezoelectric micro-
robots. In 2006 IEEE/RSJ International Conference on Intelligent Robots and Systems, pp.1322-1328. IEEE, 2006. 89

[202] C. Chen, Y. Tang, A. Khaligh, and R.W. Newcomb. A low-power and high-gain converter for driving dielectric elastomer actuators. In 2013 Tur dighth A tomer actuators. In 2013 Thenty Eigh d AnMaAl IEEE Ap plied Power Eleter Conference and Exposition (APEC), pP. 2760. IEEE, 2013. 89
[203] C. Chen, Y. Tang, H. Wang, And Y. Wang. A Review of Fabrication Options and Power Electronics for FlappingWing Robotic Insects International Journal of Advanced Robotic Systems, vol.10,no.151, 2013. 90

[204] A. Ramezani, X. Shi, S.J. Chung, and S. Hutchinson. Bat Bot (B2), A Biologically Inspired Flying Machine. In Proceedings of the IEEE International Conference on Robotics and Automation (ICRA), 2016. 90

[205] A. Ramezani, X. Shi, S.J. Chung, and S. Hutchinson. Modeling and Nonlinear Flight Controller Synthesis of a BatInspired Micro Aerial Vehicle. 2016. 90,91

[206] J. Colorado, A. Barrientos, C. Rossi, and C. Parra. Inertial attitude control of a bat-like morphing-wing air vehicle. Bioinspiration Es biomimetics, vol.8, no.1, pp.016001, 2012. 91 


\section{Declaration}

I herewith declare that I have produced this thesis document without the prohibited assistance of third parties and without making use of aids other than those specified; notions taken over directly or indirectly from other sources have been identified as such.

The thesis work was conducted from 2012 to 2016 under the supervision of Prof. Claudio Rossi, $\mathrm{PhD}$.

Madrid, Spain 2016 
8

\section{Annexes}

\subsection{Proof of Theorem 1}

In this section, we will analyse the stability of the SMC theory-based learning algorithm for the IT2FNN using triangular MFs with uncertain width. At the beginning, these following variables are defined as

$$
\begin{aligned}
& \underline{A}_{1 i}=\frac{e-c_{1 i}}{\underline{d}_{1 i}}, \\
& \bar{A}_{1 i}=\frac{e-c_{1 i}}{\bar{d}_{1 i}} \\
& \underline{B}_{2 j}=\frac{\dot{e}-c_{2 j}}{\underline{d}_{2 j}} \\
& \bar{B}_{2 j}=\frac{\dot{e}-c_{2 j}}{\bar{c}_{2 j}} .
\end{aligned}
$$

Then, their corresponding derivatives with respect with time are

$$
\begin{aligned}
\dot{A}_{1 i} & =\frac{\dot{e}-\dot{c}_{1 i}}{\underline{d}_{1 i}}-\frac{\left(e-c_{1 i}\right) \dot{d}_{1 i}}{\underline{d}_{1 i}^{2}} \\
\dot{\bar{A}}_{1 i} & =\frac{\dot{e}-\dot{c}_{1 i}}{\bar{d}_{1 i}}-\frac{\left(e-c_{1 i}\right) \dot{\bar{d}}_{1 i}}{\bar{d}_{1 i}^{2}} \\
\underline{B}_{2 j} & =\frac{\ddot{e}-\dot{c}_{2 j}}{\underline{d}_{2 j}}-\frac{\left(\dot{e}-c_{2 j}\right) \dot{d}_{2 j}}{\underline{d}_{2 j}^{2}} \\
\dot{\bar{B}}_{2 j} & =\frac{\ddot{e}-\dot{c}_{2 j}}{\bar{d}_{2 j}}-\frac{\left(\dot{e}-c_{2 j}\right) \dot{\bar{d}}_{2 j}}{\bar{d}_{2 j}^{2}}
\end{aligned}
$$

Substituting Eqs. 8.1 into Eq. 4.9, and 8.3 into 4.11, respectively, and then their respective time derivatives can be written as

$$
\begin{aligned}
& \underline{\dot{\mu}}_{1 i}(e)=-\underline{\dot{A}}_{1 i} \operatorname{sgn}\left(\underline{A}_{1 i}\right) \\
& \underline{\dot{\mu}}_{2 j}(\dot{e})=-\underline{\dot{B}}_{2 j} \operatorname{sgn}\left(\underline{B}_{2 j}\right)
\end{aligned}
$$

Substituting Eqs. 4.24, 4.25 and 8.5 into Eq. 8.9 and substituting Eqs. 4.27, 4.28 and 8.7 into Eq. 8.10 , thus

$$
\underline{\dot{\mu}}_{1 i}(e)=-\eta \underline{\mu}_{1 i} \operatorname{sgn}\left(u_{p d}\right)
$$


Therefore, the time derivative of Eq. 4.18 is

$$
\underline{\dot{\mu}}_{2 j}(\dot{e})=-\eta \underline{\mu}_{2 j} \operatorname{sgn}\left(u_{p d}\right)
$$

$$
\underline{\underline{W}}_{i j}=\underline{\dot{\mu}}_{1 i} \underline{\mu}_{2 j}+\underline{\mu}_{1 i} \underline{\dot{\mu}}_{2 j}=-2 \eta \underline{W}_{i j} \operatorname{sgn}\left(u_{p d}\right)
$$

In such case, we can obtain the time derivative of the Eq. 4.21,

$$
\begin{aligned}
& \underline{\widetilde{W}}_{i j}=\frac{\underline{\underline{W}}_{i j}}{\sum_{i=1}^{I} \sum_{j=1}^{J} \underline{W}_{i j}}-\frac{\underline{W}_{i j} \sum_{i=1}^{I} \sum_{j=1}^{J} \underline{\underline{W}}_{i j}}{\left(\sum_{i=1}^{I} \sum_{j=1}^{J} \underline{W}_{i j}\right)^{2}} \\
& =\frac{-2 \eta \underline{W}_{i j} \operatorname{sgn}\left(u_{p d}\right)}{\sum_{i=1}^{I} \sum_{j=1}^{J} \underline{W}_{i j}}-\frac{\underline{W}_{i j} \sum_{i=1}^{I} \sum_{j=1}^{J}\left(-2 \eta \underline{W}_{i j} \operatorname{sgn}\left(u_{p d}\right)\right)}{\left(\sum_{i=1}^{I} \sum_{j=1}^{J} \underline{W}_{i j}\right)^{2}} \\
& =\frac{-2 \eta \operatorname{sgn}\left(u_{p d}\right) \underline{W}_{i j}}{\sum_{i=1}^{I} \sum_{j=1}^{J} \underline{W}_{i j}}-\frac{\left(-2 \eta \operatorname{sgn}\left(u_{p d}\right)\right) \underline{W}_{i j} \sum_{i=1}^{I} \sum_{j=1}^{J} \underline{W}_{i j}}{\left(\sum_{i=1}^{I} \sum_{j=1}^{J} \underline{W}_{i j}\right)^{2}} \\
& =\frac{-2 \eta \operatorname{sgn}\left(u_{p d}\right) \underline{W}_{i j}}{\sum_{i=1}^{I} \sum_{j=1}^{J} \underline{W}_{i j}}+\frac{2 \eta \operatorname{sgn}\left(u_{p d}\right) \underline{W}_{i j}}{\sum_{i=1}^{I} \sum_{j=1}^{J} \underline{W}_{i j}} \\
& =0 .
\end{aligned}
$$

Similarly, the time derivative of $\widetilde{W}_{i j}$ can also be calculated, i.e.,

$$
\dot{\bar{W}}_{i j}=0 .
$$

Since $u_{f}=q \sum_{i=1}^{I} \sum_{j=1}^{J} f_{i j} \widetilde{W}_{i j}+(1-q) \sum_{i=1}^{I} \sum_{j=1}^{J} f_{i j} \widetilde{W}_{i j}$, therefore,

$$
\begin{aligned}
& \dot{u}_{f}=\dot{q} \sum_{i=1}^{I} \sum_{j=1}^{J} f_{i j} \widetilde{\widetilde{W}}_{i j}+q \sum_{i=1}^{I} \sum_{j=1}^{J}\left(\dot{f}_{i j} \underline{\widetilde{W}}_{i j}+f_{i j} \dot{\overrightarrow{\widetilde{W}}}_{i j}\right) \\
& -\dot{q} \sum_{i=1}^{I} \sum_{j=1}^{J} f_{i j} \widetilde{\widetilde{W}}_{i j}+(1-q) \sum_{i=1}^{I} \sum_{j=1}^{J}\left(\dot{f}_{i j} \widetilde{\widetilde{W}}_{i j}+f_{i j} \dot{\overline{\widetilde{W}}}_{i j}\right) \\
& =\dot{q} \sum_{i=1}^{I} \sum_{j=1}^{J} f_{i j}\left(\widetilde{\widetilde{W}}_{i j}-\widetilde{\widetilde{W}}_{i j}\right)+\sum_{i=1}^{I} \sum_{j=1}^{J} \dot{f}_{i j}\left[q \widetilde{\widetilde{W}}_{i j}+(1-q) \widetilde{\widetilde{W}}_{i j}\right]
\end{aligned}
$$

Considering the update rules, Eqs. 4.31 and 4.30, for $q, f_{i j}$, we can get

$$
\dot{u}_{f}=-2 \beta \operatorname{sgn}\left(u_{p d}\right)
$$

In order to analyse the stability of the controller with adaptive learning rate, the following Lyapunov function is proposed.

$$
V_{c}=\frac{1}{2} u_{p d}^{2}+\frac{1}{2 \alpha}\left(\beta-\beta^{*}\right)^{2}
$$

The time derivative of the Lyapunov function 8.48 is derived as:

$$
\begin{aligned}
& \dot{V}_{c}=u_{p d} \dot{u}_{p d}+\frac{1}{\alpha} \dot{\beta}\left(\beta-\beta^{*}\right) \\
& =u_{p d}\left(\dot{u}_{f}+\dot{u}\right)+\frac{1}{\alpha} \dot{\beta}\left(\beta-\beta^{*}\right) \\
& =u_{p d}\left[-2 \beta \operatorname{sgn}\left(u_{p d}\right)+\dot{u}\right]+\frac{1}{\alpha} \dot{\beta}\left(\beta-\beta^{*}\right) \\
& =-2 \beta\left|u_{p d}\right|+u_{p d} \dot{u}+\frac{1}{\alpha} \dot{\beta}\left(\beta-\beta^{*}\right) \\
& \leq-2 \beta\left|u_{p d}\right|+B_{\dot{u}}\left|u_{p d}\right|+\frac{1}{\alpha} \dot{\beta}\left(\beta-\beta^{*}\right)
\end{aligned}
$$


Considering the adaptation law for $\beta: \dot{\beta}=\frac{1}{2} \alpha\left|u_{p d}\right|$ and meanwhile set $B_{\dot{u}}<\frac{1}{2} \beta^{*}$, thus,

$$
\begin{aligned}
& \dot{V}_{c} \leq-2 \beta\left|u_{p d}\right|+B_{\dot{u}}\left|u_{p d}\right|+\frac{1}{2}\left(\beta-\beta^{*}\right)\left|u_{p d}\right| \\
& \dot{V}_{c} \leq-\frac{3}{2} \beta\left|u_{p d}\right|+\left(B_{\dot{u}}-\frac{1}{2} \beta^{*}\right)\left|u_{p d}\right| \\
& \dot{V}_{c}<0
\end{aligned}
$$

Therefore, $u_{p d}$ converges asymptotically to zero in a finite time and consequently the system will be stable.

\subsection{Proof of Theorem 2}

In this section, we will analyse the stability of the SMC theory-based learning algorithm for the IT2FNN using triangular MFs with uncertain center. First of all, define following variables as

$$
\begin{aligned}
\underline{C}_{1 i} & =\frac{e-\underline{c}_{1 i}}{d_{1 i}}, \\
\bar{C}_{1 i} & =\frac{e-\bar{c}_{1 i}}{d_{1 i}} \\
\underline{D}_{2 j} & =\frac{\dot{e}-\underline{c}_{2 j}}{d_{2 j}} \\
\bar{D}_{2 j} & =\frac{\dot{e}-\bar{c}_{2 j}}{d_{2 j}} .
\end{aligned}
$$

Then, their corresponding time derivatives are

$$
\begin{aligned}
\dot{C}_{1 i} & =\frac{\dot{e}-\dot{c}_{1 i}}{d_{1 i}}-\frac{\left(e-\underline{c}_{1 i}\right) \dot{d}_{1 i}}{d_{1 i}^{2}} \\
\dot{C}_{1 i} & =\frac{\dot{e}-\dot{\bar{c}}_{1 i}}{d_{1 i}}-\frac{\left(e-\bar{c}_{1 i}\right) \dot{d}_{1 i}}{d_{1 i}^{2}} \\
\underline{D}_{2 j} & =\frac{\ddot{e}-\dot{\underline{c}}_{2 j}}{d_{2 j}}-\frac{\left(\dot{e}-\underline{c}_{2 j}\right) \dot{d}_{2 j}}{d_{2 j}^{2}} \\
\dot{\bar{D}}_{2 j} & =\frac{\ddot{e}-\dot{\bar{c}}_{2 j}}{d_{2 j}}-\frac{\left(\dot{e}-\bar{c}_{2 j}\right) \dot{\sigma}_{2 j}}{d_{2 j}^{2}} .
\end{aligned}
$$

Therefore, those time derivatives of Eqs. 4.13, 4.14, 4.15 and 4.16 are

$$
\begin{gathered}
\dot{\underline{\mu}}_{1 i}(e)= \begin{cases}-\dot{\bar{C}}_{1 i} \operatorname{sgn}\left(\bar{C}_{1 i}\right), & \bar{c}_{1 i}-d_{1 i}<e<\frac{\underline{c}_{1 i}+\bar{c}_{1 i}}{2} \\
-\dot{C}_{1 i} \operatorname{sgn}\left(\underline{C}_{1 i}\right), & \frac{\underline{c}_{1 i}+\bar{c}_{1 i}}{2} \leq e<\underline{c}_{1 i}+d_{1 i} \\
0, & \text { Others }\end{cases} \\
\dot{\bar{\mu}}_{1 i}(e)= \begin{cases}-\dot{C}_{1 i} \operatorname{sgn}\left(\underline{C}_{1 i}\right), & \underline{c}_{1 i}-d_{1 i}<e<\underline{c}_{1 i} \\
0, & \underline{c}_{1 i} \leq e \leq \bar{c}_{1 i} \\
-\dot{\bar{C}}_{1 i} \operatorname{sgn}\left(\bar{C}_{1 i}\right), & \bar{c}_{1 i}<e<\bar{c}_{1 i}+d_{1 i} \\
0, & \text { Others }\end{cases} \\
\dot{\mu}_{2 j}(\dot{e})= \begin{cases}-\dot{\bar{D}}_{2 j} \operatorname{sgn}\left(\bar{D}_{2 j}\right), & \bar{c}_{2 j}-d_{2 j}<\dot{e}<\frac{\underline{c}_{2 j}+\bar{c}_{2 j}}{2} \\
-\underline{\dot{D}}_{2 j} \operatorname{sgn}\left(\underline{D}_{2 j}\right), & \frac{\underline{c}_{2 j}+\bar{c}_{2 j}}{2} \leq \dot{e}<\underline{c}_{2 j}+d_{2 j} \\
0, & \text { Others }\end{cases}
\end{gathered}
$$




$$
\dot{\bar{\mu}}_{2 j}(\dot{e})= \begin{cases}-\underline{\dot{D}}_{2 j} \operatorname{sgn}\left(\underline{D}_{2 j}\right), & \underline{c}_{2 j}-d_{2 j}<\dot{e}<\underline{c}_{2 j} \\ 0, & \underline{c}_{2 j} \leq \dot{e} \leq \bar{c}_{2 j} \\ -\dot{\bar{D}}_{2 j} \operatorname{sgn}\left(\bar{D}_{2 j}\right), & \bar{c}_{2 j}<\dot{e}<\bar{c}_{2 j}+d_{2 j} \\ 0, & \text { Others }\end{cases}
$$

Substituting Eqs. 4.33 - 4.38 into Eqs. 8.29 - 8.32 , we can obtain

$$
\begin{gathered}
\dot{\mu}_{1 i}(e)=-\eta \underline{\mu}_{1 i} \operatorname{sgn}\left(u_{p d}\right) \\
\dot{\bar{\mu}}_{1 i}(e)= \begin{cases}-\eta \bar{\mu}_{1 i} \operatorname{sgn}\left(u_{p d}\right), & \underline{c}_{1 i}-d_{1 i}<e<\underline{c}_{1 i} \\
0, & \underline{c}_{1 i} \leq e \leq \bar{c}_{1 i} \\
-\eta \bar{\mu}_{1 i} \operatorname{sgn}\left(u_{p d}\right), & \bar{c}_{1 i}<e<\bar{c}_{1 i}+d_{1 i} \\
0, & \text { Others }\end{cases} \\
\dot{\bar{\mu}}_{2 j}(\dot{e})= \begin{cases}-\eta \bar{\mu}_{2 j} \operatorname{sgn}\left(u_{p d}\right), & \underline{c}_{2 j}-d_{2 j}<\dot{e}<\underline{c}_{2 j} \\
0, & \underline{c}_{2 j} \leq \dot{e} \leq \bar{c}_{2 j} \\
-\eta \bar{\mu}_{2 j} \operatorname{sgn}\left(u_{p d}\right), & \bar{c}_{2 j}<\dot{e}<\bar{c}_{2 j}+d_{2 j} \\
0, & \text { Others }\end{cases}
\end{gathered}
$$

Since

$$
\begin{gathered}
\underline{W}_{i j}=\underline{\mu}_{1 i} \underline{\mu}_{2 j}=\left\{\begin{array}{l}
\left(1-\left|\bar{C}_{1 i}\right|\right)\left(1-\left|\bar{D}_{2 j}\right|\right), \\
\left(1-\left|\underline{C}_{1 i}\right|\right)\left(1-\left|\underline{D}_{2 j}\right|\right), \\
0 .
\end{array}\right. \\
\bar{W}_{i j}=\bar{\mu}_{1 i} \bar{\mu}_{2 j}=\left\{\begin{array}{l}
\left(1-\left|\underline{C}_{1 i}\right|\right)\left(1-\left|\underline{D}_{2 j}\right|\right), \\
1, \\
\left(1-\left|\bar{C}_{1 i}\right|\right)\left(1-\left|\bar{D}_{2 j}\right|\right), \\
0 .
\end{array}\right.
\end{gathered}
$$

Next, the derivative of Eqs. 8.37 and 8.38 with respect to time can be calculated as

$$
\begin{gathered}
\dot{\underline{W}}_{i j}=\underline{\dot{\mu}}_{1 i} \underline{\mu}_{2 j}+\underline{\mu}_{1 i} \underline{\dot{\mu}}_{2 j}=-2 \eta \underline{W}_{i j} \operatorname{sgn}\left(u_{p d}\right) \\
\dot{\bar{W}}_{i j}=\dot{\bar{\mu}}_{1 i} \bar{\mu}_{2 j}+\bar{\mu}_{1 i} \dot{\bar{\mu}}_{2 j}=\left\{\begin{array}{l}
-2 \eta \bar{W}_{i j} \operatorname{sgn}\left(u_{p d}\right), \\
0, \\
-2 \eta \bar{W}_{i j} \operatorname{sgn}\left(u_{p d}\right), \\
0 .
\end{array}\right.
\end{gathered}
$$

Define

$$
\dot{\bar{M}}_{i j}=\left\{\begin{array}{l}
-2 \eta \bar{W}_{i j} \operatorname{sgn}\left(u_{p d}\right), \\
-2 \eta \operatorname{sgn}\left(u_{p d}\right), \\
-2 \eta \bar{W}_{i j} \operatorname{sgn}\left(u_{p d}\right), \\
0
\end{array}=-2 \eta \bar{W}_{i j} \operatorname{sgn}\left(u_{p d}\right)\right.
$$




$$
\dot{\bar{N}}_{i j}=\left\{\begin{array}{l}
0, \\
2 \eta \operatorname{sgn}\left(u_{p d}\right), \\
0, \\
0 .
\end{array}\right.
$$

therefore, $\dot{\bar{W}}_{i j}=\dot{\bar{M}}_{i j}+\dot{\bar{N}}_{i j}$.

In such case, we can obtain the time derivative of the Eq. 4.21,

$$
\begin{aligned}
& \dot{\vec{W}}_{i j}=\frac{\underline{W}_{i j}}{\sum_{i=1}^{I} \sum_{j=1}^{J} \underline{W}_{i j}}-\frac{\underline{W}_{i j} \sum_{i=1}^{I} \sum_{j=1}^{J} \underline{\underline{W}}_{i j}}{\left(\sum_{i=1}^{I} \sum_{j=1}^{J} \underline{W}_{i j}\right)^{2}} \\
& =\frac{-2 \eta \underline{W}_{i j} \operatorname{sgn}\left(u_{p d}\right)}{\sum_{i=1}^{I} \sum_{j=1}^{J} \underline{W}_{i j}}-\frac{\underline{W}_{i j} \sum_{i=1}^{I} \sum_{j=1}^{J}\left(-2 \eta \underline{W}_{i j} \operatorname{sgn}\left(u_{p d}\right)\right)}{\left(\sum_{i=1}^{I} \sum_{j=1}^{J} \underline{W}_{i j}\right)^{2}} \\
& =\frac{-2 \eta \operatorname{sgn}\left(u_{p d}\right) \underline{W}_{i j}}{\sum_{i=1}^{I} \sum_{j=1}^{J} \underline{W}_{i j}}-\frac{\left(-2 \eta \operatorname{sgn}\left(u_{p d}\right)\right) \underline{W}_{i j} \sum_{i=1}^{I} \sum_{j=1}^{J} \underline{W}_{i j}}{\left(\sum_{i=1}^{I} \sum_{j=1}^{J} \underline{W}_{i j}\right)^{2}} \\
& =\frac{-2 \eta \operatorname{sgn}\left(u_{p d}\right) \underline{W}_{i j}}{\sum_{i=1}^{I} \sum_{j=1}^{J} \underline{W}_{i j}}+\frac{2 \eta \operatorname{sgn}\left(u_{p d}\right) \underline{W}_{i j}}{\sum_{i=1}^{I} \sum_{j=1}^{J} \underline{W}_{i j}} \\
& =0 .
\end{aligned}
$$

Similarly, the time derivative of $\widetilde{W}_{i j}$ can also be calculated, i.e.,

$$
\begin{aligned}
& \dot{\overline{\widetilde{W}}}_{i j}=\frac{\dot{\bar{W}}_{i j}}{\sum_{i=1}^{I} \sum_{j=1}^{J} \bar{W}_{i j}}-\frac{\bar{W}_{i j} \sum_{i=1}^{I} \sum_{j=1}^{J} \dot{\bar{W}}_{i j}}{\left(\sum_{i=1}^{I} \sum_{j=1}^{J} \bar{W}_{i j}\right)^{2}} \\
& =\frac{\dot{\bar{M}}_{i j}+\dot{\bar{N}}_{i j}}{\sum_{i=1}^{I} \sum_{j=1}^{J} \bar{W}_{i j}}-\frac{\bar{W}_{i j} \sum_{i=1}^{I} \sum_{j=1}^{J}\left(\dot{\bar{M}}_{i j}+\dot{\bar{N}}_{i j}\right)}{\left(\sum_{i=1}^{I} \sum_{j=1}^{J} \bar{W}_{i j}\right)^{2}} \\
& =\frac{\dot{\bar{M}}_{i j}}{\sum_{i=1}^{I} \sum_{j=1}^{J} \bar{W}_{i j}}-\frac{\bar{W}_{i j} \sum_{i=1}^{I} \sum_{j=1}^{J} \dot{\bar{M}}_{i j}}{\left(\sum_{i=1}^{I} \sum_{j=1}^{J} \bar{W}_{i j}\right)^{2}} \\
& +\frac{\dot{\bar{N}}_{i j}}{\sum_{i=1}^{I} \sum_{j=1}^{J} \bar{W}_{i j}}-\frac{\bar{W}_{i j} \sum_{i=1}^{I} \sum_{j=1}^{J} \dot{\bar{N}}_{i j}}{\left(\sum_{i=1}^{I} \sum_{j=1}^{J} \bar{W}_{i j}\right)^{2}} \\
& =\frac{-2 \eta \operatorname{sgn}\left(u_{p d}\right) \bar{W}_{i j}}{\sum_{i=1}^{I} \sum_{j=1}^{J} \bar{W}_{i j}}-\frac{\left(-2 \eta \operatorname{sgn}\left(u_{p d}\right)\right) \bar{W}_{i j} \sum_{i=1}^{I} \sum_{j=1}^{J} \bar{W}_{i j}}{\left(\sum_{i=1}^{I} \sum_{j=1}^{J} \bar{W}_{i j}\right)^{2}} \\
& +\frac{\dot{\bar{N}}_{i j}}{\sum_{i=1}^{I} \sum_{j=1}^{J} \bar{W}_{i j}}-\frac{\bar{W}_{i j} \sum_{i=1}^{I} \sum_{j=1}^{J} \dot{\bar{N}}_{i j}}{\left(\sum_{i=1}^{I} \sum_{j=1}^{J} \bar{W}_{i j}\right)^{2}} \\
& =\frac{-2 \eta \operatorname{sgn}\left(u_{p d}\right) \bar{W}_{i j}}{\sum_{i=1}^{I} \sum_{j=1}^{J} \bar{W}_{i j}}+\frac{2 \eta \operatorname{sgn}\left(u_{p d}\right) \bar{W}_{i j}}{\sum_{i=1}^{I} \sum_{j=1}^{J} \bar{W}_{i j}} \\
& +\frac{\dot{\bar{N}}_{i j}}{\sum_{i=1}^{I} \sum_{j=1}^{J} \bar{W}_{i j}}-\frac{\bar{W}_{i j} \sum_{i=1}^{I} \sum_{j=1}^{J} \dot{\bar{N}}_{i j}}{\left(\sum_{i=1}^{I} \sum_{j=1}^{J} \bar{W}_{i j}\right)^{2}} \\
& =\frac{\dot{\bar{N}}_{i j}}{\sum_{i=1}^{I} \sum_{j=1}^{J} \bar{W}_{i j}}-\frac{\bar{W}_{i j} \sum_{i=1}^{I} \sum_{j=1}^{J} \dot{\bar{N}}_{i j}}{\left(\sum_{i=1}^{I} \sum_{j=1}^{J} \bar{W}_{i j}\right)^{2}} .
\end{aligned}
$$


Since $u_{f}=q \sum_{i=1}^{I} \sum_{j=1}^{J} \widetilde{W}_{i j} f_{i j}+(1-q) \sum_{i=1}^{I} \sum_{j=1}^{J} \widetilde{W}_{i j} f_{i j}$, therefore,

$$
\begin{aligned}
& \dot{u}_{f}=\dot{q} \sum_{i=1}^{I} \sum_{j=1}^{J} f_{i j} \widetilde{\underline{W}}_{i j}+q \sum_{i=1}^{I} \sum_{j=1}^{J}\left(\dot{f}_{i j} \widetilde{\widetilde{W}}_{i j}+f_{i j} \underline{\dot{W}}_{i j}\right) \\
& -\dot{q} \sum_{i=1}^{I} \sum_{j=1}^{J} f_{i j} \widetilde{\bar{W}}_{i j}+(1-q) \sum_{i=1}^{I} \sum_{j=1}^{J}\left(\dot{f}_{i j} \widetilde{\bar{W}}_{i j}+f_{i j} \dot{\bar{W}}_{i j}\right) \\
& =\dot{q} \sum_{i=1}^{I} \sum_{j=1}^{J} f_{i j}\left(\widetilde{\widetilde{W}}_{i j}-\widetilde{\widetilde{W}}_{i j}\right)+q \sum_{i=1}^{I} \sum_{j=1}^{J} f_{i j} \underline{\tilde{W}}_{i j}+ \\
& \sum_{i=1}^{I} \sum_{j=1}^{J} \dot{f}_{i j}\left[q \widetilde{\widetilde{W}}_{i j}+(1-q) \widetilde{\bar{W}}_{i j}\right]+(1-q) \sum_{i=1}^{I} \sum_{j=1}^{J} f_{i j} \dot{\overline{\bar{W}}}_{i j} \\
& =\dot{q} \sum_{i=1}^{I} \sum_{j=1}^{J} f_{i j}\left(\widetilde{\widetilde{W}}_{i j}-\widetilde{\bar{W}}_{i j}\right)+\sum_{i=1}^{I} \sum_{j=1}^{J} \dot{f}_{i j}\left[q \underline{\widetilde{W}}_{i j}+(1-q) \widetilde{\bar{W}}_{i j}\right] \\
& +(1-q) \sum_{i=1}^{I} \sum_{j=1}^{J} f_{i j} \dot{\bar{W}}_{i j}
\end{aligned}
$$

Considering the update rules for $q, f_{i j}$, i.e., Eq. 4.40 and 4.39 , we can get

$$
\begin{gathered}
\dot{u}_{f}=-2 \eta \operatorname{sgn}\left(u_{p d}\right)+(1-q) \frac{\sum_{i=1}^{I} \sum_{j=1}^{J} f_{i j} \dot{\bar{N}}_{i j}}{\sum_{i=1}^{I} \sum_{j=1}^{J} \bar{W}_{i j}} \\
-(1-q) \frac{\sum_{i=1}^{I} \sum_{j=1}^{J}\left(f_{i j} \bar{W}_{i j} \sum_{i=1}^{I} \sum_{j=1}^{J} \dot{\bar{N}}_{i j}\right)}{\left(\sum_{i=1}^{I} \sum_{j=1}^{J} \bar{W}_{i j}\right)^{2}} .
\end{gathered}
$$

Define

$$
\begin{aligned}
D & =(1-q) \frac{\sum_{i=1}^{I} \sum_{j=1}^{J} f_{i j} \dot{\bar{N}}_{i j}}{\sum_{i=1}^{I} \sum_{j=1}^{J} \bar{W}_{i j}} \\
& -(1-q) \frac{\sum_{i=1}^{I} \sum_{j=1}^{J}\left(f_{i j} \bar{W}_{i j} \sum_{i=1}^{I} \sum_{j=1}^{J} \dot{\bar{N}}_{i j}\right)}{\left(\sum_{i=1}^{I} \sum_{j=1}^{J} \bar{W}_{i j}\right)^{2}} .
\end{aligned}
$$

Since $|q|<1,\left|f_{i j}\right|<B_{f},\left|\bar{W}_{i j}\right| \leq 1,\left|\dot{\bar{N}}_{i j}\right|<2 \eta$, thus, $D$ is bounded and its maximum value is $D_{m}$.

In order to analyse the stability of the controller with adaptive learning rate, the following Lyapunov function is proposed.

$$
V_{c}=\frac{1}{2} u_{p d}^{2}+\frac{1}{2 \alpha}\left(\beta-\beta^{*}\right)^{2}
$$

The time derivative of the Lyapunov function 8.48 is derived as:

$$
\begin{aligned}
& \dot{V}_{c}=u_{p d} \dot{u}_{p d}+\frac{1}{\alpha} \dot{\beta}\left(\beta-\beta^{*}\right)=u_{p d}\left(\dot{u}_{f}+\dot{u}\right)+\frac{1}{\alpha} \dot{\beta}\left(\beta-\beta^{*}\right) \\
& =u_{p d}\left[-2 \beta \operatorname{sgn}\left(u_{p d}\right)+D+\dot{u}\right]+\frac{1}{\alpha} \dot{\beta}\left(\beta-\beta^{*}\right) \\
& =-2 \beta\left|u_{p d}\right|+u_{p d}(D+\dot{u})+\frac{1}{\alpha} \dot{\beta}\left(\beta-\beta^{*}\right) \\
& \leq-2 \beta\left|u_{p d}\right|+\left(D_{m}+B_{\dot{u}}\right)\left|u_{p d}\right|+\frac{1}{\alpha} \dot{\beta}\left(\beta-\beta^{*}\right)
\end{aligned}
$$


Considering the adaptation law for $\beta: \dot{\beta}=\frac{1}{2} \alpha\left|u_{p d}\right|$ and meanwhile set $D_{m}+B_{\dot{u}}<\frac{1}{2} \beta^{*}$, i.e., $D_{m}+B_{\dot{u}}-$ $\frac{1}{2} \beta^{*}<0$ thus,

$$
\begin{aligned}
& \dot{V}_{c} \leq-2 \beta\left|u_{p d}\right|+\left(D_{m}+B_{\dot{u}}\right)\left|u_{p d}\right|+\frac{1}{2}\left(\beta-\beta^{*}\right)\left|u_{p d}\right| \\
& \dot{V}_{c} \leq-\frac{3}{2} \beta\left|u_{p d}\right|+\left(D_{m}+B_{\dot{u}}-\frac{1}{2} \beta^{*}\right)\left|u_{p d}\right| \\
& \dot{V}_{c}<0
\end{aligned}
$$

Therefore, $u_{p d}$ converges asymptotically to zero in a finite time and consequently the system will be stable.

\subsection{Proof of Theorem 3}

Consider a second order system as

$$
\begin{aligned}
& \dot{x}_{1}=x_{2} \\
& \dot{x}_{2}=f\left(x_{1}, x_{2}\right)+u .
\end{aligned}
$$

As mentioned before, both $e$ and $\dot{e}$ are error signal and its time derivative. Here, $e=x_{r}-x_{1}, \dot{e}=\dot{x}_{r}-\dot{x}_{1}$, then $\ddot{e}=\ddot{x}_{r}-\dot{x}_{2}=\ddot{x}_{r}-f\left(x_{1}, x_{2}\right)-u$.

For a candidate Lyapunov function

$$
V_{a}=\frac{1}{2} K_{p} e^{2}+\frac{1}{2} \dot{e}^{2}+\frac{1}{2 \gamma}\left(K_{a}-K_{a}^{*}\right)^{2}, \gamma>0
$$

its time derivative is

$$
\begin{aligned}
\dot{V}_{a} & =K_{p} e \dot{e}+\dot{e} \ddot{e}+\frac{1}{\gamma} \dot{K}_{a}\left(K_{a}-K_{a}^{*}\right), \gamma>0 \\
& =K_{p} e \dot{e}+\dot{e}\left(\ddot{x}_{r}-f\left(x_{1}, x_{2}\right)-u\right)+\frac{1}{\gamma} \dot{K}_{a}\left(K_{a}-K_{a}^{*}\right)
\end{aligned}
$$

The control signal $u$ consists of three parts: an output of the PD controller, $u_{p d}=K_{p} e+K_{d} \dot{e}$, the output of the T2FNN, $u_{f}$ and the robustness term, $u_{a}=K_{a} \operatorname{sgn}(\dot{e})+\ddot{x}_{r}$, where $\dot{K}_{a}=\lambda|\dot{e}|, \lambda>0$,

$$
\begin{aligned}
u & =u_{p d}+u_{a}-u_{f} \\
& =K_{p} e+K_{d} \dot{e}+K_{a} \operatorname{sgn}(\dot{e})+\ddot{x}_{r}-u_{f}
\end{aligned}
$$

Considering that $\left|f\left(x_{1}, x_{2}\right)\right|<B_{f 1},\left|u_{f}\right|<B_{u f}$ and meanwhile satisfying that $B_{f 1}+B_{u f}<K_{a}^{*}$, therefore,

$$
\begin{aligned}
\dot{V}_{a} & =K_{p} e \dot{e}+\dot{e} \ddot{e}+\frac{1}{\gamma} \dot{K}_{a}\left(K_{a}-K_{a}^{*}\right), \gamma>0 \\
& =K_{p} e \dot{e}+\dot{e}\left(\ddot{x}_{r}-f\left(x_{1}, x_{2}\right)-K_{p} e-K_{d} \dot{e}-K_{a} \operatorname{sgn}(\dot{e})-\ddot{x}_{r}+u_{f}\right) \\
& +\frac{1}{\gamma} \dot{K}_{a}\left(K_{a}-K_{a}^{*}\right) \\
& =-K_{d} \dot{e}^{2}-K_{a}|\dot{e}|+\dot{e}\left(-f\left(x_{1}, x_{2}\right)+u_{f}\right)+\left(K_{a}-K_{a}^{*}\right)|\dot{e}| \\
& =-K_{d} \dot{e}^{2}+\dot{e}\left(-f\left(x_{1}, x_{2}\right)+u_{f}\right)-K_{a}^{*}|\dot{e}| \\
& <-K_{d} \dot{e}^{2}+\left(B_{f 1}+B_{u f}-K_{a}^{*}\right)|\dot{e}| \\
& <0
\end{aligned}
$$

This means that the error signal will finally converge to zero and the whole system will keep stable. The proof process is on the basis of the work in (165). For more detail information, readers can refer to (165). 